\title{
Loss Mechanisms in Non-Fullerene Organic Solar Cells
}

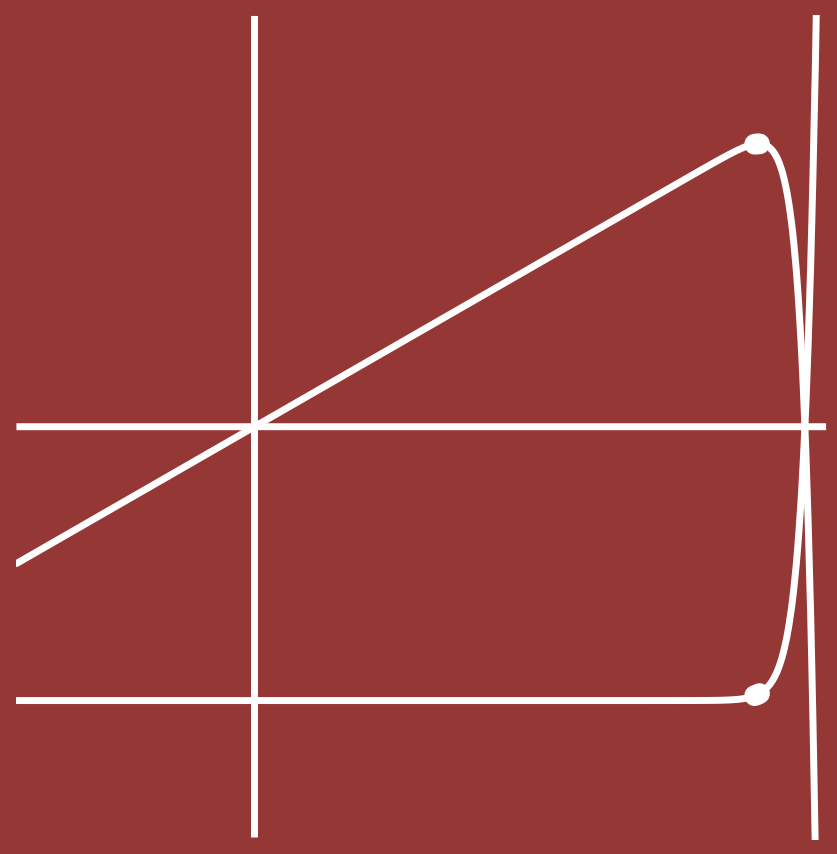

Huotian Zhang

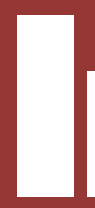





\title{
Loss Mechanisms in Non-Fullerene Organic Solar Cells
}

\author{
Huotian Zhang
}


During the course of research underlying this thesis, Huotian Zhang was enrolled in the graduate school Agora Materiae, a multidisciplinary doctoral program at Linköping University, Sweden.

This work is licensed under a Creative Commons "Attribution-NonCommercial 4.0 International" license.

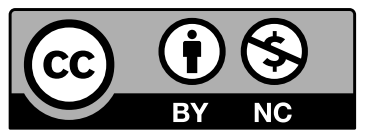

Loss Mechanisms in Non-Fullerene Organic Solar Cells

(C) Huotian Zhang, 2021

ISBN 978-91-7929-034-4

ISSN 0345-7524

Printed by LiU-Tryck, Linköping, Sweden, 2021 
Photovoltaics are one of the most important sustainable energy sources in the 21st century. Among photovoltaics, organic solar cells (OSCs) offer many advantages such as the ease of processing, light weight, the potential for flexible devices, and tunable properties. Their unique nature and complexity present a fascinating charm, attracting many researchers. Thanks to researchers' efforts, the power conversion efficiency (PCE) of OSCs has been boosted from below $1 \%$ to $19 \%$ over the last three decades. Despite the exciting PCE, some problems remain unsolved, for example, the large voltage loss and unsatisfying long-term stability. This thesis aims to understand the fundamental physics of the state-of-the-art OSCs, especially the loss mechanisms. Ultimately, properly understanding the mechanisms will serve as the basis of OSC's further improvements and commercialization.

The beginning of this thesis introduces basic concepts about semiconductor physics and donor-acceptor OSCs, explaining the generation of electricity from light and the fundamentals of organic electronics. Subsequently, the detailed balance in a solar cell is reviewed, which is the basis of voltage loss analysis. In this section, we see how the generation, recombination, and output of charges reach a balance. Then, the way to determine the voltage loss is shown, and the most recent understandings in reducing this loss are reviewed. The fill factor, as a measure of the quality of a solar cell, is a complex parameter, especially in OSCs. The latter part of this thesis starts from the photophysical processes in an OSC, and then relates intrinsic parameters to the fill factor. Figure of merits have been employed to express the fill factor analytically. Finally, experimental methods and basic principles for the previous analysis are introduced, including Fourier transform infrared spectroscopy, external quantum efficiency of photovoltaics, spectrograph for electroluminescence or photoluminescence, transient absorption, and time-delayed collection field. Overall, this thesis combines thermal dynamics and charge dynamics to analyse voltage losses and fill factor losses. The author hopes this work can contribute to a better understanding of the loss mechanisms in OSCs.

Keywords: photovoltaics, organic solar cells, non-fullerene, charge recombination, voltage loss, fill factor 


\section{POPULÄRVETENSKAPLIG SAMMANFATTNING}

Solceller är ett av 2000-talets viktigaste hållbara energikällor. Bland solceller erbjuder organiska solceller (OSC) många fördelar som enkel bearbetning, lättvikt, potential för flexibilitet och avstämbara egenskaper. Dess märkliga natur och komplexitet utgör en fascinerande lockelse som attraherar många forskare. Tack vare forskarnas ansträngningar har OSC:ernas verkningsgrad ökat från 1\% till 19\% under de senaste tre decennierna. Trots den spännande verkningsgraden återstår vissa problem att lösa, till exempel den stora spänningsförlusten och den långsiktiga stabiliteten. Denna avhandling syftar till att förstå den grundläggande fysiken hos de senaste OSC:erna, särskilt förlustmekanismen. I slutändan kommer en korrekt förståelse av mekanismerna att bli en arbetsgrund för OSC:ers ytterligare förbättring och kommersialisering.

Detta arbete fokuserar på förlustmekanismerna för OSC, särskilt tomgångspänningen och fyllningsfaktorn. I början av denna avhandling introduceras grundläggande begrepp om halvledarfysik och donator-acceptor-OSC:er. Denna del förklarar elproduktionen från ljus och grunderna i organisk elektronik. Därefter ses den detaljerade balansen i en solcell över, vilket är grunden för spänningsförlustanalysen. I den här delen ser vi hur ingångsparameterar, rekombination och utgångsparametrar hänger samman. Sedan visas sättet att bestämma spänningsförlusten, och de senaste rönen för att minska förlusten ses över.

Fyllningsfaktorn, som ett mått på en solcells kvalitet, är en komplex parameter, särskilt i OSC:er. Den senare delen av denna avhandling utgår från de fotofysiska processerna i en OSC och relaterar sedan inneboende parametrar till fyllningsfaktorn. Godhetstal har använts för att uttrycka fyllningsfaktorn analytiskt. I slutändan introduceras experimentella metoder och grundläggande principer för den tidigare analysen, inklusive Fourier transform infraröd spektroskopi, extern kvanteffektivitet hos solceller, spektrografi för elektroluminescens eller fotoluminiscens, övergående-absorption och tidsfördröjd insamlingsfält. Sammantaget kombinerade avhandlingen termisk-dynamik och laddningsdynamik för att analysera spänningsförluster och fyllningsfaktorförluster. Författaren hoppas att detta arbete kan bidra till en bättre förståelse av förlustmekanismerna i OSC. 


\section{Acknowledgments}

This thesis would not have been possible without the support of so many people, during both work and my personal life, through the whole four years of my doctoral study. It is my great pleasure to express my sincere gratitude to all the people for inspiring and helping me.

First and foremost, I would like to express my deepest gratitude to Feng Gao for supervising me. Thank you for providing freedom and support on my research path. I can enjoy exploring science to the maximum extent possible at my own preference and pace, with abundant resources inside and outside the group, meanwhile without pressure for failure. I can always learn and get inspired by regular discussions with you, not only in terms of fundamental knowledge and experimental techniques, but also in terms of critical thinking, scientific vision, team leadership and life management. It is you that made everything possible and achievable.

I am also grateful to my co-supervisor, Olle Inganäs and Weimin Chen, for your great support and guidance. Especially for Olle, thank you for so many stimulating questions and constructive comments, whenever we had academic seminars or personal discussions.

I owe my thanks to my wonderful collaborators, without whom this work would not have been possible. Lorena Perdigón-Toro, thank you for introducing me to your lab and for accompanying me during all the experiments at Potsdam. I enjoyed working and discussing with you. I always got inspired by your thoughts and got encouraged by your dedication. Rokas Jasiūnas, thank you for helping me with the transient measurements and analysis, and for stimulating discussions. I also enjoyed the time doing experiments with you. I would like to thank Xiane Li, Chuanfei Wang, and Qilun Zhang for UPS characterization, data analysis, and fruitful discussions. Yunhao Cai, thank you for providing the materials and the device fabrication skills. Wenchao Huang, thank you for measuring GIWAXS and analyzing the data. I want to take the opportunity to thank all my collaborators: Shuixing Li, Lingling Zhan, Hao-Wen Cheng, Pei Chen, Jeromy J Rech, Tanvi Upreti, Can Zhu, Fan Wu, Guodong Xu, Dan He.

I would like to acknowledge Martijn Kemerink, Mats Fahlman, Dieter Neher, Martin Stolterfoht, Safa Shoaee, Arkady Yartsev, Thomas Kirchartz, Vidmantas Gulbinas, Nir Tessler, Fengling Zhang, Yanming Sun and Yongfang Li for those highly useful, inspir- 
ing discussions and advices on my research, and showing me the beauty of different research fields.

Additional thanks go to Feng Gao, Deping Qian, Li Wan, Nakul Jain, Jiajun Qin, David Hardy, Rui Shu, Yonghong Wang, Xinyi Cai, Bei Yang, Jun Yuan, Rui Zhang, and Jie Ren, who helped revise my thesis.

I would like to thank all the former and current members of OPV team in the GAO group. All of you have been my great friends and colleagues. Deping Qian and Yuming Wang, thank you so much for leading me to the lab, teaching me most of the experimental techniques, and giving me a lot of support in the research. I owe many thanks to Jianwei Yu, for sharing your knowledge in materials synthesis, selfless help in the lab, ordering consumables, and providing me materials. A special thank you to Jun Yuan, for providing the state-of-the-art materials, inspiring discussions, and generous help, whose dedication has always been an encouragement to me. I need to thank Rui Zhang a lot, for sharing extensive insights in condensed matter physics, providing experimental help, and discussing the future. I would like to thank Xuehong Zhou, for providing experimental testing and theoretical modeling on transient absorption, as well as knowledge in photophysics. I owe my thanks to Bei Yang, for sharing knowledge in chemistry, skills in experiments, and experience about life. I want to thank Nakul Jain, for taking care of the OPV lab, providing superb supports in instruments, and exciting discussions. Many thanks to Shan Anjum, for helping me translate "popular scientific summary" into Swedish and revise the abstract. I also need to thank Jie Ren, for teaching me fabricating, characterizing, and analysing organic transistors. Thanks to Fuwen Zhao, for providing me experimental help.

I would like to thank the former and current colleagues at Biomolecular and Organic Electronics (Biorgel), and now also Electronic and Photonic materials (EFM): Feng Wang, Weidong Xu, Jie Yang, Weidong Cai, Naresh Kumar Kumawat, Mengyun Chen, Yuequn Shang, Zhan Chen,Xiyu Luo, Shanpeng Wang, David Hardy, Julia Morat, Zhiqi Li, Jibin Zhang, Xin Wang, Tao Zhang, Jia Zhang, Shaorong Huang, Gulzada Beket, Xiang Xu, Guanhaojie Zheng, Mei Yang, Yan Xu, Jian Qing, Zhenyu Qi, Ziyue Yi, Yatao Zou, Haifeng Zhao, Pengpeng Teng, Hongting Chen, Min Zhang, Yusheng Yuan, Leiqiang Qin, Qingzhen Bian, Yuxin Xia, Wanzhu Cai, Ke Zhou, Xiaofeng Xu, Jie Luo, Xiaolin Zhang, Wenfei Shen, Luis Ever Aguirre, and Jiayan Cong, for practical discussion and advices on improving my experiments and collaborations. I want to say thank you to Sai Bai for hosting me when I was homeless and giving me support in both research and career; to Chunyang Yin and Bin Zhang for teaching me optical experiments; to Chunxiong Bao and Zhibo Yan for guiding me in LabVIEW programming and sharing characterization experience; to Zhongcheng Yuan, Tiankai Zhang, Weihua Ning, and Hongwei Lei for providing me devices; to Yu Wang and Muyi Zhang for support in the lab and life; to Xinyi Cai for sharing quantum chemistry knowledge and providing various support; to Fuxiang Ji and Chaoyang Kuang for helping each other along the way of PhD study; to Hongling Yu, Heyong Wang, and Xiaoke Liu for sharing the experience of $\mathrm{PhD}$ studies or lab management and the understandings of experiments; to Jiajun Qin, Yong Wang, Li Wan, Tianjun Liu, Fanglong Yuan, and Yonghong Wang, for sharing the knowledge of science and the experience of career development; to Yanfeng Liu, Yingzhi Jin, Nannan Yao, Lianlian Liu, and Lei Wang for sharing information of research, life and PhD defense; to Linlong Deng and Junjie Xie for helping 
me in in my tough time; to Carlito Ponseca for teaching me transient spectroscopy and sharing experience in Terahertz experiments; to Max Karlsson and Greta Elovsson for ordering many experimental equipments; to Ann-Charlotte Svensson Holm and Wendela Yonar for administrative support; to Niclas Solin for providing support in lab management and administration as the division head.

I had a very nice time being in charge of the darkroom for optical and electrical characterization. I owe my thanks to those colleagues who are willing to be involved in the management and maintenance in the darkroom. You have made experiments easier for everyone.

Many thanks to my friends and colleagues from the Agora Materiae graduate school, the Agora Student Council, and the former and current heads of Agora, Per-Olof Holz, Fredrik Karlsson and Caroline Brommesson, for all the nice study trips, seminars, summer conferences and discussions.

I had also a very nice time being a member of the PhD Reference Group (now Doctoral Student Council - DoStuC) at IFM. I owe my thanks to the colleagues in the group for accepting me, guiding me, and encouraging me. I was very pleased to be involved in many interesting projects and very honored to work with you guys.

I would like to thank Rickard Liljedahl for helping me make quote inquiries and for making purchase orders. Many thanks to Sven Andersson for helping me with technical issues. I want to thank all the members of the former Equal Opportunities Group, especially Galia Pozina, for efficiently solving the issues raised by me as a representative of the PhD Reference Group. My sincere thanks go to Chunxia Du, for always listening with patience, encouraging and helping me.

I would like to thank my friends at IFM and in Sweden: Binbin Xin, Xin Zhang, Zhixing Wu, Hengfang Zhang, Yuqing Huang, Shula Chen, Jiwen Hu, Yuchen Shi, Pimin Zhang, Jinhao Xu, Dunyong Deng, Guangzheng Zuo, Qingqing Wang, Junda Huang, Shaobo Han, Canyan Che, Shangzhi Chen, Fei Zhao, Sijian Yuan, Kunlong Yang, Xianshao Zou, Mao Wang, Chen Luo, Claudia Schnitter, Marius Rodner, Pernilla Helmer, Sachin Sharma, and Jui-Che Chang. I really enjoyed the time we spend together. Special thanks go to Rui Shu, Quanzheng Tao, Jie Zhou, Lingyin Meng, Danfeng Cao, Chi Xiao, Panjie Yan, and Ming Guo for sharing experiences in research and life.

I feel grateful to China Scholarship Council for fully funding my four-year PhD study. I also thank Yiqiang Zhan, my former supervisor, who gives me much guidance in both research and life during my master study.

And finally, I would like to express my deepest gratefulness to my family members, whose unconditional support allows me to devote myself to what I love. My sincere thanks go to my wife Rong Qu for encouraging and supporting me along the way. The last bit goes to our lovely daughter Xiaorong. Lucky to meet you.

PS: After writing this, I found that much more people helped me than I initially thought. I tried not to miss any of them, but there might be some more. I hope these people who made me feel kind and warm during my PhD study can also be treated tenderly by life.

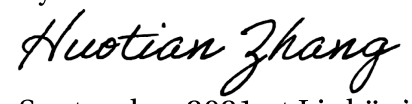

September 2021 at Linköping 

Abstract $\quad$ i

Acknowledgments $\quad$ iii

1 Introduction 1

1.1 Climate change and energy supply . . . . . . . . . . . . 1

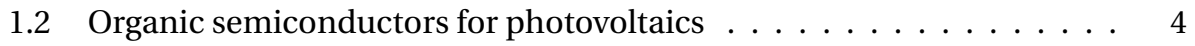

1.3 Aims and structure of this thesis ............. 5

2 Basic concepts $\quad 7$

2.1 The photovoltaic effect ................. 7

2.1.1 From photonic energy to chemical potential . . . . . . . . 7

2.1.2 From chemical potential to electrical energy . . . . . . . . . . 9

2.1.3 Characteristics of solar cells . . . . . . . . . . . . . . . 12

2.2 Donor-acceptor organic solar cells . . . . . . . . . . . . . . . . 14

2.2.1 Energetics of organic semiconductors . . . . . . . . . . . 14

2.2.2 Interfacial charge-transfer states $\ldots \ldots \ldots 17$

3 Thermal dynamics and the voltage loss 19

3.1 Detailed balance . . . . . . . . . . . . . . . . . . 19

3.1.1 Solar spectrum and the black-body radiation . . . . . . . . . 19

3.1.2 Shockley-Queisser limit . . . . . . . . . . . . . . . 21

3.1.3 Reciprocity between absorption and emission . . . . . . . . 22

3.2 Voltage losses in single-junction solar cells . . . . . . . . . . . . . 24

3.2.1 Defining an optical gap . . . . . . . . . . . . . . 24

3.2.2 Quantifying voltage losses . . . . . . . . . . . . . . 26

3.3 Reducing voltage losses in organics solar cells . . . . . . . . . . . . 27

3.3 .1 Energy gap law . . . . . . . . . . . . . . . . 28

3.3.2 Energetic offsets . . . . . . . . . . . . . . 29 
3.3.3 Other methods to reduce non-radiative losses . . . . . . . . 30

4 Charge dynamics and the fill factor 33

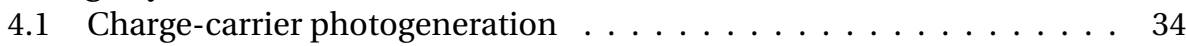

4.1.1 Photon absorption and exciton generation . . . . . . . . 34

4.1 .2 Exciton diffusion . . . . . . . . . . . . . . . 34

4.1 .3 Charge transfer . . . . . . . . . . . . . . . 34

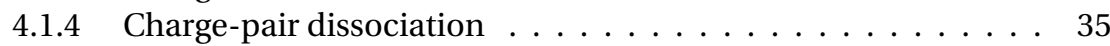

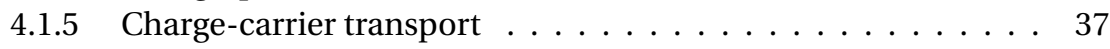

4.2 Geminate and non-geminate recombination . . . . . . . . . . . 39

4.2.1 Recombination of geminate pairs . . . . . . . . . . 39

4.2 .2 Recombination of free carriers . . . . . . . . . . . . . . . . . . . . . . . .

4.3 The fill factor . . . . . . . . . . . . . . . . . 41

4.3.1 Analytical expressions . . . . . . . . . . . . 41

4.3.2 Figure of merits for organic solar cells . . . . . . . . . . . 42

4.3.3 Development of the fill factor in organic solar cells . . . . . . 46

5 Summary and Outlook $\quad 49$

6 Methods 51

6.1 Sensitive-external-quantum-efficiency characterisation . . . . . . 51

6.1.1 Monochromatic technique . . . . . . . . . . . 51

6.1 .2 Fourier-transform technique . . . . . . . . . . 53

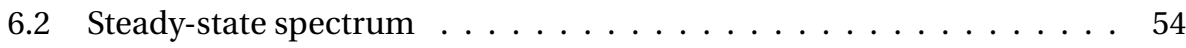

6.2 .1 Spectrograph . . . . . . . . . . . . . . . 54

6.2 .2 UV-VIS-NIR absorption . . . . . . . . . . . . . 55

6.2.3 Photoluminescence and quantum yield measurement . . . . . 55

6.2.4 External quantum efficiency for electroluminescence . . . . . . 56

6.3 Time-resolved measurements . . . . . . . . . . . . . . 57

6.3.1 Transient absorption spectroscopy . . . . . . . . . . . . 57

6.3.2 Time-delayed collection field . . . . . . . . . . . . 59

7 Results and included pappers $\quad 61$

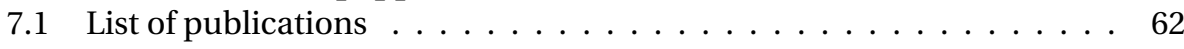

7.2 Summary of included papers . . . . . . . . . . . . . 67

$\begin{array}{ll}\text { References } & 71\end{array}$

$\begin{array}{lc}\text { Paper I } & 83\end{array}$

$\begin{array}{lr}\text { Paper II } & 121\end{array}$

$\begin{array}{ll}\text { Paper III } & 141\end{array}$

$\begin{array}{ll}\text { Paper IV } & 159\end{array}$ 


\section{CHAPTER 1}

Introduction

This chapter presents the background information and motivation for this work. Organic solar cells are widely studied but still rarely used in daily life. There is no doubt that society supports this technology and expects its potential application as renewable energy. However, the role that organic solar cells will play in the future remains unclear. Before answering this question, let us review the history and current state of the field. In the last section of the chapter, the aim and the structure of this thesis will be described.

\subsection{Climate change and energy supply}

Since the early 2000s, scientists have observed obvious climate changes [2-4]. Global warming, as one of the most significant changes, are most likely driven by human activities [5, 6]. According to the data from NASA [1], global surface average temperature has increased by over 1 degree Celsius since the pre-industry period ( Figure 1.1). At the same time, extreme weather events are becoming more serious and more frequent. From heatwaves in Sweden[7], droughts in South Africa[8], flooding in Bangladesh[9] to hurricanes in the Caribbean[10] and arctic outbreak in Texas[11], people's daily lives are becoming increasingly disrupted. Mounting evidence is showing the terrible results of global warming, drawing increasing global attention, meanwhile, the warming is accelerating faster than we thought [12].

Global warming is primarily caused by fossil fuel combustion, which increases the level of heat-absorbing greenhouse gases in the earth's atmosphere. The large-scale use of fossil fuels contributed to the Industrial Revolution. Now, fossil fuels have become the cornerstone of modern society due to their importance in transportation, power generation and industrial production. As of 2018, the world's major energy sources include oil (34\%), coal (27\%), and natural gas (24\%), with the share of fossil 

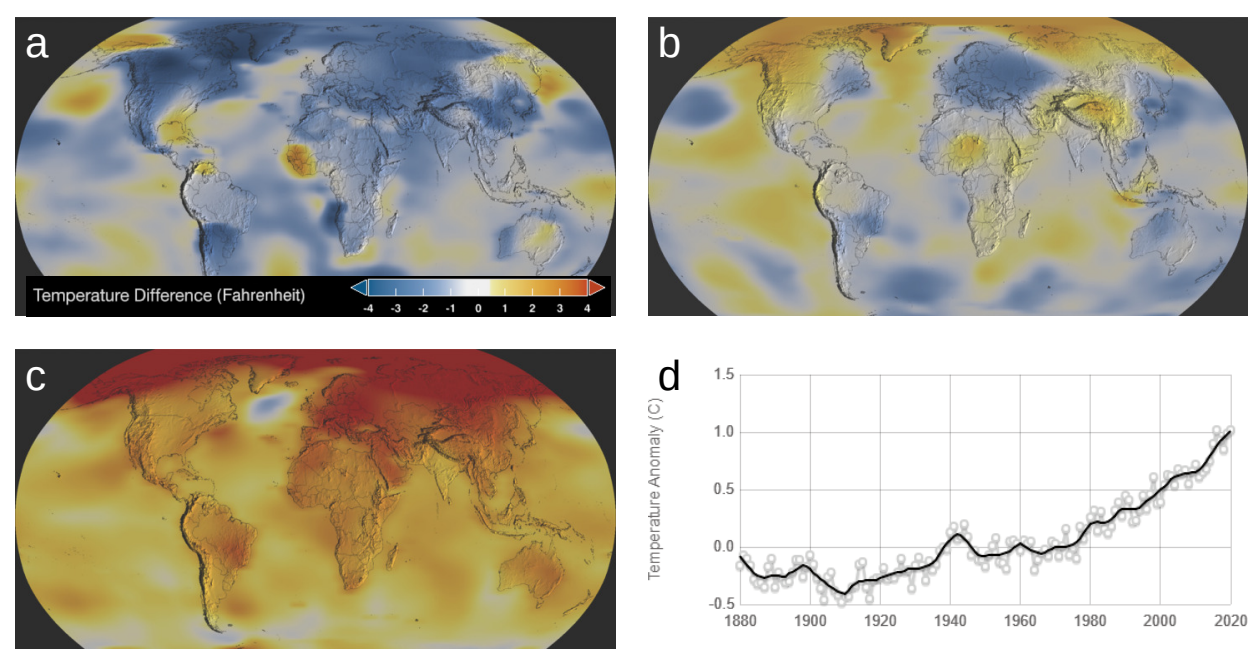

Figure 1.1 | Global warming. These graphs illustrate the global surface temperature difference in year (a) 1884, (b) 1943, and (c) 2020. d, The average temperature has increased significantly since 1880.

Data source: NASA's Goddard Institute for Space Studies (GISS) and NOAA's National Centers for Environmental Information NCEI[1]. Credit: NASA/GISS, NOAA/NCEI

fuels in the world's primary energy consumption at $85 \%$ [13]. Correspondingly, the combustion of fossil fuels produces around 35 billion tons of carbon dioxide $\left(\mathrm{CO}_{2}\right)$ per year, far exceeding the amount that can be absorbed by natural processes. As a result, there is a net increase of billion tons of $\left(\mathrm{CO}_{2}\right)$ per year [14], which leads to global warming and ocean acidification.

To stop further climate deterioration, the term "Carbon neutrality" is proposed, which refers to achieving net-zero carbon dioxide emissions. One way to implement carbon neutrality is by using renewable energy such as hydro, wind, geothermal, and solar power. According to the electricity generation amount by each energy source from 1990-2018 (Figure 1.2 a), fossil fuels still dominate the power generation despite significant increase of renewable energy sources. Hence, there are still great potentials for renewable energy sources. The solar photovoltaic (PV), as one of the promising methods for clean energy, has surged during last three decades. As shown in Figure 1.2 b, the share of solar PV in both all energy sources and renewable energy sources has increased by over three orders of magnitude from 1990 to 2018, while the total electricity consumption has kept growing. Of course, this initial rapid growth of PV benefits greatly from the support of various countries and regions. Certainly, it is attributed to the distinctive features of PV, for example, no pollution and no emission during operation, no fuel filler required, low safety risk, and great abundance of raw materials. 

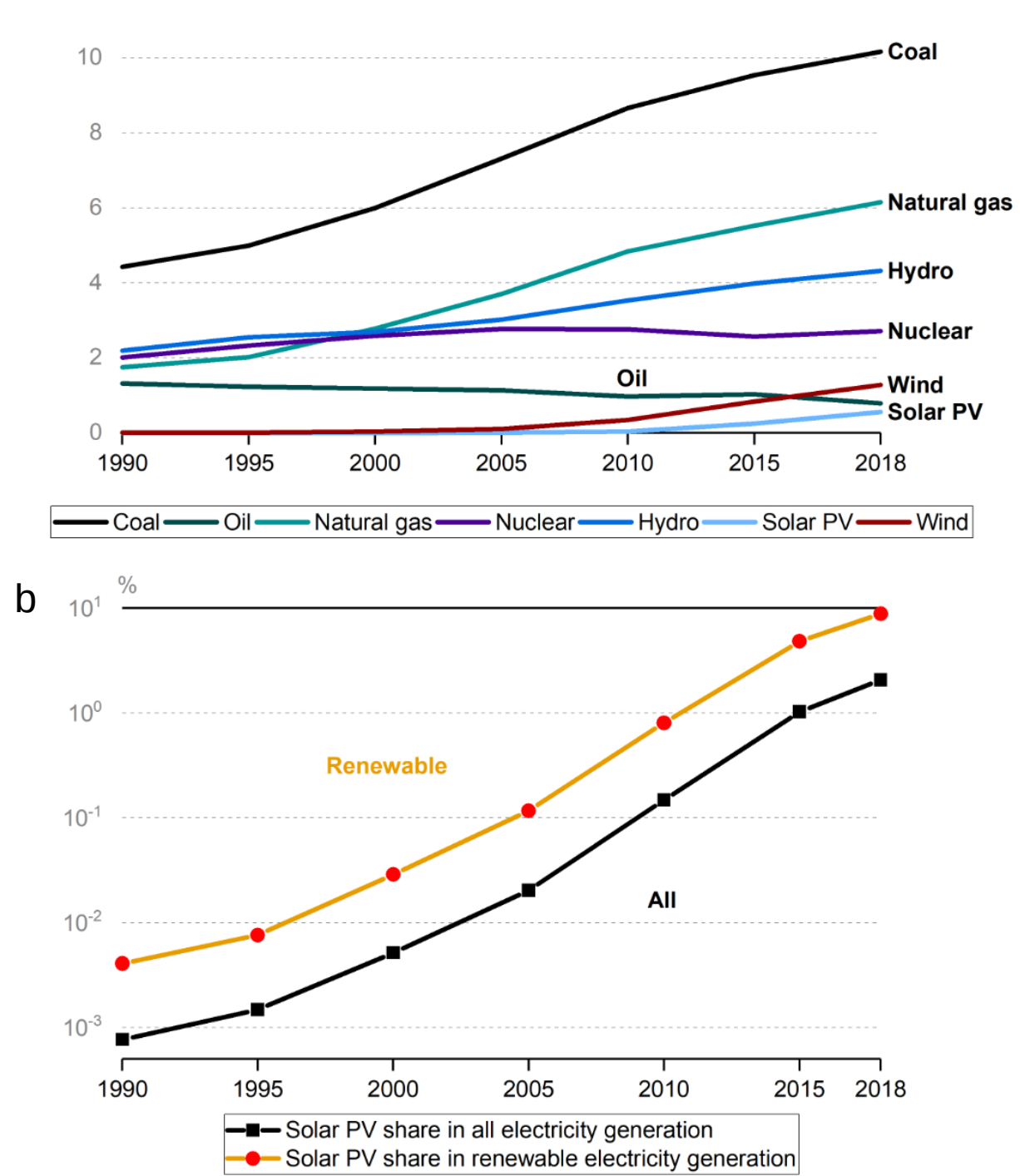

Figure 1.2 | Energy supply around the world. a, Electricity generation by source, World 1990-2018. b, The electricity generation share of solar PV in all sources and renewable sources, World 1990-2018

Data source: International Energy Agency (IEA). 


\subsection{Organic semiconductors for photovoltaics}

The term organic semiconductor implies (1) that the compounds generally contain carbon-dydrogen bonds, possibly with a few heteroatoms such as oxygen, nitrogen and sulfur, and (2) that they exhibit features typically possessed by semiconductor materials [15]. Organic semiconductors are interesting because they have the potential to combine electrical properties of semiconducting materials with chemical and mechanical advantages of organic compounds.

A semiconductor material has an electrical conductivity value falling in between that of a conductor and an insulator. Its conductive properties may be altered by introducing impurities, illumination or by external electric field. Since the middle of 20th century, the information era and the mobile Internet age have arrived one after another with the spread of personal computers and smartphones. At the same time, the semiconductor industry, which is the backbone of electronic products, has experienced booming development. Semiconductor materials have also been widely studied and each of them has its distinctive properties. For example, the high abundance, the good mobilities and the perfect $\mathrm{Si}-\mathrm{SiO}_{2}$ interface make silicon a natural pilla material for general purpose devices. The high charge-carrier mobilities, relatively insensitive to overheating and low noise of gallium arsenide make it perfect for high frequencies application such as radio circuits for 5G. Silicon carbide is widely used as power electronic devices in electric systems and high speed rails due to its high voltage and high temperature resistance.

Daily encountered organics can hardly be related to conductivity or electronics. However, this stereotypical impression has been changed when conjugated polymers with metallic transport came into the scientific spotlight around 1970s [16]. In addition, by manipulating the chemical synthesis, researchers succeeded in making organics work as semiconductors. Based on their semiconducting properties, organic semiconductors have been applied in devices such as photovoltaics (PVs), light-emitting diodes (LEDs), or field-effect transistors (FETs) [17]. However, although the organics show semiconducting properties, they have some intrinsic differences when compared with inorganic semiconductors. Organics typically consist of molecules. The intermolecular forces are widely present in organic matters, giving rise to their characteristics of softness and ease of processing. However, these also bring about localized electronic wave functions and weak dielectric screening. When electrons are excited in semiconductors, they can form a bound state with holes, which is called 'exciton'. According to the degree of charge binding or electric field screening, excitons can be divided into Wannier-Mott excitons or Frenkel excitons, respectively. In typical inorganic semiconductors, excitons are weakly bound Wannier-Mott excitons with large radii and small binding energy, which can directly separate at room temperature. In organic semiconductors, excitons are usually strongly bound Frenkel excitons, which can hardly contribute to the free charge carriers. The mobility of organic semiconductors (including crystalline organic semiconductors) is usually much lower than inorganics, because of their localized charges. Moreover, there are few intrinsic charge carriers in organic semiconductors, which makes them poorly conductive without doping. Despite many drawbacks in electronic qualities and semiconducting properties, organic semiconductor have many distinguished advantages, especially 
the good tunability (for example, absorption and emission spectra). Together with their properties of easy process, flexibility and light weight, organic semiconductors are great candidates for special optoelectronic applications.

The photovoltaic effect in organics was observed for the first time by H. Kallmann and M. Pope in 1959[18]. It was not until the 1980s that there was a big breakthrough in organic solar cells, when C.W. Tang reported the first donor-acceptor organic solar cell with a bilayer structure. At that time the power conversion efficiency (PCE) was about $1 \%$. Now, the best organic solar cell has achieved more than $18 \%$ PCE $[19,20]$, which is approaching silicon or perovskite solar cells. Encouraging initial lifetime $(>8,000 \mathrm{~h}$ unencapsulated) has been achieved in commercialized products [21]. However, silicon solar cells have achieved more than $26 \%$ PCE [22, 23], and the performance of commercial modules can keep 95\% after 20-years outdoor usage [24]. Considering the efficiency, cost and stability issues, the status of silicon in the power grid is unshakable. However, just like the application of particular inorganic semiconductor in special fields as mentioned above, organic photovoltaics may also occupy a place in the subdivision of photovoltaics, for example, semi-transparent PV for greenhouse, PV for indoor applications, and PV for mobile devices.

Currently, the commercialization of organic solar cells is already underway. However, there are still many scientific issues that plague academia. For example, controversial studies and debates on the mechanism of charge separation are going on. Moreover, new problems continue to arise with the emergence of new materials, while old problems may fade with the retirement of old materials. For example, the development of non-fullerene acceptors has brought opportunities to reduce the voltage loss and additional requirements for understanding charge separation and recombination, meanwhile, researchers may care less about the tunability of fullerene.

\subsection{Aims and structure of this thesis}

The aim of this thesis is to understand the loss mechanisms in organic solar cells, especially those based on non-fullerene acceptors. The mind map to achieve this goal is exhibited in (Figure 1.3). The thesis focuses on two main fundamental questions on the topic "loss mechanisms of organic solar cells": what is affecting the open-circuit voltage and what is affecting the fill factor.

To answer these questions, we have to be equipped with basic knowledge of semiconductor physics and donor-acceptor organic solar cells (chapter 2), including the origin and the characteristics of the photovoltaic effect, and the special features of organic photovoltaics. After the basic concepts and theory, the loss analysis can be overall analysis of a system based on thermal dynamics, or individual analysis of electronic states. On the one hand, recombination at the open-circuit condition dominates the open-circuit voltage (chapter 3). On the other hand, the charge separation and recombination process as a function of bias influences the current generation and the maximum power point, which is the physical origin of FF (chapter 4). To study those processes in organic solar cells, stead-state and transient techniques are required (chapter 6). The last part of the thesis (chapter 7) summarizes the results of the appended papers. 
Ch. 1 | Introduction

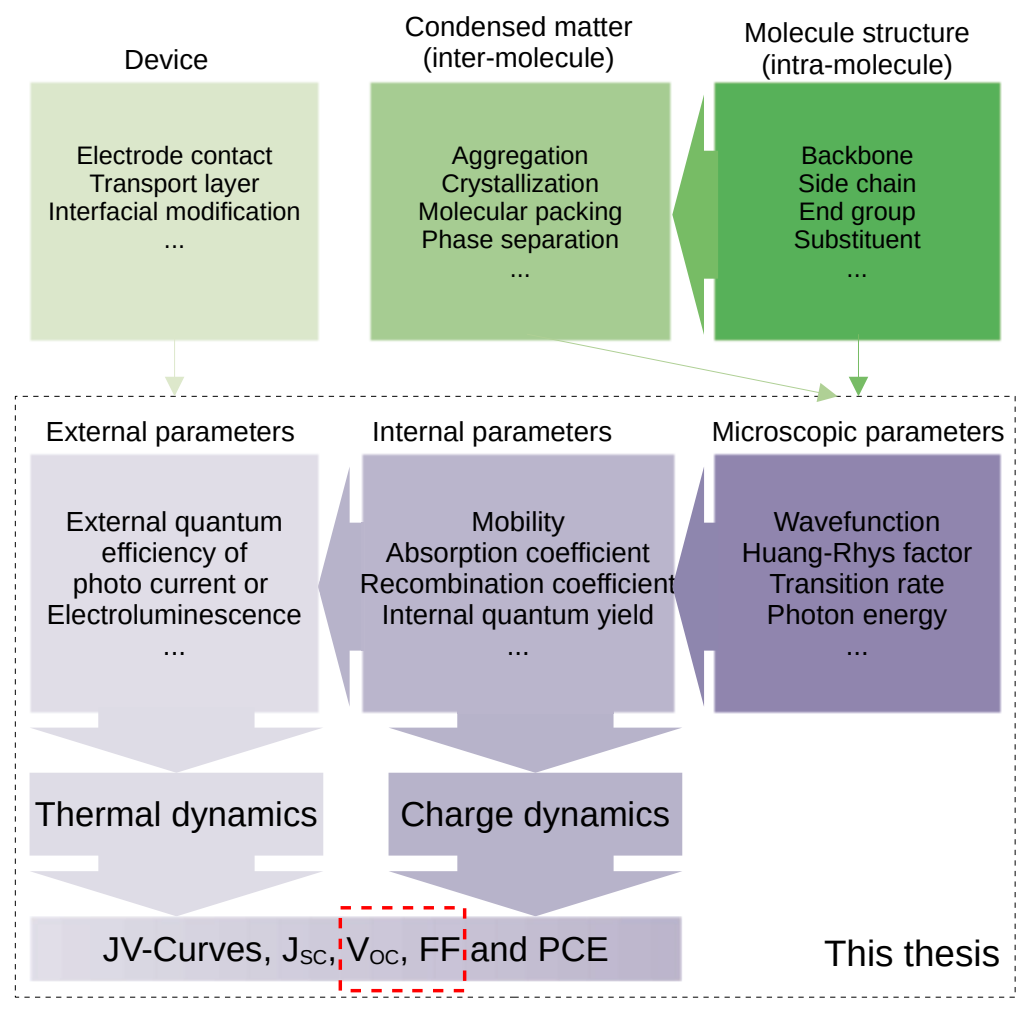

Figure 1.3 | Factors determining the performance of a typical organic solar cell. The terms in green squares are phenomenon or matters, which are mainly dealt with in material synthesis, film formation and device fabrication. The terms in purple squares are physical parameters, which rely on the matters as indicated by the slim green arrows. $\mathrm{FF}$ and $\mathrm{V}_{\mathrm{OC}}$ in the dashed red frame are main investigation objects of this thesis. (This mind map is inspired by [25]) 


\section{CHAPTER 2}

Basic concepts

In this chapter, some basic concepts and theories of semiconductors physics and organic semiconductors for photovoltaics are introduced.

\subsection{The photovoltaic effect}

The word "photovoltaic" consists of "photo" and "voltaic", indicating the straightforward working principle, photons in and voltage out. The realistic working process in a solar cell is more than pure voltage output. From the perspective of energy, it is the conversion of photo energy into electric potential. Here comes the question, what drives the conversion?

\subsubsection{From photonic energy to chemical potential}

The absorption of photons occurs through the excitation of electrons into states of higher energy. As with other optical and electrical properties, the absorption of metals, semiconductors, and insulators can be completely different. The fundamental reason is their difference in electronic structures. With calculation of the wave function in a semiconductor, it is found that the allowed electron energy is not continuous. The ranges of energy levels that electrons may have are called bands and the ranges that they may not have are called band gaps or forbidden bands [26]. In equilibrium conditions, Fermi-Dirac statistics can describe the distribution of electrons over energy states,

$$
f(E)=\frac{1}{\exp \left[\left(E-E_{\mathrm{F}}\right) / k_{\mathrm{B}} T\right]+1}
$$

where $f(E)$ is the occupation possibility of a state with energy $E, k_{\mathrm{B}}$ is Boltzmann constant, $T$ is the temperature, and $E_{\mathrm{F}}$ is the Fermi level which is also the chemical 
potential of electrons.

a

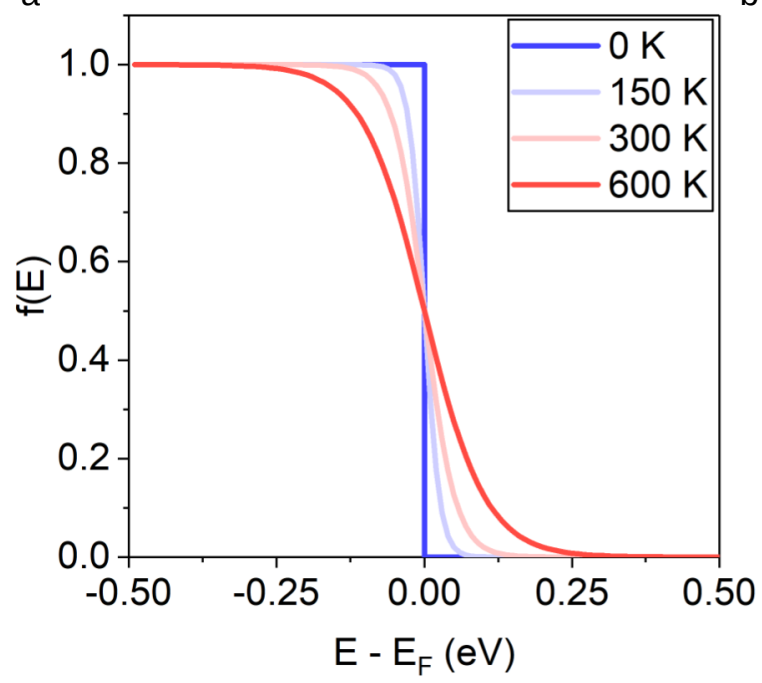

b

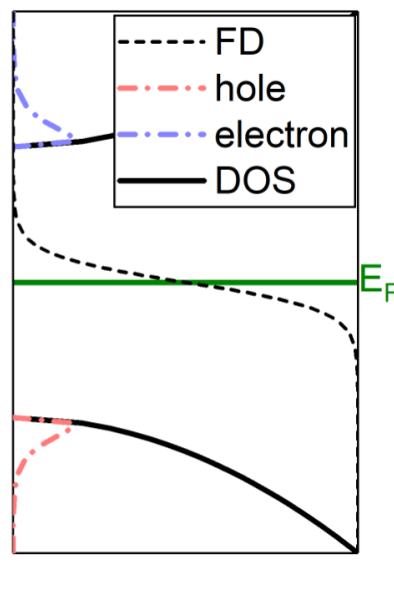

Figure 2.1 | Fermi-Dirac distribution and density of states. a, Fermi-Dirac (FD) statistics at $0,150,300$ and $600 \mathrm{~K}$. The abscissa is energy, and the ordinate is occupation probability. $\mathbf{b}$, Demonstration of electron and hole densities under the FD distribution at $300 \mathrm{~K}$, in conduction and valence bands with the parabolic density of states (DOS). (Credit: Wolfgang Tress.)

As for a semiconductor, the Fermi level lies within the band gap, and the gap is small enough for electrons to be "thermally populated". The bands closest to the Fermi level are called the conduction band and the valence band, both of which have decisive impacts on the conductivity of the semiconductor. At absolute zero, the conduction band is located at the lowest range of empty electronic states, while the valence band is the highest range of occupied electronic states. A solid conducts only if the valence band is not fully occupied or the conduction band is not entirely empty, because the total momentum of electrons may be changed in an electric field, and there would be a net current. The occupation of the density of states can be described in Figure 2.1. The empty states can be treated as quasi-particles, which are called holes. The Fermi level and electron occupation can be changed by doping electron donors or acceptors, which changes the conductivity and chemical potential in equilibrium conditions. Any deviation from the thermal equilibrium changes the level of occupation of the electronic states, hence altering the concentrations of electrons and holes. Light-induced excitation, electrical injection or a sudden increase in temperature can produce fresh charge carriers, creating a non-equilibrium condition.

When semiconductors are illuminated by photons with energy higher than the gap, electrons at the valence band can be excited by absorbing photons. The photo-excited electrons initially reside at an energy higher than the conduction band edge. Since there are many density of states at a lower energy, the hot carriers may thermalize 
within femtoseconds to the band edge by absorbing and releasing phonons. Before they recombine and eventually reach thermal equilibrium, the charge carriers exist in their bands for their "lifetime", which is much longer than their thermalization time [27].

Under continuous illumination, the generation and recombination reach thermal and chemical equilibrium, and a certain amount of charges always remain in the bands. The distribution can still be described by Fermi-Dirac distribution because the thermalization process is short, which originates from the collision between electrons and phonons, while the recombination process is much longer, which originates from the collision between electrons and holes. To describe the occupation of the states in the conduction band and the valence band separately, two quasi-Fermi levels $\left(\mathrm{E}_{\mathrm{f}, \mathrm{n}}, \mathrm{E}_{\mathrm{f}, \mathrm{p}}\right)$ can be used, as shown in Figure 2.2. The splitting of quasi-Fermi levels denotes the increase of chemical potential in the semiconductor.

a

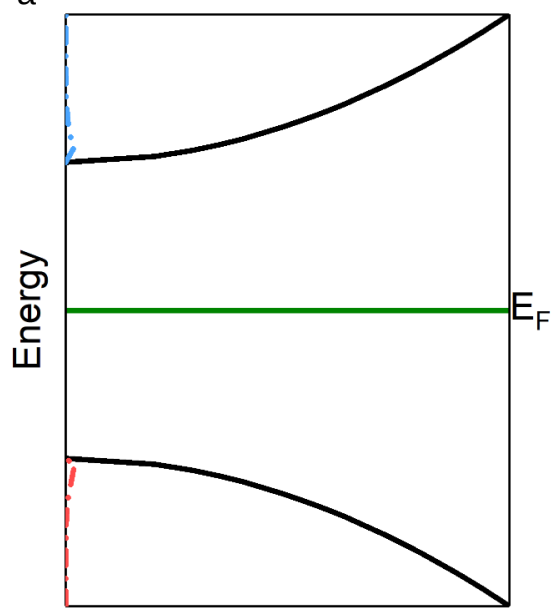

b

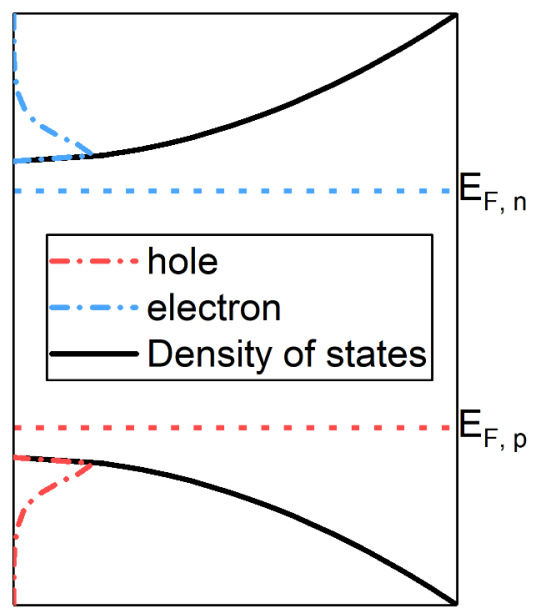

Figure 2.2 | Quasi-Fermi level splitting. a, The distribution of electrons and holes in the DOS in the equilibrium condition. b, Electrons and holes under continuous light illumination, where the density of electrons and holes need to be described by two quasi-Fermi levels, individually.

The equilibrium point may move due to the change of the recombination rate or extraction of the charges. Accordingly, faster recombination or extraction will lead to a lower chemical potential. The detailed process will be discussed in chapter 3 .

\subsubsection{From chemical potential to electrical energy}

In the previous subsection, we have introduced the photo-induced chemical potential change in semiconductors. However, a homogeneous semiconductor cannot produce a voltage output, because voltage requires spatial variations in electric poten- 
tial, for example, a spatial gradient of charges or electrochemical potentials. To create voltage via semiconductors, one of the simplest ways is to make electrical junctions [28].

An electrical junction is a point or area where multiple conductors or semiconductors make physical contact. For example, the homojunction, the heterojunction, and the metal-semiconductor junction. A spatial difference in Fermi level indicates the difference in charge carrier density, which leads to the diffusion of charge carriers from high intensity to low intensity. The diffusion breaks electrical neutrality, creating the space charge region and the electric field. The electric field drives charge carriers to drift back. Only when the electric field is large enough, and the drift current cancels out the diffusion current, the system can reach an equilibrium. The most commonly known solar cell is configured as a p-n junction (for example, $p$-type silicon and n-type silicon). Another commonly used configuration for solution-processed solar cells is $\mathrm{p}-\mathrm{i}-\mathrm{n}$ (or $\mathrm{n}-\mathrm{i}-\mathrm{p}$ ) structure, which consists of $\mathrm{p}$-type hole transport layers, n-type electron transport layers, and an intrinsic semiconductor layer as the light absorber.

To describe the diffusion and drift process in a semiconductor juntion, one can use the following equations,

$$
\begin{array}{r}
\boldsymbol{J}_{\text {diffusion }}=e D_{n} \nabla n-e D_{p} \nabla p \\
\boldsymbol{J}_{\text {driftf }}=e n \mu_{n} \boldsymbol{F}+e p \mu_{p} \boldsymbol{F}
\end{array}
$$

where $\boldsymbol{J}_{\text {diffusion }}$ is the diffusion current, $e$ is the elementary charge, $D_{n}$ or $D_{p}$ is the diffusion constant for electrons or holes, $\nabla$ is the vector differential operator, $n$ or $p$ is the density of electrons or holes, $\boldsymbol{J}_{\text {driff }}$ is the drift current, $\mu_{n}$ or $\mu_{p}$ is the electron or hole mobility, and $\boldsymbol{F}$ is the internal electric field.

Using Einstein relation, the diffusion constant can be transformed and expressed by the mobility of carriers. Using Boltzmann approximation, carrier densities can be expressed as a function of fermi levels [26]. Hence, the total current can be expressed in the following form,

$$
\boldsymbol{J}=\boldsymbol{J}_{n}+\boldsymbol{J}_{p}=n \mu_{n} \boldsymbol{\nabla} E_{F, n}+p \mu_{p} \boldsymbol{\nabla} E_{F, p}
$$

where $\boldsymbol{J}$ is the total current, $\boldsymbol{J}_{n}$ is the electron current, and $\boldsymbol{J}_{p}$ is the hole current. There would be a net electron (or hole) current if there is a quasi-Fermi-level gradient of electrons (or holes). As shown in a simulation result via SCAPS (Figure 2.3 a and d), there is no Fermi-level splitting for a zero-bias p-n junction in the dark. The conduction band and valence band are bent near the boundary between the $\mathrm{p}$ type semiconductor and the n-type semiconductor because of the built-in field and the vacuum level shift. Here, the contacts at both ends are preset to have the same Fermi level (or work function) as that of the semiconductor, which is for flat bands at both ends. When there is continuous light illumination from the left side, quasiFermi levels split and reach quasi-equilibrium. In Figure 2.3 b, where the p-n junction is at the open-circuit condition, there is a significant difference between $E_{F, p}$ at the left end and $E_{F, n}$ at the right end. The quasi-Fermi levels have slight bending, but the electron and hole currents cancel each other out. The difference between them equals $e V_{O C}$. In Figure 2.3 e, the overall charge density is increased but the change 
in minority carriers is more pronounced (note that the asymmetry is caused by the one-side illumination and hence the generation difference between two sides). In Figure $2.3 \mathrm{c}$, where the $\mathrm{p}-\mathrm{n}$ junction is at the short-circuit condition, the conduction and valence band bending is similar to that at the dark condition, while the quasiFermi level splitting is significant and the bending around space charge region (the junction) is notable.
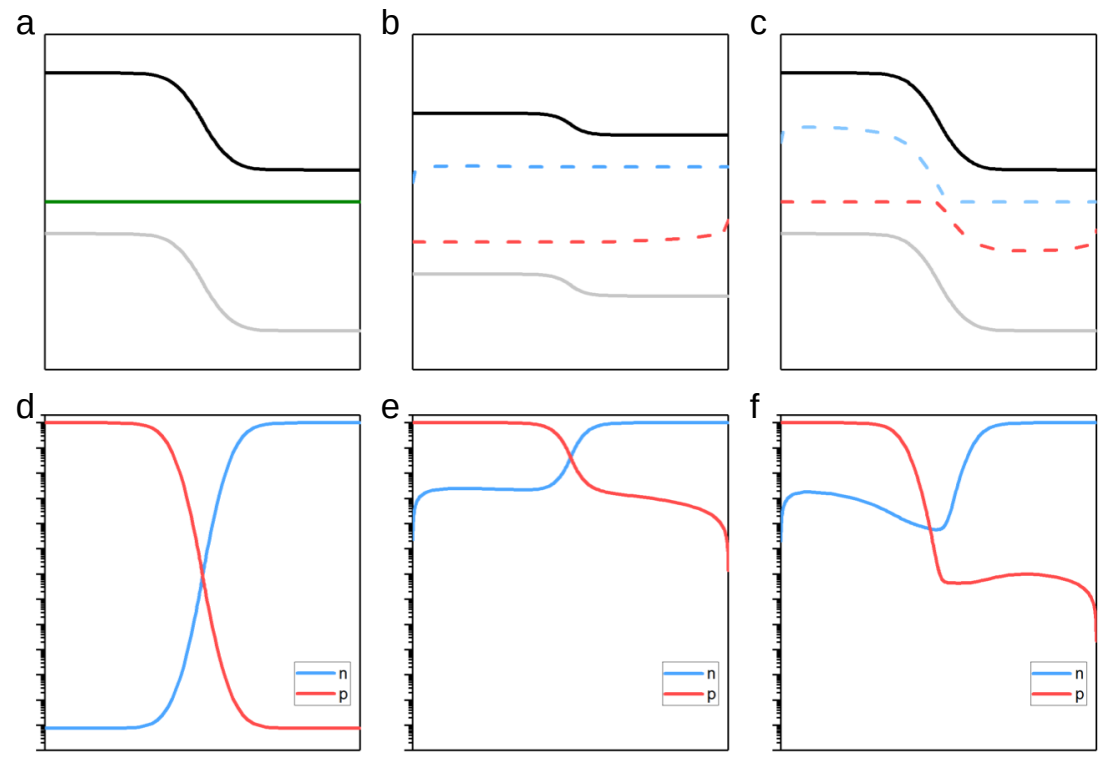

Figure 2.3 | Energy levels and charge densities in a p-n junction. a-c, Energy levels at dark and zero bias condition, light and open-circuit condition, and light and short-circuit condition respectively d-f, Electons and holes density at dark and zero bias condition, light and open-circuit condition, and light and short-circuit condition respectively. The One-dimensional results are simulated through SCAPS, with contacting, with or without illumination from the left side.

A balance of diffusion and drift current is achieved within the junction in the dark state, and the built-in field cancels out the chemical potential difference between the two sides of the junction. Upon light shining, the drift current is enhanced and the equilibrium starts to move. In this case, either the drift current is extracted out of the junction or the charge is accumulated to form a voltage until a new equilibrium. To summarize, the force converting the chemical potential within a semiconductor into electrical potential is the built-in electric field, and the built-in electric field comes from the joint of two sites with different fermi levels, which is a semiconductor junction. No matter it is a p-n junction or a p-i-n junction, the internal field, charge diffusion/drift ability, charge density, and recombination processes have significant impacts on the performance of a solar cell. 


\subsubsection{Characteristics of solar cells}

The performance of a solar cell is commonly characterized by scanning the voltage and measuring the current under illumination. Although solar cells in real-world applications may work in an ever-changing natural environment, academia and industry need a standard to measure their performance. The typical standard test conditions for power output are radiation of Air Mass 1.5 Global (AM1.5G) and a cell temperature of $25^{\circ} \mathrm{C}$. "Air Mass 1.5" means 1.5 atmosphere thickness, corresponding to a solar zenith angle of $48.2^{\circ}$ [29]. "Global" implies the inclusion of both direct and diffuse radiation. The AM1.5G spectrum standard can be found in ASTM G-173, and the spectrum has an integrated power of $1000 \mathrm{~W} / \mathrm{m} 2(100 \mathrm{~mW} / \mathrm{cm} 2)$ [30]. During indoor laboratory testing, solar simulators are usually used to provide controllable illumination approximating standard AM1.5G. Several types of lamps have been used as the light source within solar simulators, including Xenon arc lamps, light-emitting diodes (LED), and metal halide arc lamps. As shown in Figure $2.4 \mathbf{a}, \mathbf{b}$, the spectra of Xenon lamp-based and LED-based simulators are compared with AM1.5G. Here, both simulators meet the class A specification in the spectral match, despite obvious spectral differences between the simulators and AM1.5G [31].
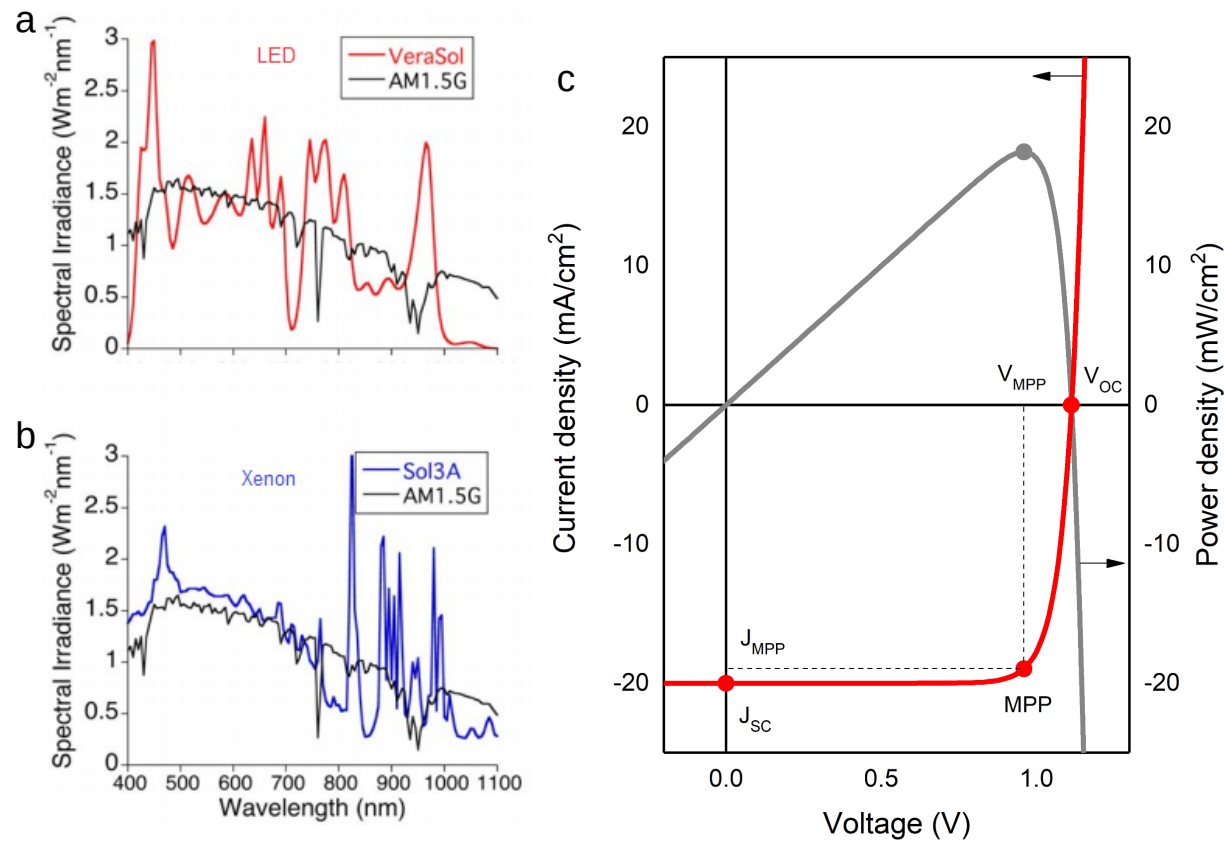

Figure 2.4 | Basic characteristics of a solar cell. Spectral match of (a) the LED (VeraSol) and (b) Xenon (Sol3A) solar simulator to the AM1.5G spectrum. (Reproduced with permission.[31] Copyright 2016, IEEE.) c, The typical current density versus voltage characteristic curve of a solar cell under a solar simulator

Using a sourcemeter (for example, Keithley 2400) to sweep applied voltage and to 
measure current, one can get a current density versus voltage (JV) characteristic curve of a solar cell (Figure 2.4 c). In a short-circuit condition, where the output voltage bias is zero, the external load has zero or negligible resistance. The current density recorded at the short-circuit condition is called short-circuit current density $\left(J_{S C}\right)$. In an open-circuit condition, where no net current flows out of the device, the external load has infinitely or extremely large resistance. The voltage recorded at the opencircuit condition is called open-circuit voltage $\left(V_{O C}\right)$. There is no output power at both short-circuit and open-circuit conditions. The output power reaches a maximum power $\left(P_{\max }\right)$ when the load is optimized, and the maximum power point (MPP) can be extracted from the JV curve when voltage sweep effectively simulates the presence of different loads. The power conversion efficiency (PCE) of a solar cell is defined as the ratio between $P_{\max }$ and the power of the incident light $\left(P_{i n}\right)$,

$$
P C E=\frac{P_{\text {max }}}{P_{i n}}=\frac{J_{M P P} V_{M P P}}{P_{i n}}
$$

where $J_{M P P}$ and $V_{M P P}$ refer to the current density and the voltage at the MPP, respectively.

Another term to describe the overall performance of a solar cell is the fill factor (FF). The fill factor is defined as

$$
F F=\frac{J_{M P P} V_{M P P}}{J_{S C} V_{O C}}
$$

Using FF, PCE is then presented as

$$
P C E=\frac{F F J_{S C} V_{O C}}{P_{i n}}
$$

The benefit of using this expression is that FF, $J_{S C}$ and $V_{O C}$ can be related to the physical properties in a solar cell. For example, $J_{S C}$ depends on how many photo-generated charges can be extracted from the device, and it can be related to the external quantum efficiency of the solar cell. $V_{O C}$ depends on the fermi level splitting and the charge amount staying in the device. FF depends on the competition between charge recombination and extraction. The details regarding $V_{O C}$ and FF is discussed in chapter 3 and chapter 4.

Besides PCE which is a measure of the overall conversion efficiency, quantum efficiency is a spectrally resolved measure of a solar cell's efficiency. The external quantum efficiency (EQE) is the ratio of the amount of charge carriers yielded by a solar cell to the amount of incident photons,

$$
\operatorname{EQE}(\lambda)=\frac{n_{\text {carrier, out }}(\lambda)}{n_{\text {photon, in }}(\lambda)}
$$

where $\lambda$ is the wavelength, $n_{\text {carrier, out }}$ is the number of collected charge carriers per unit time, and $n_{\text {photon, in }}$ is the number of incident photons during the same period. To be distinguished from the EQE of LEDs, the EQE of photocurrent conversion is expressed as $\mathrm{EQE}_{\mathrm{PV}}$ in the following text.

In addition to the characterization performed under illumination, the JV in the dark condition is also an important part of the characteristics of a solar cell. The barrier 


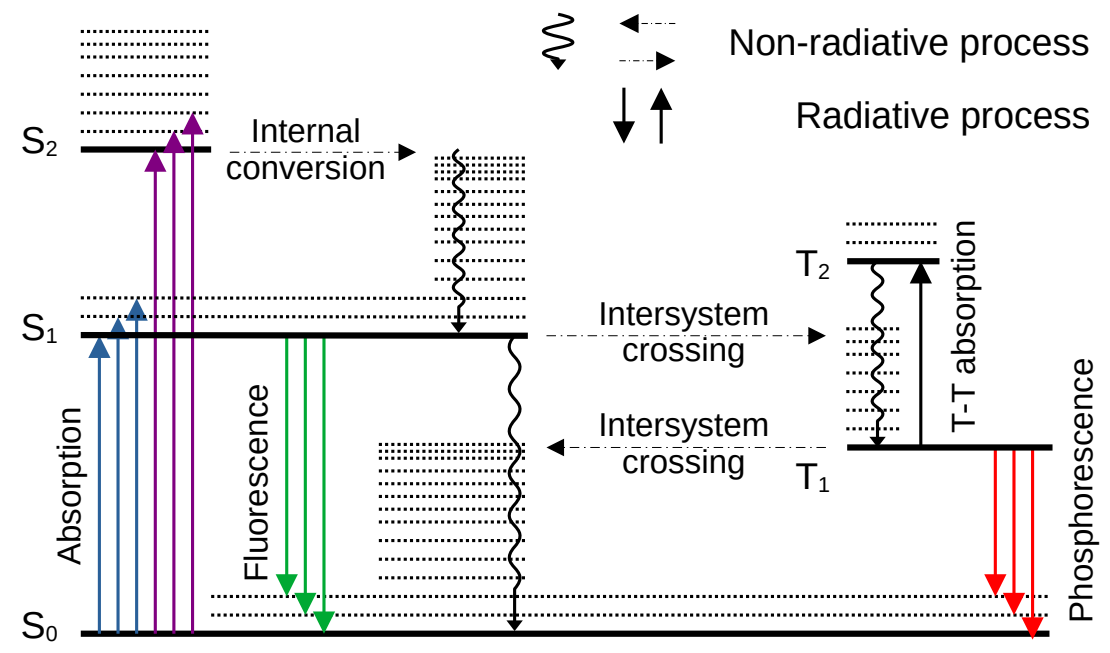

Figure 2.5 | Jablonski scheme with light excitation and decay process. $S_{0}$ is the ground state. $S_{1}$ and $S_{2}$ are the first and the second excited state of singlet. $T_{1}$ and $T_{2}$ are the first and the second excited state of triplet.

and built-in electric field of a junction give a solar cell rectifying characteristics, which means it passes current in one preferred direction. Moreover, the reverse current contains the information about the parallel resistance, the forward current contains the information about diode features and the series resistance.

\subsection{Donor-acceptor organic solar cells}

As mentioned in section 1.2, organic semiconductors are very different from inorganic semiconductors. In organic solar cells, the most interesting phenomenon is that tightly bound charges in single components are hard to be extracted, while specific organic blends generate long-lived free charge carriers which can be collected by electrodes easily. The former occurs due to the energetic features of organic semiconductors, and the latter involves a kind of interfacial state. The details are introduced in the following subsections.

\subsubsection{Energetics of organic semiconductors}

From atoms to molecules, orbitals of atoms hybrid and form new orbitals. This is the same for both organic and inorganic semiconductors. In conventional inorganic 
semiconductors, covalent or ionic bonds are everywhere within a crystal and the electron cloud can extend three-dimensionally. In organics, however, the same interaction may end within a much shorter length than inorganics, and the overlap of orbitals may be limited in certain dimensions. Essentially, without the widespread intermolecular interactions, organics may lose those soft and lightweight properties as well.

Typical organic semiconductors are conjugated molecules. Due to the discontinuous energy and localized wave function, the terms "conduction band" and "valence band" are not suitable for describing energy levels of organic semiconductors[15, 32]. Instead, the highest occupied molecular orbital (HOMO) and the lowest unoccupied molecular orbital (LUMO) are used to analyze and interpret the optical and electrical properties of organic semiconductors. By changing molecular structure, for example, the length of conjugation, the electron withdrawing or donating ability of functional groups, the HOMO and the LUMO can be easily tuned. From benzene (1 ring) to pentacene ( 5 rings), the gap between HOMO and LUMO is decreased by about 3.8 $\mathrm{eV}$, which corresponds to their absorption change [33]. HOMO and LUMO are very important. However, they are not sufficient to describe the entire optical transitions in organics.

To illustrate the electronic states of a molecule and the transition between them, a Jablonski diagram can be used. As shown in Figure 2.5, the states represented by horizontal lines are arranged vertically by energy and grouped horizontally by spin multiplicity [15]. A radiative transition from an energy level to a higher level is mediated by the absorption of a photon, while a transition to a lower level involves the emission of a photon. Non-radiative transitions can occur through vibrational relaxation of the excited state to its lowest vibrational state. This transition process cannot occur for isolated molecules because it involves the dissipation of energy from the molecule to its surroundings. Another kind of radiationless transition is internal conversion (IC), which occurs through the coupling between vibrational states of two electronic states. A third kind of non-radiative transition is intersystem crossing (ISC), which involves two electronic states with different state spin multiplicity and is highly related to the spin-orbit coupling of the molecule. ISC can be followed by phosphorescence.

Molecular bonds are always vibrating and the motion of nuclei causes vibrational energy levels. Vibronic transitions are the simultaneous changes in electronic and vibrational energy levels of a molecule due to absorption or emission of a photon [36]. To explain the intensity of vibronic transition within molecules, one can refer to the Franck-Condon principle. This principle states that an optical transition is most probable for a vertical transition when the nuclear coordinates keep the same position, while two vibrationally relaxed states have a small transition probability due to their different nuclei positions. This is the origin of Stokes shift. From the perspective of quantum mechanics, the probability of radiative transition depends on the wavefunction overlap. Figure 2.6 b is the Schematic diagram of the absorption and emission spectra corresponding to the energy diagram in Figure 2.6 a. An identical shape of the ground and excited-state potential wells lead to the symmetry of absorption and emission spectra.

Organic semiconductors naturally tend to be disordered. Organic polymers have in- 


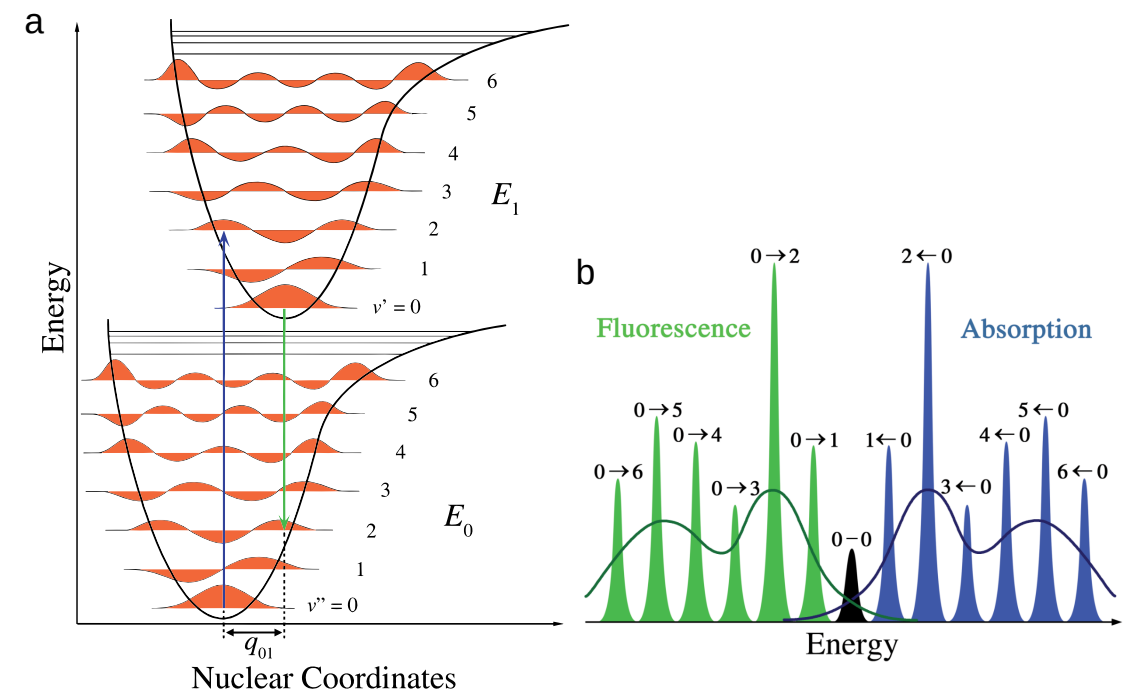

Figure 2.6 | Franck-Condon principle energy diagram. a, The overlap of vibrational wave functions determines the transition propability. Here, the most favored transitions are between $v^{\prime \prime}=0$ and $v^{\prime}=2$ for the absorption (blue line), and between $v^{\prime}=$ 0 and $\mathrm{v} "=2$ for the emission (green line). b, The absorption and fluorescence spectra corresponds to the energy diagram. Isolated narrow peaks usually only appear for dispersed gas molecules, while liquids and solids show inhomogeneous broadening of the same transitions as indicated by the black curves. Transitions between the lowest vibrational levels of the electronic states (the zeroth to zeroth transition) have the same energy in both absorption and emission. Figures by Mark Somoza under CC-BY-SA 4.0 and GFDL license.[34, 35]

homogeneous chain lengths and conformational defects (twists and kinks), resulting in diverses site energies (disorder) [37]. Further disorder can be introduced by the morphology of the whole condensed phase. Crystallinity, aggregation, molecular orientation, and any charged species may affect the energetic landscape of a material. The energetic disorder is thus a collective property of both morphology and chemical structures. A widely assumed distribution of the density of states (DOS) in organic semiconductors is a Gaussian distribution,

$$
g(E)=\frac{N_{0}}{\sqrt{2 \pi \sigma^{2}}} \exp \left(-\frac{\left(E-E_{0}\right)^{2}}{2 \sigma^{2}}\right)
$$

where $N_{0}$ is the concentration of sites, $\sigma$ is the standard deviation of the distribution, $E$ is the energy of sites, and $E_{0}$ is the average energy. A schematic of energy sites along with the space is shown in Figure 2.7, which is randomly generated based on Gaussian distribution. Here, the $\sigma$ is the commonly referred energetic disorder in charge transport simulations. The charge transport between energetic sites will be discussed in chapter 4 . 

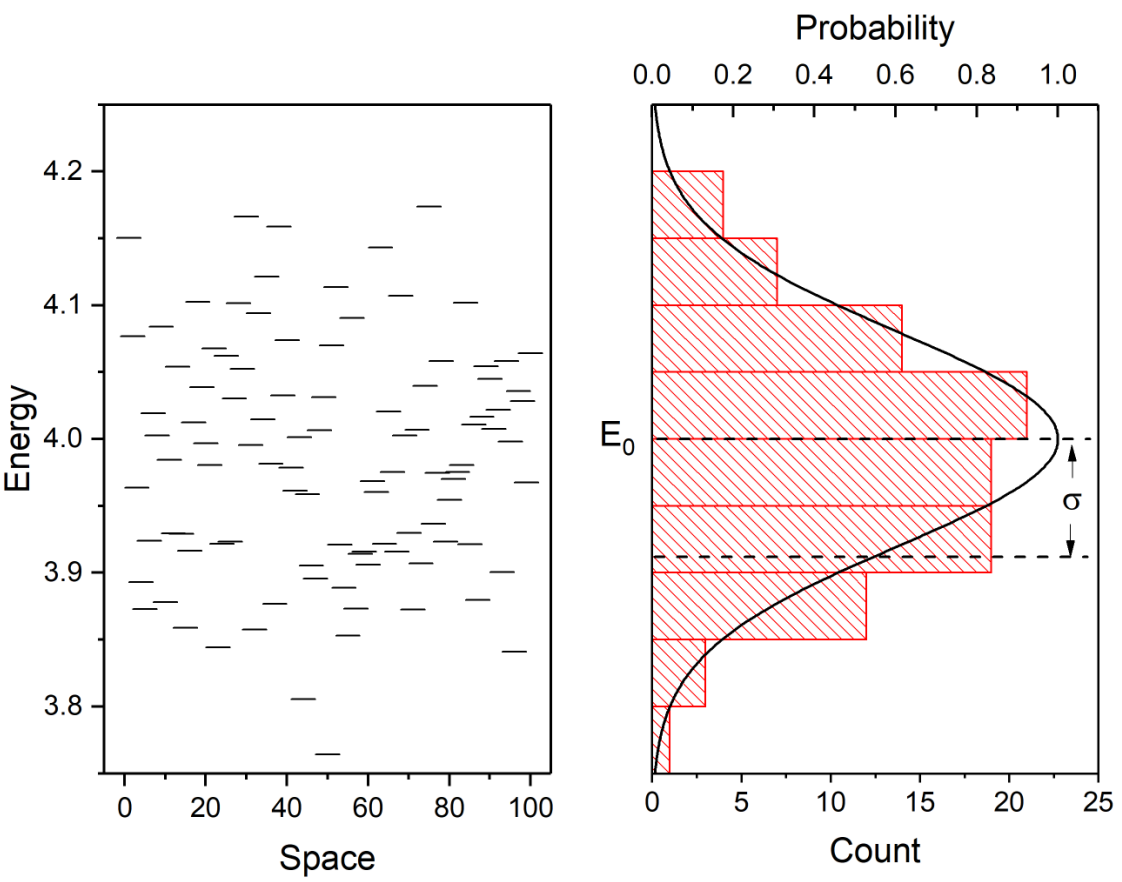

Figure 2.7 | Schematic illustration of energy sites with a Gaussian distribution. The energy of 100 sites along the space are generated randomly following a Gaussian distribution with mean energy $E_{0}$ and standard distribution $\sigma$.

\subsubsection{Interfacial charge-transfer states}

Generally, charge transfer (CT) can happen from electron-donating sites (donor, D) to electron-accepting sites (acceptor, A), within a molecule or between molecules [38, 39], in the ground state, or in the excited state [40-43]. This subsection focuses, however, on the CT in organic solar cells happening in the excited state at D-A interfaces. A state related to the ground state by a CT transition is called the CT state [44]. Such CT states are intermediates in essential electrical processes, for example, charge separation and recombination $[45,46]$.

Due to the weak oscillator strength between the CT state and the ground state, the absorption of the CT state is usually very weak. A CT absorption, 100-1000 times weaker than the pristine material, was detected by the high-sensitive photothermal deflection spectroscopy [47]. The existence of CT was also proved in the spectral-resolvedphotocurrent spectroscopy, by observing additional free-charge-carrier generation at long wavelengths, which is attributed to direct excitation of CT states [47, 48]. Typically, a clear difference between the blend and the pristine material appears at the small energy part of the log-scale sensitive absorption spectra [49]. In addition, radiative transitions from CT states to the ground state have been observed in blends at energies below both the donor and the acceptor emission in photoluminescence 
(PL) [50] and electroluminescence (EL) [51] spectra.

As shown in Figure 2.8, when the donor is excited by photons, the electron can transfer from the LUMO of the donor molecule to the low-lying LUMO of the adjacent acceptor molecule. The remaining hole at the HOMO of the donor and the transferred electron at the acceptor form a CT exciton due to the Coulomb interaction. A similar hole transfer process can happen when the acceptor is excited. Several studies reported that the electron transfer process from donor polymers to acceptor molecules occurs on a timescale of 10 to $100 \mathrm{fs}$, using ultrafast pump-probe spectroscopy [5254]. Moreover, the charge transfer rate was found to be correlated to the frontier energy level difference [40, 54-57].

The energy of the CT state $\left(E_{C T}\right)$ is determined by the frontier orbital energy levels of D and $\mathrm{A}$, and some other material parameters. Despite a formula expression for $E_{C T}$ has been derived for complexes of $\mathrm{D}$-A molecules in solutions using a solvent-continuum model [58], an quantitative description of $E_{C T}$ in a solid state is very hard. Multiple factors, including Coulomb binding energy, polarization, and aggregation-caused delocalization, are expected to influence $E_{C T}$. The energy difference between the lowest-energy excited singlet state, or called local excited (LE) state, and $E_{C T}$ is usually considered as the driving force of the charge transfer. Empirically, a lower limit, around $0.3 \mathrm{eV}$, of energy difference between the LE state and the CT state $\left(\Delta E_{L E-C T}\right)$ is required for the efficient CT in typical polymer:fullerene blends [40]. However, there have been arguments whether a similar driving force is needed in some non-fullerene blends, to accomplish efficient CT and charge dissociation [49, 59-62].

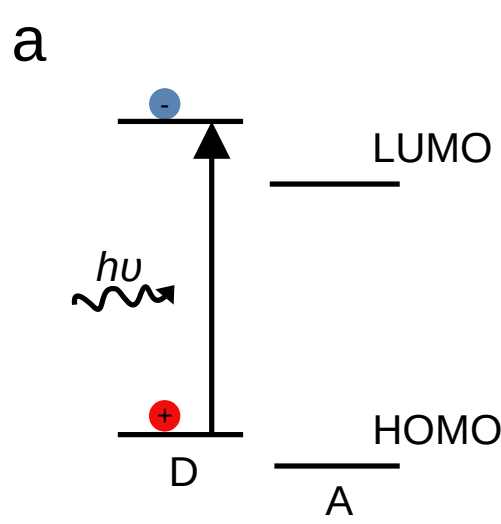

b

Figure 2.8 | Charge transfer. Schematic illustration of an electron transfer process from the LUMO of the donor (D) to the LUMO of the acceptor (A). 


\section{CHAPTER 3}

\section{Thermal dynamics and the voltage loss}

From the perspective of energy conversion, as shown in chapter 2, the operation of a solar cell is the process of converting photonic energy into chemical energy and subsequently into electrical energy. During the processes, the solar cell and its surrounding environment obey the conversion of energy and thermal dynamic principles. This allows us to theoretically calculate the upper limit, as well as the losses of a solar cell's performance. This chapter introduces the detailed balance in solar cells. Later, the voltage loss in solar cells is discussed. Finally, the latest studies on reducing the voltage loss in organic solar cells are reviewed.

\subsection{Detailed balance}

The operation of a solar cell involves the sun, the cell, the environment and the output load. Energy and matter cannot be created or destroyed. They conserve and form a balance within the system.

\subsubsection{Solar spectrum and the black-body radiation}

Any object above absolute zero emits electromagnetic radiation, which is called thermal radiation, or the black-body radiation for an idealized opaque, non-reflective black body. It has a specific shape of spectrum depending only on the temperature of the object. The spectrum can be described by Planck's law due to the quantized nature of light. Planck's law states that

$$
I(E, T)=\frac{2 E^{3}}{h^{3} c^{2}} \frac{1}{\exp \left(\frac{E}{k_{B} T}\right)-1}
$$




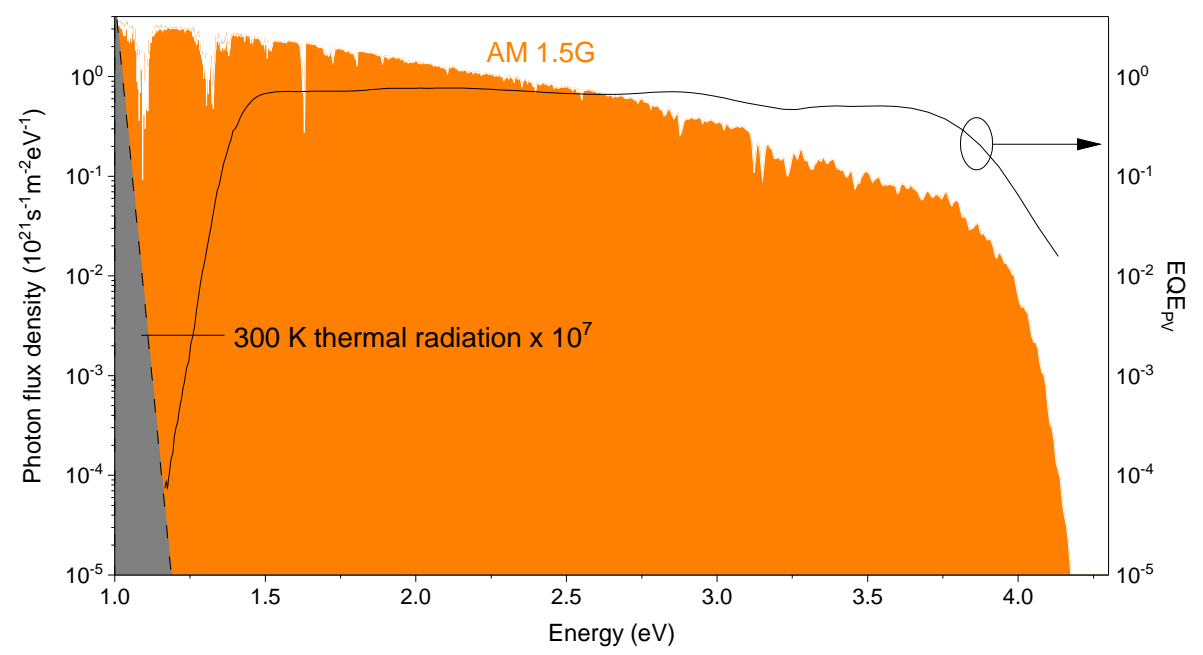

Figure 3.1 | The solar and the blackbody radiation. The standard AM1.5G solar spectrum as well as the $300 \mathrm{~K}$ blackbody radiation expressed in photon flux as a function of energy. The dark blue curve is the EQE of PM6:Y6 with an optical gap around $1.40 \mathrm{eV}$.

where $I(E, T)$ is the equilibrium spectral radiance (the power per unit solid angle and per unit of area normal to the propagation) density of energy $E$ per unit energy at temperature $T, h$ is the Planck constant, $c$ is the speed of light in vacuum, $k_{B}$ is the Boltzmann constant, and $\mathrm{T}$ is the absolute temperature of the body. Our surrounding environment emits thermal radiation due to the background temperature. Matters within the environment emit and absorb electromagnetic radiation, and may eventually reach an equilibrium.

The energy potentially harvested by a solar cell comes from the thermal radiation of the sun, which is to very similar to the radiation of a black body radiator at about $5780 \mathrm{~K}$ [63]. As it passes through the atmosphere, solar radiation is attenuated by scattering and absorption. As mentioned in the previous chapter, the standard solar spectrum for solar cell characterization is AM1.5G. As shown in Figure 3.1, the solar spectrum is expressed by the photon flux as a function of the energy. Many gaps in the spectrum are caused by specific gases, such as water vapor and oxygen. Note that the solar spectrum has a considerable density of photons in the near-infrared (NIR) region, which might be counterintuitive because the solar irradiance as a function of the wavelength has the maximum power density at around $500 \mathrm{~nm}$ (Figure 2.4). This is because, firstly the wavelength interval does not correspond to a constant energy interval, and secondly the energy of photons varies at wavelengths. As a result, the $E Q E_{P V}$ at the infrared region can affect the short-circuit current of a solar cell greatly. 

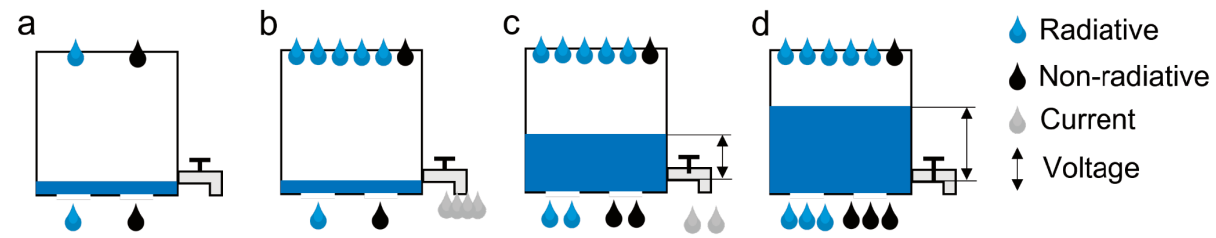

Figure 3.2 | The analogy for charge generation, recombination and output.

a, In the dark condition, radiative recombination equals radiative generation. A similar equilibrium occurs for non-radiative processes. There is no current nor voltage output. b, In the short-circuit condition under illumination, all the photogenerated charges are extracted. c, With light illumination, when a voltage bias is maintained in the working condition, a part of the photogenerated charges are output and the other part becomes additional recombination. d, All the charges recombine in the open-circuit condition.

\subsubsection{Shockley-Queisser limit}

In 1961, Shockley and Queisser initially proposed a theory to derive a thermodynamic limit for solar energy conversion efficiency of photovoltaic cells based on the detailed balance principle. The theory is valid for both inorganic and organic single-junction solar cells. Several fundamental assumptions were proposed for an ideal solar cell: (1) Semiconductor absorbs all photons above the optical gap (Note that it is "band gap" in the original paper. Here, it is adapted to "optical gap" for a unified description of organic and inorganic semiconductors.), and no absorption below the optical gap, which is also called a step function like absorption; (2) Each absorbed photon can only generate one electron-hole pair; (3) Perfect charge transport and collection.

As shown in Figure 3.2, a complete process starts from radiative or non-radiative generation and ends in radiative or non-radiative recombination (note that the output is recombination outside the solar cell). Thus, an equation is built,

$$
F_{S}+R(0)=I / q+F_{C}(V)+R(V)
$$

where $F_{S}$ is the photon flux of solar radiation, $R(V)$ is the non-radiative recombination current of voltage $V, R(0)$ is the non-radiative recombination current at zero voltage, $I$ is the output current, $q$ is the elementary charge, $F_{C}(V)$ is the radiative recombination current of voltage $V$. The left side of the equation is the generation and the right side is the recombination. Here, some transformation has been made. For example, the non-radiative generation has been replaced by $R(0)$ because they cancel out each other at zero bias. For an ideal diode, the equation becomes

$$
I=q\left(F_{S}-F_{C}(0)\right)-q\left(F_{C}(0)+R(0)\right)(1-\exp (q V / k T))
$$

where an ideal factor of 1 has been assumed. This equation describes the output current as a function of the applied voltage bias. Given a specific optical gap and the environment temperature, the only uncertain parameter $\mathrm{R}(0)$ can be deduced from the luminescence efficiency. If the $E Q E_{E L}$ is $100 \%$, there is no non-radiative recombination nor $\mathrm{R}(0)$, enabling a quantitative relation between I and $\mathrm{V}$ for the theoretical 
limit conversion. A series of limits can be obtained when the energy of the optical gap changes. The limits are usually referred to as the Shockley-Queisser (SQ) limit, often expressed in terms of several parameters, including the $\mathrm{V}_{\mathrm{OC}}$, the $J_{S C}$, the $F F$ and the $P C E$, as a function of the energy (Figure 3.3).
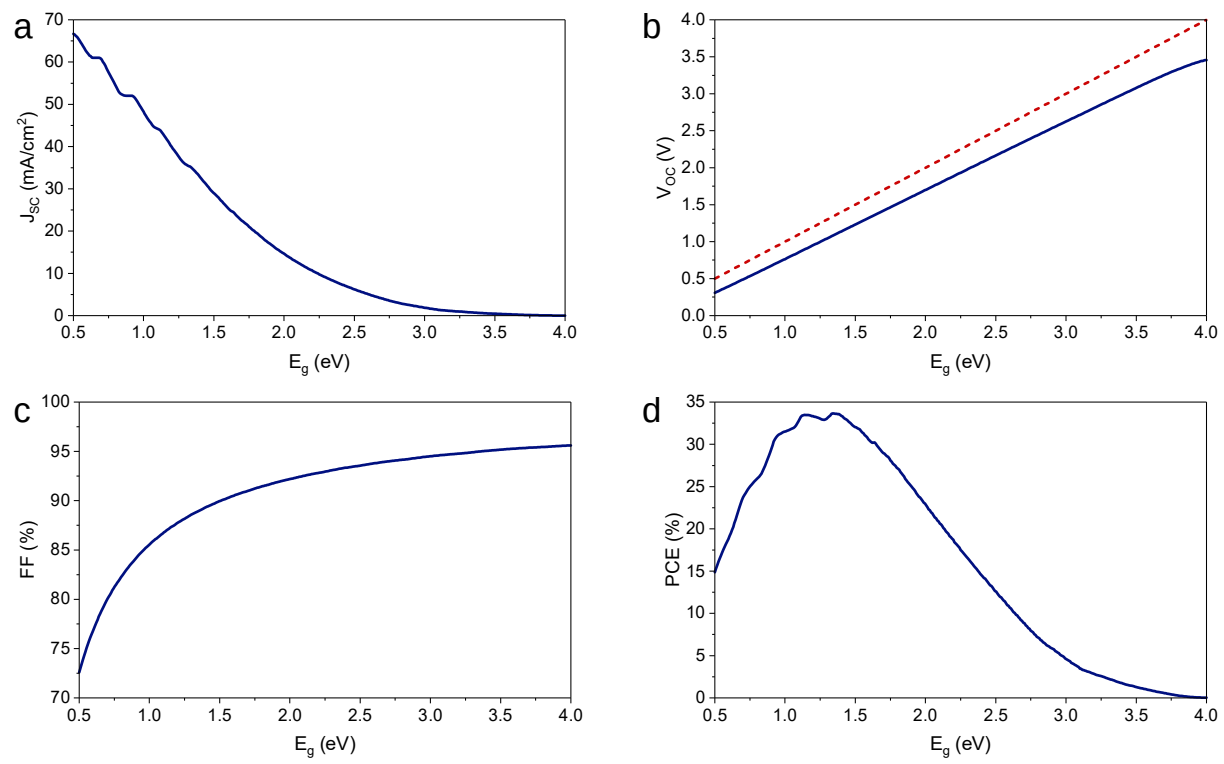

Figure 3.3 | Schematic illustration of the Shockley-Queisser limit. The theoretical limits of any type of single junction solar cells as a function of gap energy are shown in (a) the short-circuit current, (b) the open-circuit voltage, (c) the fill factor, and (d) the power conversion efficiency

\subsubsection{Reciprocity between absorption and emission}

We have shown the equilibrium of charge generation and recombination. Below, we discuss the equilibrium between light absorption and emission.

Kirchhoff's law states that: For a body of any arbitrary material emitting and absorbing thermal electromagnetic radiation at every wavelength in thermodynamic equilibrium, the ratio of its emissive power to its dimensionless coefficient of absorption is equal to a universal function only of radiative wavelength and temperature [64]. In simpler language: For an arbitrary body emitting and absorbing thermal radiation in thermodynamic equilibrium, the emissivity is equal to the absorptivity[64].

A solar cell also absorbs and emits radiation all the time. An analogous reciprocity relation between the photovoltaic and the electroluminescence properties of solar 
cells have been proposed and verified [65-68].

$$
\begin{aligned}
E Q E_{E L}(E) & =\frac{\phi_{\text {emission }}(E, V)}{J(V)} \\
& =\frac{\phi_{\text {emission }}(E, 0)\left(\exp \left(\frac{e V}{k_{B} T}\right)-1\right)}{J_{0}\left(\exp \left(\frac{e V}{k_{B} T}\right)-1\right)} \\
& =\frac{e E Q E_{P V}(E) \phi_{B B}(E)}{J_{0}}
\end{aligned}
$$

where $E Q E_{E L}(E)$ is the electroluminescence quantum efficiency of energy $E$ per unit energy, $\phi_{\text {emission }}(E, V)$ is the solar cell emission photon flux of energy $E$ at bias $V$ per unit energy, $J(V)$ is the current flow at bias $V, J_{0}$ is the total recombination current in the dark equilibrium condition, $e$ is the elementary charge, $k_{B}$ is the Boltzmann constant, $E Q E_{P V}(E)$ photocurrent quantum efficiency of energy $E$, and $\phi_{B B}(E)$ is the thermal radiation of the solar cell of energy $E$.

The significance of this reciprocity includes, that 1) electroluminescence emission and photovoltaic quantum efficiency are quantitatively linked following the detailed balance (Equation 3.4); 2) light-emitting diode quantum efficiency can be used for $\mathrm{V}_{\mathrm{OC}}$ analysis (Equation 3.5); 3) the emission and the absorption spectra can validate the overlapped range and complement the missing parts of each other (Figure 3.4); and 4) it emphasizes that a good solar cell is also a good light-emitting diode.

Equation 3.4 provides a relationship between $J_{0}$ and the quantum efficiency. Thus, the $\mathrm{V}_{\mathrm{OC}}$ can be expressed as

$$
\begin{aligned}
V_{O C} & =\frac{K_{B} T}{e} \ln \left(\frac{J\left(V_{O C}\right)}{J_{0}}+1\right) \\
& =\frac{K_{B} T}{e} \ln \left(\frac{J\left(V_{O C}\right)}{\frac{\int E Q E_{P V}(E) \phi_{B B}(E) d E}{\int E Q E_{E L}(E) d E}}+1\right) \\
& =\frac{K_{B} T}{e} \ln \left(\frac{\int E Q E_{P V}(E) \phi_{s u n}(E) d E}{\frac{1}{E Q E_{E L}} \int E Q E_{P V}(E) \phi_{B B}(E) d E}+1\right) \\
& \approx \frac{K_{B} T}{e} \ln \left(E Q E_{E L} \frac{\int E Q E_{P V}(E) \phi_{s u n}(E) d E}{\int E Q E_{P V}(E) \phi_{B B}(E) d E}\right) \\
& =\frac{K_{B} T}{e} \ln \left(\frac{\int E Q E_{P V}(E) \phi_{s u n}(E) d E}{\int E Q E_{P V}(E) \phi_{B B}(E) d E}\right)+\frac{K_{B} T}{e} \ln \left(E Q E_{E L}\right) \\
& =V_{O C, r a d}+\frac{K_{B} T}{e} \ln \left(E Q E_{E L}\right)
\end{aligned}
$$

where $\phi_{\text {sun }}(E)$ is the solar emission photon flux of energy $E, E Q E_{E L}$ is the overall electroluminescence quantum efficency of all wavelength. $V_{O C}=V_{O C, \text { rad }}$ when there is only radiative recombination and $E Q E_{E L}=1$. This equation helps with the analysis of voltage losses, especially non-radiative loss, which will be discussed in the following section. 


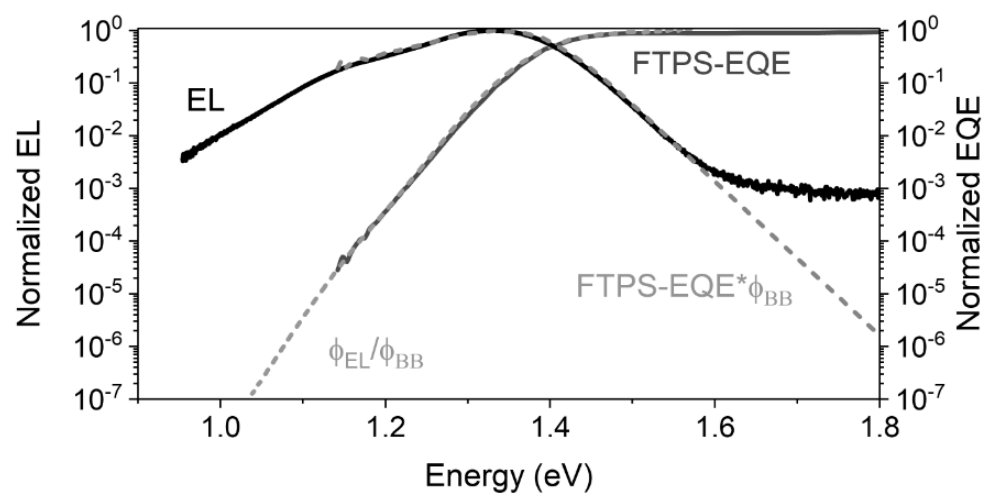

Figure 3.4 | Spectral reciprocity. Normalized EQE ${ }_{P V}$ and EL spectra of the device based on PM6:Y6 are plotted in black. The dashed curves in grey are calculated according to the reciprocity principle, which overlap the measured ones. The ratio of $\phi_{E L}$ and $\phi_{B B}$ was used to calculate the EQE while the product of FTPS-EQE and $\phi_{B B}$ was used to calculate the EL, where $\phi_{E L}$ and $\phi_{B B}$ represent the EL photon flux and the black-body-radiation photon flux at room temperature, respectively.

\subsection{Voltage losses in single-junction solar cells}

Voltage loss is the loss that occurs when a solar cell maintains electrons in high energy states (or charge carrier density) at the open-circuit condition. To better understand this, one can refer to the description in subsection 2.1.2, which indicates the nature of voltage in a solar cell is the difference of the quasi-fermi level at two ends.

In a narrow sense, "voltage loss" refers to the voltage difference between the optical gap over elementary charge $\left(E_{g} / e\right)$ of a solar cell and its open-circuit voltage $\left(\mathrm{V}_{\mathrm{OC}}\right)$. Broadly speaking, it can be the difference of an ideal $\mathrm{V}_{\mathrm{OC}}$ and a more realistic $\mathrm{V}_{\mathrm{OC}}$ (for example, non-radiative voltage loss). The most ideal $\mathrm{V}_{\mathrm{OC}}$ is determined by the intrinsic gap of a material and the unavoidable thermal dynamic processes, which is well described in the previous chapter. Once the optical gap is determined, the upper limit of $\mathrm{V}_{\mathrm{OC}}$ is also determined with idealized assumptions. Moreover, imperfect features of realistic devices cause additional losses and lower $\mathrm{V}_{\mathrm{OC}}$. In general, this section describes the analysis of voltage losses in any type of single-junction solar cells.

\subsubsection{Defining an optical gap}

The optical gap is the threshold for photons to be absorbed. In the SQ limit, the optical gap is described as the turning point where EQE changes from 1 to 0 . However, "turning point" is "turning points" in reality. There are multiple interpretations and analysis methods of this vague "threshold" point. Some may be the point where absorption just begins, while some others may be the point with significant photocurrent remaining, which makes it difficult to compare the voltage losses of solar cells. There- 
fore, to better understand the physical meaning of the optical gap, and to choose an appropriate method for voltage loss analysis, several commonly used methods and their characteristics are discussed below.

One of the most widely used "optical gap" for semiconductors comes from the intersection of the extrapolation of the absorbance edge and the horizontal axis or the extrapolation of the absorbance tail (Figure 3.5 a) [69-71]. This method is easy to use because it only requires an absorbance measurement and the analysis is straightforward. However, a manual extrapolation is arbitrary and may introduce some errors. In addition, the absorbance edge can significantly change once made into a device. On the one hand, conditions (e.g. additional components or annealing) during processing can change the condensed state of the film $[69,72]$, and on the other hand, the structure (e.g. optical cavity) of the device also changes its light absorption [73, 74]. Moreover, determining the optical gap this way lacks physical meaning and overlooks the steepness of the absorption edge when calculating voltage losses, especially not fair for devices that exhibit a sharp edge. Therefore, this method is well suited as a preliminary comparison method, for example, during the synthesis stage of materials, but not appropriate for voltage loss analysis.

An alternative method with more definitive estimation of the optical gap adopts the intersection of the normalized absorption and emission spectra (Figure 3.5 b) [49, 75]. The feasibility of this method is based on that the zero to zero transitions have the same energy in both absorption and fluorescence, as shown in Figure 2.6. However, despite its straightforwardness, there might be a few difficulties. For example, it might be tricky to normalize a spectrum with multiple peaks when the absorption and emission spectra are asymmetrical (In Figure $3.5 \mathrm{~b}$, imagine that the absorption peak at $1.7 \mathrm{eV}$ becomes much higher, almost containing the peak at $1.55 \mathrm{eV}$, and the emission remains the same.). Moreover, if the absorption and emission spectra are not obtained from the device but a film, the same problems mentioned in the previous method occur. Nevertheless, determining the optical gap at the intersection of absorption and emission is physically motivated and usually well reproducible [76]. A similar method is used to define the CT state energy by the absorption and emission spectrum of CT states in organic solar cells.

Recent reports also proposed to determine the optical gap from the EQE spectrum. One method is to use the crossing point of the extrapolated line of the EQE edge and the horizontal tangent of the EQE peak(Figure 3.5 c) $[77,78]$. In sharp contrast to the first, this method takes a point with a significant photocurrent remaining as the optical gap, maximizing the assessment of the steepness of the absorption edge during the voltage loss calculation. The other method treats a solar cell as a superposition of multiple ideal solar cells, by dividing the actual EQE into an infinite number of stepfunction-like EQE of various optical gaps [25, 76, 79]. The gaps form a distribution $P(E)$ along energy which can be obtained by taking the derivative of the EQE with respect to energy (Figure $3.5 \mathrm{~d}$ ). A widely used way to assign an opitcal-gap value is

$$
E_{g}=\frac{\int_{a}^{b} P(E) E d E}{\int_{a}^{b} P(E) d E}
$$

Here, the boundary a and $\mathrm{b}$ are selected where $P(a)=P(b)=0.5 \max [P(E)]$, aiming to exclude the influence of noisy data and negative value of $P(E)$. Overall, determin- 

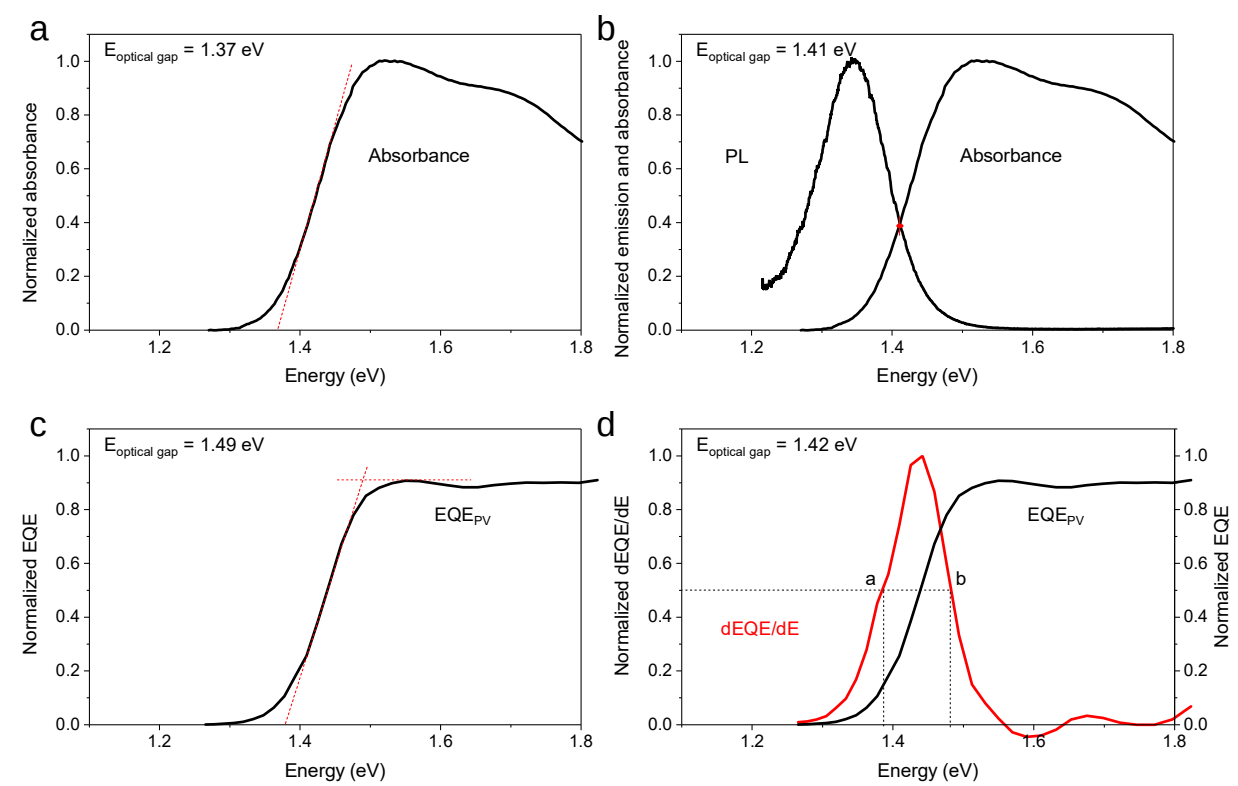

Figure 3.5 | Methods for determing the optical gap. The optical gap can be extracted from (a) the onset of the absorption spectrum, (b) the intersection between the normalized absorption and emission spectra, (c) the intersection of the extrapolated EQE edge and the EQE isoline which passes through the local EQE peak at the edge of the EQE spectrum, and (d) the distribution of optical gaps

ing an optical gap from the EQE spectrum is a quantitative and reproducible method, with physical meaning. It is not only related to the internal characteristics of the absorbing material but also depends on the device structure (the thickness of the active layer, the optical characteristics of the interlayers, and the electrodes). This method requires device fabrication, EQE measurement and calculation, which seems a large workload. However, considering that many reports on organic solar cells have provided the EQE data, this is a very good method for summarization and comparison. Depending on the characteristics of these methods, researchers can choose the one that is suitable for a particular occasion. However, it is essential to use a consistent method to obtain the optical gap values during a comparison. Additional attention to the physical meaning of the optical gap is also necessary when calculating the voltage loss.

\subsubsection{Quantifying voltage losses}

Given the optical gap value $E_{g}$, the total voltage loss can be obtained by subtracting the $\mathrm{V}_{\mathrm{OC}}$ from the $E_{g} / e$. But why do we compare the $\mathrm{V}_{\mathrm{OC}}$ with the gap? That is because optical gaps have fundamental but homogeneous influences on $\mathrm{V}_{\mathrm{OC}}$. After excluding the homogeneous influences, we can analyze the different physical processes remaining and attribute the loss channels to the undesirable properties of materials or 
devices. As shown in Figure 3.6, the total loss can be divided into three parts,

$$
\begin{aligned}
\Delta V_{\text {loss }} & =E_{g} / e-V_{O C} \\
& =\left(E_{g} / e-V_{O C}^{S Q}\right)+\left(V_{O C}^{S Q}-V_{O C}^{\text {rad }}\right)+\left(V_{O C}^{\text {rad }}-V_{O C}\right) \\
& =\left(E_{g} / e-V_{O C}^{S Q}\right)+\Delta V_{O C}^{\text {rad }, \text { below gap }}+\Delta V_{O C}^{\text {non-rad }} \\
& =\Delta V_{1}+\Delta V_{2}+\Delta V_{3}
\end{aligned}
$$

where e is the elementary charge, $\Delta V_{\text {loss }}$ is the total voltage loss, $E_{g}$ is the optical gap, $V_{O C}^{S Q}$ is the maximum voltage according to the Shockley-Queisser limit, $V_{O C}^{r a d}$ is the open-circuit voltage where only radiative recombination occurs, $\Delta V_{O C}^{\text {rad, below gap is }}$ the voltage loss due to the sub-gap absorption induced radiative recombination, and $\Delta V_{O C}^{\text {non-rad }}$ is the voltage loss of the non-radiative recombination.

$\Delta V_{1}\left(E_{g} / e-V_{O C}^{S Q}\right)$ is due to radiative recombination originating from the absorption above the gap, which is unavoidable for any single-junction solar cell. As shown in Figure 3.3, $\Delta V_{1}$ is a function of $E_{g}$. When the $E_{g}$ changes from $1 \mathrm{eV}$ to $2 \mathrm{eV}$, the $\Delta V_{1}$ changes from $0.24 \mathrm{~V}$ to $0.30 \mathrm{~V}$.

$\Delta V_{2}\left(\Delta V_{O C}^{r a d, \text { below gap }}=V_{O C}^{S Q}-V_{O C}^{r a d}\right)$ is due to the additional radiative recombination from the absorption below the optical gap. According to the reciprocity relation, the radiative recombination is directly proportional to the photon absorption of the room-temperature blackbody radiation. As shown in Figure 3.1, the roomtemperature blackbody radiation at the low-energy region is much stronger than that in the high-energy region. The analytical expression for $V_{O C}^{r a d}$ is shown in Equation 3.5. As a result, any sub-gap absorption can cause a large radiative loss even though it may hardly contribute to the photocurrent. For inorganic or perovskite solar cells with steep absorption edges, $\Delta V_{O C}^{\text {rad, below gap }}$ is negligible. Therefore, a steep absorption edge is preferred to avoid this part of loss.

$\Delta V_{3}\left(\Delta V_{O C}^{\text {non-rad }}=-\frac{k T}{e}\left(E Q E_{E L}\right)\right)$ arises from the non-radiative recombination. As also shown in Equation 3.5, $E Q E_{E L}$ is the electroluminescence quantum efficiency of the solar cell when charge carriers are injected into the device in the dark. The enhanced $E Q E_{E L}$ makes it possible to achieve low non-radiative energy losses. In other words, a good solar cell with low non-radiative recombination loss should also be a good light-emitting diode.

\subsection{Reducing voltage losses in organics solar cells}

Despite the increase in PCE, the $\mathrm{V}_{\mathrm{OC}}$ loss, especially the non-radiative loss, is still a substantial challenge for organic solar cells when compared to inorganic or hybrid solar cells. Recently, researchers devoted many efforts to understanding and decreasing the $\mathrm{V}_{\mathrm{OC}}$ loss of organic solar cells, and achieved remarkable results. Some of the understandings are presented in the following subsections. 


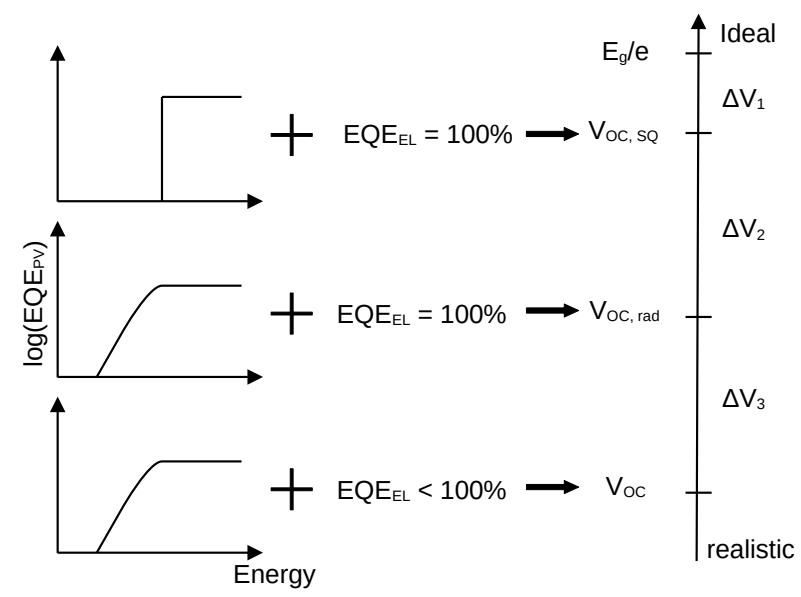

Figure 3.6 | Schematic illustration of voltage losses. An ideal solar cell has stepfunction-like $\mathrm{EQE}_{\mathrm{PV}}$ and $\mathrm{EQE}_{\mathrm{EL}}$ of $100 \%$. A realistic solar cell has $\mathrm{EQE}_{\mathrm{PV}}$ with absorbing features extending to below the gap, which is attributed to the distribution of DOS or specific sub-gap states. These weakly absorbing features lead to additional radiative recombination and voltage losses. A realistic solar cell also has $\mathrm{EQE}_{\mathrm{EL}}$ below $100 \%$, which results in non-radiative voltage losses.

\subsubsection{Energy gap law}

In the condensed phase, polyatomic molecules with narrow gaps tend to exhibit low emission efficiency. This quenching phenomenon and its non-radiative deactivation pathway have been recognized as the 'energy gap law' [80-82]. This law indicates that the relaxation of an excited state to a ground state can be promoted by the wavefunction overlap between the zero vibration level of the excited state and the higher iso-energetic vibration levels of the ground state, subsequently rapid thermalization to the zeroth vibration level of the ground state. This vibrational relaxation accelerates if the emissive states possess low energy and hence large wave-function overlap, drastically reducing the emission intensity, as evidenced by the lack of organic lightemitting diodes in the deep red and NIR region [82, 83].

A recent study shows that the 'energy-gap law' also works for organic solar cells with a trend that the non-radiative voltage loss increased with decreasing CT energy of fullerene-based solar cells (Figure 3.7) [84]. In the study, non-radiative recombination was attributed to intramolecular vibrations of the organic molecules. This model implied that high vibrational carbon-carbon frequency modes have a significant impact on non-radiative recombination. Based on the model, a visible-light-emitting D-A system with simultaneous occurrence of a high photovoltaic and emission quantum yield was reported [85]. The strongly enhanced emission indicates an effective way to reduce $\Delta V_{3}$ by increasing $\mathrm{E}_{\mathrm{CT}}$ and reducing electron-phonon coupling. However, this model cannot explain the performance of some non-fullerene solar cells 

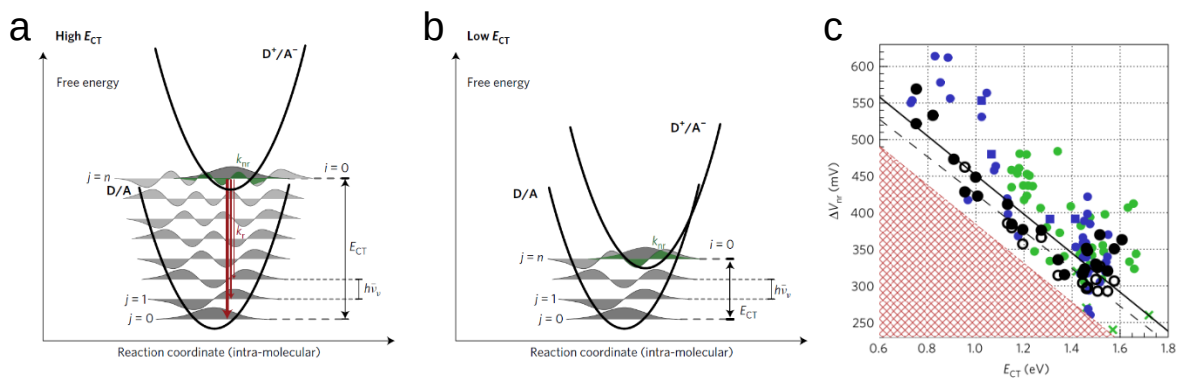

Figure 3.7 | Schematic illustration of the energy gap law. Non-radiative $\left(k_{n r}\right)$ and radiative $\left(k_{r}\right)$ transitions from the CT state to the ground state are demonstrated for (a) a large $\mathrm{E}_{\mathrm{CT}}$ and for (b) a small $\mathrm{E}_{\mathrm{CT}}$. The non-radiative transition is facilitated by the wave-function overlap between the relaxed CT state $(i=0)$ and the vibrationally excited ground state $(j=n)$, followed by vibrational relaxation to the zeroth level $(j=$ 0 ). The wave-function overlap of the states is indicated by the green area. Radiative transitions from the CT state to lower vibrational modes of the ground state are denoted by red arrows. c, $\Delta V_{n r}$ as a function of the $\mathrm{E}_{\mathrm{CT}}$ presents an empirical trend that a small $\mathrm{E}_{\mathrm{CT}}$ tends to yield a large non-radiative loss. (Reproduced with permission.[84] Copyright 2017, Springer Nature.)

with both narrow gaps and decent emissive efficiency, which indicates that the nonradiative loss is more complicated than what the energy-gap law can predict.

\subsubsection{Energetic offsets}

Early studies widely observed a correlation between $\mathrm{V}_{\mathrm{OC}}$ and the energetic difference between the HOMO of the donor and the LUMO of the acceptor [86, 87]. Later investigations on fullerene-based solar cells revealed the CT state as the origin of $\mathrm{V}_{\mathrm{OC}}$ [46]. The reason for a good correlation between the $\mathrm{V}_{\mathrm{OC}}$ and the $\mathrm{E}_{\mathrm{CT}}$, as discussed in the previous section, is that sub-gap absorption features dominate the radiative recombination $\left(\Delta V_{2}\right)$. As a result, increasing the LUMO of acceptor or decreasing the HOMO of donors have been the most followed pathways to increase the $\mathrm{V}_{\mathrm{OC}}$ [88-90]. But it is not until recently, when non-fullerene solar cells with small driving energies emerged, that the importance of the relationship between non-radiative loss and the energetic offsets was revealed.

Recent studies show that the energetic offsets between the CT states $\left(\mathrm{E}_{\mathrm{CT}}\right)$ and the local excited states $\left(E_{\mathrm{LE}}\right)$ of narrow gap materials have evident impacts on non-radiative voltage losses of organic solar cells (Figure 3.8) [49, 61, 91, 92]. D-A systems with small offsets tend to give strong emission and small voltage losses [69, 75, 93, 94]. For those D-A blends with small offsets, a distinct phenomenon is that the high-sensitive EQE and EL spectrum almost overlap between the blend and the narrow-gap pristinematerial devices. The absence of red-shifted CT absorption and emission bands are attributed to the strong interplayed LE-CT state [49, 92]. These interplayed LE-CT states, in form of thermal population and hybridization, are capable to significantly 

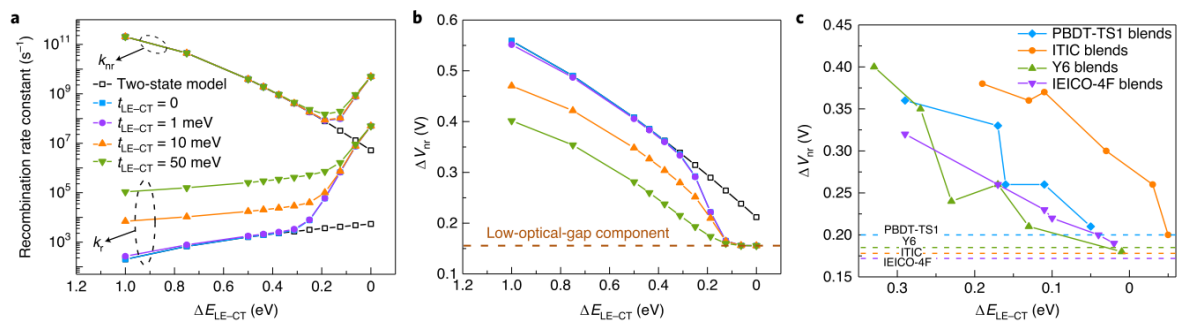

Figure 3.8 | Non-radiative voltage losses as a function of $\Delta \mathbf{E}_{\text {LE-CT. }}$ a, Radiative recombination rate $\left(k_{f}\right)$ and non-radiative recombination rate $\left(k_{n r}\right)$ at various electronic coupling between LE and CT states $\left(\mathrm{t}_{\mathrm{LE}-\mathrm{CT}}\right)$. b. Calculated non-radiative loss as a function of energetic offsets and electronic coupling. c, Measured non-radiative loss as a function of energetic offsets. (Reproduced with permission.[91] Copyright 2021, Springer Nature.)

enhance the emission efficiency through the way of oscillator strength borrowing from LE to CT states [91]. In addition, a few state-of-the-art organic solar cells with NIR absorption edge but without distinct CT optical transition have achieved reduced non-radiative losses and record efficiency, which marks the practical feasibility of this method [20, 95]. However, the energetic offsets cannot be reduced indefinitely. On the one hand, small energetic offsets hinder the charge separation and cause a low photo current [61]. On the other hand, the suppression of non-radiative loss seems to slow down with the proceeding of reducing energetic offsets [91].

\subsubsection{Other methods to reduce non-radiative losses}

A typical electroluminescence quantum efficiency can be expressed as

$$
E Q E_{E L}=f_{e-h} \beta \eta f_{\text {outcoupling }}
$$

where $f_{e-h}$ is the probability of balanced charge injection (when the numbers of electrons and holes injected are equal), $\beta$ is the probability of forming a correlated electronhole pair or exciton from each pair of injected carriers, $\eta$ is the emission quantum yield of the correlated electron-hole pair or exciton, and $f_{\text {outcoupling }}$ is the optical outcoupling coefficient. From this equation, some pathways for reducing non-radiative losses become apparent. Optimizing the injection barriers at both sides and the mobility balance, will ensure the recombination center within the active layer, make $f_{e-h}$ approach 1, and thus increase $E Q E_{E L}$. A device with good light outcoupling can also increase the emission efficiency. For a light-emitting diode with only one emissive species, $\beta$ usually denotes the ratio of singlets (typically $1 / 4$ ), and $\eta$ implies the photoluminescence quantum yield. Both $\beta$ and $\eta$ are expected to be increase for a high $E Q E_{E L}$. However, for blends where multiple kinds of excitons with different $\eta$ may compete with each other, Equation 3.8 shall be transformed to

$$
E Q E_{E L}=f_{e-h} f_{\text {outcoupling }} \sum \beta_{i} \eta_{i}
$$


where $\beta_{i}$ and $\eta_{i}$ correspond to the same type of exciton i. In a certain case, when $\eta_{i}$ and $\sum \beta_{i}$ are constant, increasing the portion of $\beta$ with higher $\eta$ can also increase the final $E Q E_{E L}$. In a blend film with negligible driving energy, the overlap of EL spectra between the blend and the pristine material implies that the radiative recombination in the blend is possibly determined by the emission properties of the pristine material with the lowest bandgap $[49,96]$. As also discussed in the previous subsection, the thermal population and electronic coupling have been reported as pathways to this phenomenon [91]. In other words, it also indicates the importance of enhancing the emission efficiency of the narrow-bandgap material to decrease the non-radiative recombination loss of the blend film. In a very popular system, PM6:Y6, it is also reported that the radiative recombination of free charges is almost entirely due to the re-occupation and decay of $\mathrm{Y} 6$ singlet excitons, but this pathway contributes less than $1 \%$ to the total recombination [97]. The paper claims that the properties of the Y6 singlet excitons are almost irrelevant for the $\mathrm{V}_{\mathrm{OC}}$ of the PM6:Y6 devices. Indeed, the total recombination is almost irrelevant with singlet excitons but is instead dominated by the CT state decay properties, which may give the impression that the decay rate change of singlets does not affect the total rate. However, if the singlet emission efficiency increases in EL, the total recombination rate including both radiative and non-radiative processes has to be reduced to ensure that the radiative recombination rate is constant according to the reciprocity principle, which leads to the a reduce $\mathrm{V}_{\mathrm{OC}}$ loss. Nevertheless, it is difficult to draw general conclusions, especially considering lacking adequate device examples. 



\section{CHAPTER 4}

\section{Charge dynamics and the fill factor}

This chapter reviews the basic processes of charge carrier generation in bulk heterojunction organic solar cells and discusses the loss channels behind the equilibrium. Parameters have been extracted from photophysical processes to describe the inherent properties of materials or devices. Finally, the fill factor in organic solar cells are discussed and linked to those parameters.

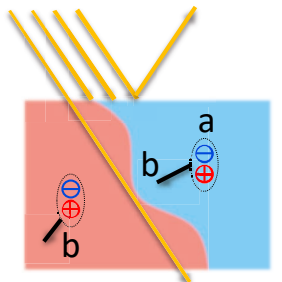

a. Exciton generation

b. Exciton diffusion

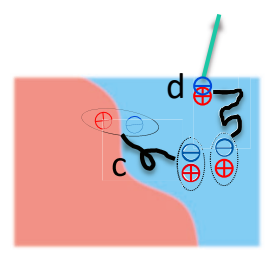

c. Charge transfer

d. Exciton recombination

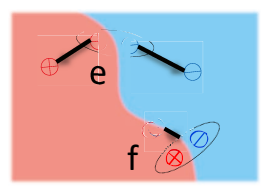

e. CT state separation

f. CT recombination

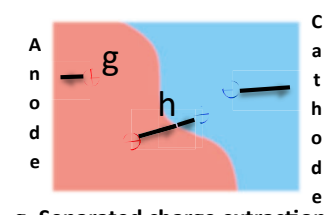

g. Separated charge extraction h. non-geminate recombination

Figure 4.1 Photons in and charges out. Schematic illustration of the photophysical processes in a bulk-heterojunction organic solar cell.

Generating photocurrent in a bulk-heterojunction organic solar cell requires several steps. Initially, photons are absorbed by the donor or the acceptor molecule, followed by exciton generation. The excitons undergo random walking, and they may diffuse toward the D-A interface, where a type of interfacial excited state is formed through ultrafast charge transfer. The interfacial state, which is called the CT state, is typically regarded as the precursor to free electrons and holes. The free charge carriers can drift and diffuse in the bulk materials, finally extracted at the electrodes. Excitons may recombine directly before they diffuse to the D-A interface, and CT states may also relax 
before forming charge-separated states. These steps are summarized in Figure 4.1. Note that, some photophysical processes in bulk-heterojunction organic solar cells are still controversial and need further understanding. The reason might be complex and diverse experimental phenomenon depending on the detailed material system and its specific micro-structures, while direct and effective observation is limited by disordered or unstable properties of organics.

\subsection{Charge-carrier photogeneration}

\subsubsection{Photon absorption and exciton generation}

In a D-A bulk heterojunction layer, both photoactive constituents can contribute to light absorption. However, despite tunable gaps, organic semiconductors tend to have a narrow absorption range compared to inorganic semiconductors. Fortunately, state-of-the-art organic photovoltaic materials with intramolecular electron pushpulling effects alleviate the problem by broadening the absorption range (over 300 $\mathrm{nm}$ ) yet maintaining strong extinction coefficients (over $10^{5} \mathrm{M}^{-1} \mathrm{~cm}^{-1}$ ) $[96,98]$. A D-A blend with complementary absorption can absorb most (over 80\%) of the light above the gap with only $130 \mathrm{~nm}$-thick film [20, 95].

Frenkel excitons form in the donor and acceptor molecules after light absorption. The excitons of organic material can relax to the ground state within hundreds of picoseconds without external influences $[15,61]$.

\subsubsection{Exciton diffusion}

Excitons diffuse within the condensed phase from one site to another via energy transfer before they decay to the ground state. The energy transfer can occur through Föster (through-space) or Dexter (through-bond). The exciton diffusion length in amorphous $\pi$-conjugated polymers covers a range of 5-15 $\mathrm{nm}$ [99-103]. while the latest study shows long exciton diffusion lengths in the range of 20 to $47 \mathrm{~nm}$ within non-fullerene acceptor films [71].

At D-A interfaces, the exciton from a donor unit dissociate via electron transfer from the donor to an acceptor unit and subsequently form CT excitons. A similar process can happen for an acceptor exciton via hole transfer. To ensure efficient charge transfer in a D-A blend, the donor or acceptor phase has to be small enough for excitons to reach the D-A interfaces before they decay, that is why the phase separation is so important for high device performance.

\subsubsection{Charge transfer}

In a molecular orbital picture, the driving force for charge transfer comes from the energy difference, also referred to as the energy offset, of either the LUMO of D and A in the case of electron transfer, and the HOMO in the case of the hole transfer. In condensed phases, the same must be true for the difference in electron affinity (EA) for electron transfer, and for the difference in ionization potential (IP) for hole transfer. 
Note that the EA and IP are often approximated by the LUMO and HOMO in molecular solids, ignoring the effects of intermolecular interactions. In an energy level picture, the driving force is the energy offset between the LE and the CT state. The driving force determines the favourable charge transfer direction from high energy to low energy, and also affects the transfer rate. Classical Marcus theory describe the electron transfer rate between individual molecules in solution as [104]

$$
W=\frac{J^{2}}{\hbar} \sqrt{\frac{\pi}{\lambda k T}} \exp \left[-\frac{\left(\lambda+\Delta G_{0}\right)^{2}}{4 \lambda k T}\right]
$$

where $J$ is the electronic coupling, $\lambda$ is the reorganization energy, $\Delta G_{0}$ denotes the difference in Gibbs free energy of two states, $\hbar$ is the reduced Planck constant, $k$ is the Boltzmann constant, and $\mathrm{T}$ is the temperature. If the tunneling between the initial and the final state needs to be taken into account, a more complete quantum mechanical treatment is required that incorporates both the effect of high and lowfrequency molecular modes, which is Marcus-Levich-Jortner expression [105].

$$
W=\frac{J^{2}}{\hbar} \sqrt{\frac{\pi}{\lambda_{0} k T}} \sum_{n=0}^{\infty} e^{-S} \frac{S^{n}}{n !} \exp \left[-\frac{\left(\lambda_{0}+\Delta G_{0}+n \hbar \omega\right)^{2}}{4 \lambda_{0} k T}\right]
$$

where $n$ is the quantum number of final vibrational state, $S$ is the Huang-Rhys factor, $\hbar \omega$ is the average vibrational energy spacing.

In fullerene solar cells, a number of studies using ultrafast pump-probe spectroscopy state that electron transfer occurs on a timescale of 10-100 fs, much faster than other photophysical processes, including singlet relaxation to the ground state (100 ps $1 \mathrm{~ns})[52,53,106,107]$. These studies denote a CT yield near $100 \%$ independent of the energy difference between $\mathrm{E}_{\mathrm{LE}}$ and $\mathrm{E}_{\mathrm{CT}}$. Some contradictory results are shown in time-resolved microwave conductivity and photoluminescence lifetime investigations on polymer-fullerene blends, where high CT rates only occur for an energetic offset of around $0.4 \mathrm{eV}[40,56]$.

Several reports on polymer:non-fullerene systems report a reduced CT rate on the picosecond time scale, attributed to the low driving force [108, 109]. A recent study demonstrates that hole transfer remains at hundreds femtoseconds with a negligible driving force [54]. The transfer rates are shown to be consistent with the prediction of Marcus theory. In addition, the study shows the electron transfer is generally faster $(<0.1 \mathrm{ps})$ which cannot be described within the non-adiabatic Marcus limit. Another study challenges previous understandings, showing that the ultrafast donor-toacceptor energy transfer precedes hole transfer from the acceptor to the donor and thus renders the EA offset virtually unimportant [62]. It also indicates the energy-level bending at the D-A interface is caused by the acceptors' quadrupole moments. This energy-level bending prevents efficient transfer from excitons to CT states at low IE offsets, while the same bending can facilitate the dissociation of CT excitons.

\subsubsection{Charge-pair dissociation}

After charge transfer, two scenarios are possible: either the electron and the hole are still weakly bound due to Coulombic interaction - or they are not and thus are free 

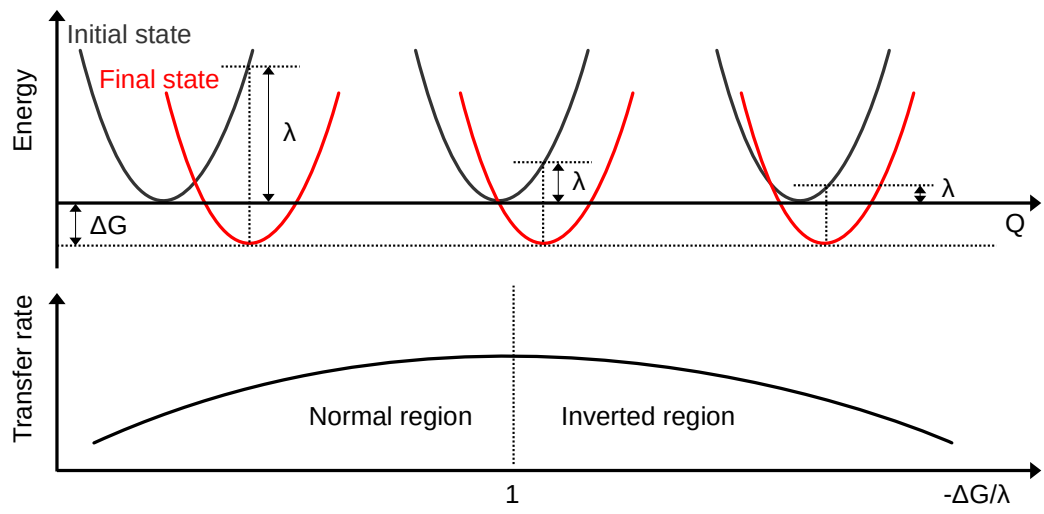

Figure 4.2 | Illustration of the Marcus theory for electron transfer. The potential diagram of a transition is shown from an initial state to a final state, mediated by a surrounding solvent. The donor molecule, the accetpor molecule, and the solvent are regarded as a system. The system is described by a generalized configuration coordinate $\mathrm{Q}$ and the related potential energy curve that is approximately a parabola. $\Delta G_{0}$ is the Gibbs free energy difference between the initial and finial states, and $\lambda$ is the reorganization energy describing the energy required to match the geometries of the final and initial states. Credit: Dr. Jiangbin Zhang.

carriers. If CT excitons are generated upon singlet exciton dissociation, an additional driving force is needed to overcome the Coulomb binding energy.

The Braun-Onsager model is widely used to describe the field-dependent dissociation of charge pairs,

$$
P(F)=\frac{k_{\text {diss }}(F)}{k_{d i s s}(F)+k_{f}}
$$

where $P(F)$ is the dissociation yield of electrical field $F, k_{d i s s}(F)$ is the rate of dissociation with $F$, and $k_{f}$ is the recombination recombination rate to the ground state. The field-dependent dissociation rate $k_{d i s s}$ is expresses as

$$
k_{d i s s}(F)=\frac{e \mu}{\varepsilon_{r} \varepsilon_{0}} \frac{3}{4 \pi a^{3}} \exp \left(-\frac{E_{b}}{k_{B} T}\right)\left(1+b+\frac{b^{2}}{3}+\frac{b^{3}}{18}+\cdots\right)
$$

where $\mu$ is the sum of electron and hole mobility, a is the initial distance of its constituents, $E_{b}=e^{2} / 4 \pi \varepsilon_{r} \varepsilon_{0} a$ is the binding energy, $k_{B}$ is the Boltzmann constant, $\mathrm{T}$ is the temperature, and $\varepsilon_{r} \varepsilon_{0}$ is the dielectric constant. The last factor in brackets is the Taylor expansion of a first-order Bessel function, with the reduced electric field $b=e^{3} F / 8 \pi \varepsilon_{r} \varepsilon_{0}\left(k_{B} T\right)^{2}$.

Despite the binding energy of tens to hundreds of meV [43, 110-112], the CT state dissociation yield has been shown to approach $100 \%$ even when directly exciting the 
vibrationally relaxed CT state [113]. There have been many explanations for efficient charge generation. High local mobility in crystalline PCBM clusters was proposed to facilitate dissociation by performing a Braun-Onsager fit in polymer fullerene films [114]. Another study indicates that the Coulomb binding energy of the CT can be reduced by electrostatic screening and delocalization of charges, enabling separation from thermally relaxed CT states [115]. Moreover, the importance of delocalization and the crystallinity of the acceptor phase for efficient separation has been suggested by others as well [116-119]. It has also been suggested that CT separation is entropydriven [120, 121]. The density of states is drastically increased for free charges as compared to bound charges at the interface, leading to an increase in entropy and making charge separation energetically favourable. Recent studies show barrierless CT state to free charge conversion in high-performance non-fullerene acceptor solar cells, and the origin has been revealed to be the energy level bending at D-A interface caused by quadrupole moments of the acceptors $[62,72]$.

\subsubsection{Charge-carrier transport}

To be collected by electrodes, free electrons and holes have to transport within condensed phases. As mentioned in subsection 2.1.2, charge motion can be driven by an electric field (drift) or a charge-concentration gradient (diffusion). A key parameter that describes the motion ability is mobility $\mu$, which is defined as the drift velocity $v$ per unit electric field $F$

$$
\mu=\frac{v}{F}
$$

Note that, $\mu$ is not necessarily a constant and it may change along with the electric field. [122-124].

In an organic semiconductor, charge transport is accomplished by the transfer of an electron from the LUMO of a site to the empty LUMO of another site. Equivalently, a hole can be transferred between HOMO levels of sites. Here, the site may be a molecule or a conjugated segment of a polymer. In an ordered crystal, carriers can move within a band of states formed by the frontier orbitals. The acceleration under an electric field ends at a finite velocity due to the increasing scattering along with the increasing velocity. This is the reason for a finite mobility. As long as this scattering process is only a weak perturbation of the overall electronic coupling between the neighboring molecules, charge transport can be described by the bandtransport model [125]. In this model, a mobility decreases with increasing temperature due to increasing scattering. At higher temperatures, intra-molecular vibrations also become important. This can be described by the local phonon coupling, where the "phonon" is molecular vibrations and the "coupling" is of the vibronic type [126]. If the strength of vibronic coupling is at the same level as that of electronic coupling between sites, a band-transport model is no longer suitable for describing the charge transport process. In the extreme case, the transport becomes a hopping-like motion when the charge motion comes across scattering at every site [127, 128]. Furthermore, when the material is microscopically disordered, transport is controlled by (i) the electronic coupling among the constituent molecular units, (ii) the coupling to intra-molecular as well as inter-molecular vibrations, and (iii) the static intra- and 
Ch. 4 | Charge dynamics and the fill factor

inter-molecular disorder [15].

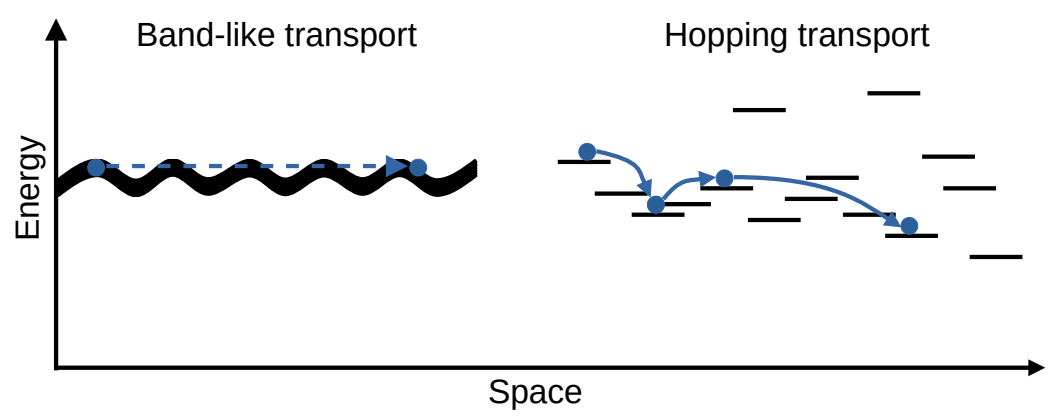

Figure 4.3 | The band-like transport and the hopping charge transport. For the band-like transport, electronic coupling between molecules is dominating, and the mobility decreases with increasing temperature. For the hopping transport, thermally-assisted tunneling is dominating, and the mobility increases with increasing temperature. Credit: Dr. Nakul Jain.

The charge transport mode in organic solar cells is disorder controlled due to the amorphous nature of the organic blend films, despite some crystalline or semi-crystalline domains. A commonly used model to explain charge carrier transport in disordered organic solids on a microscopic level is the Bässler model, also called the Gaussian disorder model (GDM) [129]. In this model, the DOS can be described by a Gaussian distribution as discussed in Equation 2.9. This approach assumes that the energies of adjacent sites are uncorrelated, which is not necessarily true. The jump rate $v$ from site $i$ with energy $E_{i}$ to $j$ with $E_{j}$ is assumed to be of Miller-Abrahams type [130],

$$
v_{i j}=v_{0} \exp \left(-2 \gamma r_{i j}\right) \begin{cases}\exp \left(-\frac{E_{j}-E_{i}}{k_{B} T}\right), & E_{j}>E_{i} \\ 1, & E_{j} \leq E_{i}\end{cases}
$$

where the first exponential term of this equation describes the wave-function overlap and a tunneling probability, $r_{i j}$ is the jump distance between site $\mathrm{i}$ and site $\mathrm{j}, \gamma$ is the inverse localization radius which is a wave-function decay parameter, $v_{0}$ is the attemp-to-hop frequency.

The Miller-Abrahams equation neglects the polaronic effect as described by Marcus theory, such as changes in molecular conformation upon charge transfer. The hopping rates given by both theories are typically applied in Monte Carlo simulations [129, $131,132]$ or hopping master equations $[133,134]$ to study the macroscopic charge carrier transport properties in organic disordered semiconductors. 


\subsection{Geminate and non-geminate recombination}

\subsubsection{Recombination of geminate pairs}

The recombination of a positive charge and a negative charge may occur geminately or non-geminately. In organic solar cells, geminate recombination (GR) is closely related to the free charge generation, while non-geminate recombination (NGR) mainly competes with charge extraction.

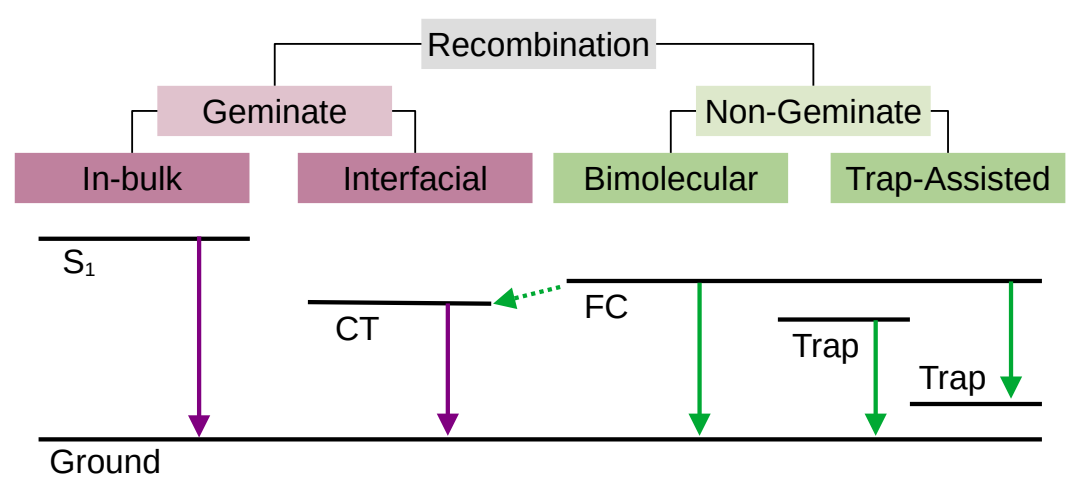

Figure 4.4 | Geminate and non-geminate recombination in an energy diagram. Singlets and CT excitons recombine geminatey by relaxation directly to the ground state. Free charge (FC) carriers recombine non-geminately through back-transfer, bimolecular and trap-assisted processes. Credit: Dr. Nakul Jain.

Originally, the term "geminate recombination" refers to the reaction of two transient species produced from a common precursor in solution [135]. The pair of species from a common precursor is called a geminate pair. If a recombination reaction occurs before any separation of the geminate pair due to diffusion, this reaction is termed primary GR. If the interacting particles have been separated and come together through diffusion, this reaction is termed secondary GR. In organic heterojunctions, electron-hole pairs which are still correlated due to their mutual Coulomb attraction, are also called geminate pairs. The recombination of such an electronhole pair is called GR as well. Therefore, a geminate pair in organic solar cells comes from the same parent exciton. If positive and negative charges are injected at the anode and the cathode, subsequently move through the organic semiconductor film, encounter each other, and finally relax to the ground state or form a geminate pair, then this is a NGR process. Similarly, photogenerated free charge carriers undergo NGR as well.

GR and NGR are not two competing processes. Instead, they are describing the spontaneous recombination processes of geminate pairs and free charge carriers, respectively. A geminate pair undergoes GR spontaneously, or dissociates via a driving force. A charge carrier undergoes NGR spontaneously, or gets extracted (recombining elsewhere). Moreover, geminate pairs can also come from NGR in addition to the same 
excitation. This is because GR includes secondary GR in its definition. In addition, there is no proof that a geminate pair can discriminate its origin and recombine differently. Accordingly, NGR should not be considered as complete recombination process, to avoid the description of the later process and the argument whether it is a relaxation to the ground state or the source of geminate pairs.

\subsubsection{Recombination of free carriers}

Photogenerated or injected free charge carriers can meet each other and recombine non-geminately during the charge transport. Langevin theory is often used to describe the charge recombination in materials with low charge mobility $\left(\mu<1 \mathrm{~cm}^{2} /(V\right.$. s)) [136]. This theory was developed by Langevin in 1903 to describe recombination of ions in gases [15, 137].

$$
R=-\frac{d n}{d t}=-\frac{d p}{d t}=-\gamma n p
$$

where $R$ is the recombination rate per unit volume, $n$ and $p$ are the concentration of electrons and holes, and $t$ is time. The recombination coefficient, $\gamma$, is the product of the charge-moving velocity and reaction cross-section. If $n=p, R \propto n^{2}$. Since this recombination involves two molecules and the recombination order is two, the recombination process is called bimolecular recombination (BMR). This model assumes a recombination critical point where the Coulomb potential euqals the thermal energy $k_{B} T$, which can be expressed as

$$
r_{c}=\frac{e^{2}}{4 \pi \varepsilon_{r} \varepsilon_{0} k_{B} T}
$$

where $r_{c}$ is known as the Coulomb capture radius, as the Langevin capture radius or as the Onsager radius. With a collision sphere of this radius, the recombination coefficent can be described by

$$
\gamma=4 \pi r_{c}\left(D_{n}+D_{p}\right)
$$

where diffusion coefficients of positive and negative charges are expressed as $D_{n}$ and $D_{p}$, respectively. Using Einstein relation $D=\mu k_{B} T / e$ and Equation 4.8, Equation 4.9 becomes

$$
\gamma=\frac{e}{\varepsilon_{r} \varepsilon_{0}}\left(\mu_{n}+\mu_{p}\right)
$$

where $e$ is the elementry charge, $\varepsilon_{r}$ is the relative permittivity, $\varepsilon_{r}$ is the vacuum permittivity, and $\mu_{n}$ and $\mu_{p}$ are mobilities of negative and positive charges, respectively. However, Langevin recombination theory is often found to deviate from experimental results, espeically for the recombination coefficients of efficient organic solar cells [138141]. Recombination coefficients, that are one to three orders of magnitude lower than the prediction of Langevin theory, have been reported for both fullerene and non-fullerene organic solar cells [111, 142-145]. Therefore, a reduced Langevin recombination coefficient is introduced. One possible reason for reduced recombination probability is that electrons and holes stay at donors and acceptors respectively, and the recombination can take place only at the D-A interfaces [138]. Interestingly, 
the reduction factor has not been explained comprehensively due to a strong dependence on the material system and its processing condition [146].

For several material systems, a recombination order above two has been observed [147, 148]. Although recombination of order 3 is often considered as Auger recombination, it is less possible in organic solar cells. Because AM 1.5G illumination is commonly not enough to provide the high charge carrier densities for Auger recombination. The high recombination order is usually explained by effects depending on the chargecarrier density, for example, density-dependent mobilities or recombination coefficients

\subsection{The fill factor}

The definition of a fill factor is shown in Equation 2.6. A fill factor (FF) measures whether a solar cell can output a current and a voltage approximating its conversion limit simultaneously. Note that, for a large-scale solar cell, the series resistance significantly limits the FF, which is not discussed in this work. Physically, the FF is resulting from the competition between charge recombination and charge extraction as a function of the voltage bias. The following subsections introduces how these processes are affecting the FF of solar cells, especially organic solar cells.

\subsubsection{Analytical expressions}

In Figure 3.3, we show the numerical expression of maximum FFs. In this subsection, we discuss the analytical expressions for FFs, as well as impacts from recombination coefficient, mobilities, and thickness.

Independent of the relationship between an current output $J$ and a voltage output $V$, the condition for maximum power yields

$$
d(J V)=J d V+V d J=0
$$

and thus

$$
\left(\frac{d J}{d V}\right)_{M P P}=-\left(\frac{J}{V}\right)_{M P P}
$$

where MMP represents the maximum power point. An ideal exponetial J-V characteristic is expressed as

$$
J=J_{0}\left(\exp \left(\frac{e V}{n_{i d} k_{B} T}\right)-1\right)+J_{S C}
$$

where $n_{i d}$ represents the ideal factor, $J_{0}$ the reverse saturation current, $e$ the elementry charge, $k_{B}$ the Boltzmann constant, T the temperature, and $J_{S C}$ the short-circuit current. With Equation 4.13, Equation 4.12 becomes

$$
J_{0} \frac{e}{n_{i d} k_{B} T} \exp \left(\frac{e V_{M P P}}{k_{B} T}\right)=-\frac{J_{M P P}}{V_{M P P}}
$$


Ch. 4 | Charge dynamics and the fill factor

With

$$
J_{M P P}=J_{0}\left(\exp \left(\frac{e V_{M P P}}{n_{i d} k_{B} T}\right)-1\right)+J_{S C}
$$

and

$$
\frac{J_{S C}}{J_{0}}=1-\exp \left(\frac{e V_{O C}}{k_{B} T}\right)
$$

Equation 4.14 becomes

$$
V_{M P P}=\frac{n_{i d} k_{B} T}{e}\left[\exp \left(\frac{e\left(V_{O C}-V_{M P P}\right)}{n_{i d} k_{B} T}\right)-1\right]
$$

If we divide the voltage by $n_{i d} k_{B} T / e$ and use the symbol $v$ for this normalized voltage, we get

$$
v_{M P P}=v_{O C}-\ln \left(v_{M P P}+1\right)
$$

which leads to a precise implicit relationship for FF

$$
F F=\frac{v_{M P P}}{v_{M P P}+1} \frac{v_{O C}-\ln \left(v_{M P P}+1\right)}{v_{O C}\left(1-\exp \left(-v_{O C}\right)\right)}
$$

If we do a first approximation, replacing $v_{M P P}$ with $v_{O C}$ and neglecting $\exp \left(-v_{O C}\right)$

$$
F F=\frac{v_{O C}-\ln \left(v_{O C}+a\right)}{v_{O C}+1}
$$

where $a=1$. Emperially, setting $a=0.72$ greatly increases the accuracy of the expression, as proposed by Green [149]. This equation is derived from the Shockley equation and only valid if the $V_{M P P}$ is close to the $V_{O C}$.

\subsubsection{Figure of merits for organic solar cells}

A figure of merit is a single quality parameter extracted from a multifactorial system or a device to describe its performance. A FF itself is a figure of merit of a solar cell. However, it is difficult to analytically relate the fill factor to the mobility, the film thickness, and the recombination coefficient. From the perspective of those parameters, new figure of merits (FOMs) have been proposed to describe the device performance, as well as the FF of organic solar cells.

In organic solar cells, a low mobility (typically below $10^{-2} \mathrm{~cm}^{2} / \mathrm{Vs}$ ) severely limits charge extraction and causes considerable pile-up of photogenerated charges in the active layer [150]. The pile-up results in significant NGR and screens the electric field from the externally applied bias, which leads to non-ideal JV characteristics. A dimensionless parameter $\theta$ has been proposed under consideration of both recombination and extraction [151]

$$
\theta=\frac{k_{2} G d^{4}}{\mu_{n} \mu_{p}\left(V_{i}\right)^{2}} \propto \frac{J_{r e c}}{J_{\text {extr }}}
$$

where $k_{2}$ is the second order NGR coefficient, $G$ is the generation rate, $d$ is the active layer thickness, $\mu_{n}$ and $\mu_{p}$ are the electron and hole mobilities, $V_{i}$ is the internal bias 
(here taking the electrode work function difference minus $0.4 \mathrm{~V}$ ), and $J_{\text {rec }}$ and $J_{\text {extr }}$. A clear dependence of fill factors on $\theta$ is shown in Figure 4.5 with little scatter, by varying $\theta$-related parameters to simulate the JV characteristics.

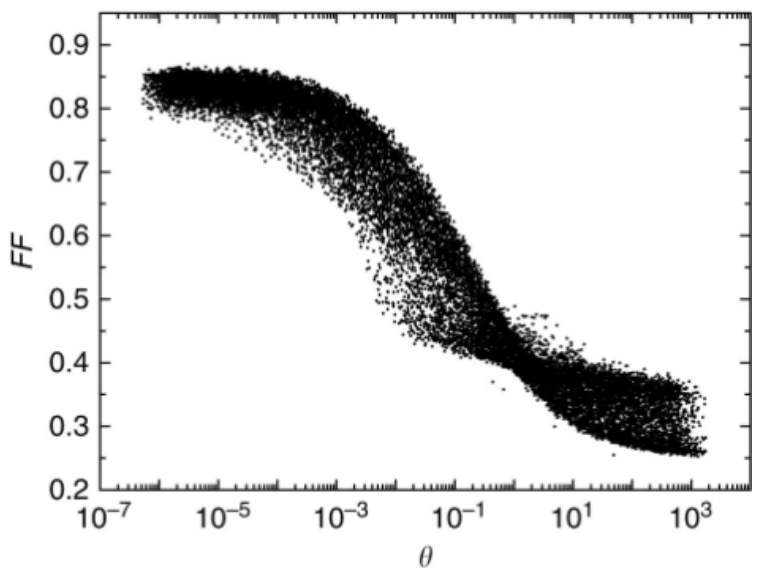

Figure 4.5 Simulated FF- $\boldsymbol{\theta}$. Scattered points show a clear "S" shape, which indicates the correlation between two parameters. Reproduced under a Creative Commons Attribution 4.0 International License.[152]

Another dimensionless parameter $\alpha[150]$ is defined as

$$
\alpha^{2}=\frac{e^{2} k_{2} J_{G} d^{4}}{4 \mu_{n} \mu_{p}\left(k_{B} T\right)^{2}}
$$

where $e$ is the elementry charge, and $k_{B} T$ is the thermal energy. An approximate value for $V_{i n t}$ is obtained by assuming constant gradients of the quasi Fermi levels across the device. This parameter omits the impacts from the electrodes and transport layers, but focuses on the bulk recombination within the active layer, which describes the electronic quality of a photovoltaic layer. To get an analytical expression, which relates a FF to the parameter $\alpha$, an equation similar to Equation 4.20 is needed. Considering that the Shockley equation is imcomplete to describe an organic solar cell, the following empirical expression is used to describe the FF

$$
F F=\frac{v_{O C}-\ln \left(0.79+0.66 v_{O C}^{1.2}\right)}{v_{O C}+1}
$$

with $v_{O C}$ written as

$$
v_{O C}=\frac{e V_{O C}}{(1+\alpha) k_{B} T}
$$

to provide a viable analytical description of the FF of solar cells with transport-limited photocurrents [150].

As shown in Figure 4.6, the simulation results in the FF versus $\alpha$ plots, which include a wide range of mobilities, recombination coefficients, and thicknesses, have small 
Ch. 4 | Charge dynamics and the fill factor

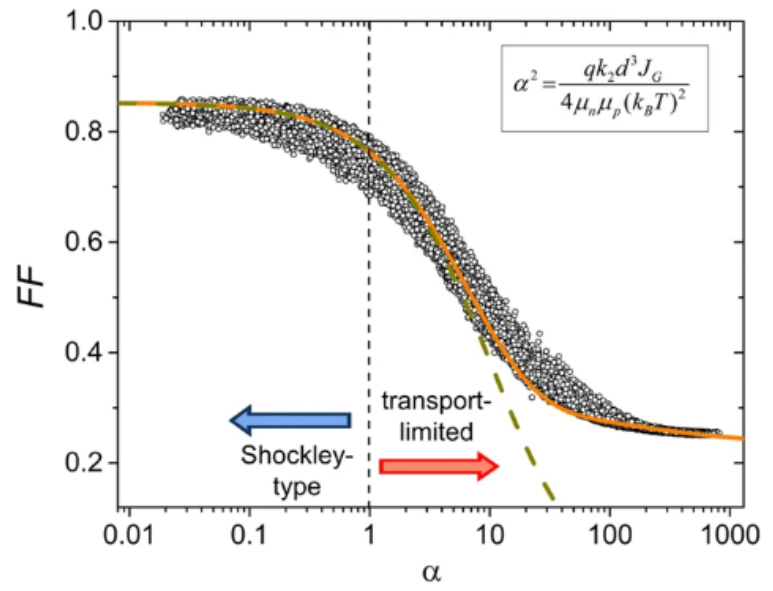

Figure 4.6 | Illustration of the new figure of merits. Open circles are simulated FF- $\alpha$ points. Dashed and solid lines show analytical FF- $\alpha$ dependencies according to Equation 4.20 and Equation 4.23. Reproduced under a Creative Commons Attribution 4.0 International License.[150]

scatter with the analytical expression curve. Note that the simple expression Equation 4.20 only works well for the ideal behavior $(\alpha<1)$, while Equation 4.23 provides a much better description of the full data, especially the transport-limited region $(\alpha$ $>1)$.

Another study presents a facile method to extract the photoactive layer's electronic quality by using only common device parameters [153]. The FF is normalized to reduce the scatter shown in the previous method and the simulation results, by mapping the FF onto the range $(-1,1)$

$$
F F_{n}=2 \frac{F F-F F_{\min }}{F F_{\max }-F F_{\min }}-1
$$

where $F F_{\min }$ is set 0.25 , corresponding to non-rectifying device with a linear JV curve, and $F F_{\max }$ a simple relation proposed in an early work

$$
F F_{\text {max }}=\frac{a V_{O C}}{V_{O C}+b}
$$

where $\mathrm{a}$ and $\mathrm{b}$ are constant parameters, 0.92 and 0.09 , respectively, to be found by fitting. By assuming that $F F_{n}$ follows hyperbolic tangent function due to the $\mathrm{S}$ shape of scattered data points, authors did fitting with this equation

$$
F F_{n, f i t}(\gamma)=\tanh \left[\alpha \ln \left(\gamma / \gamma_{0}\right)\right]
$$

where $\alpha$ and $\gamma_{0}$ are fit parameters as well. $\gamma$ is the collection coefficient

$$
\gamma=\frac{V_{O C}^{2}}{d^{3.5} J_{S C}^{0.5}} \frac{\mu^{2}}{k^{0.8}}
$$


where $\mu$ is the mobility when balanced mobilities are assumed, and $k$ is the recombination coefficent. After fitting, they obtained $\gamma$, equivalently the electronic quality $\mu^{2} / k^{0.8}$ for a direct recombination (to be distinguished with Shockley-Read-Hall recombination)

$$
Q_{f i t}=\gamma_{0} \frac{d_{3.5} J_{S C}^{0.5}}{V_{O C}^{2}} \exp \left(\frac{\operatorname{arctanh} F F_{n}}{\alpha}\right)
$$

where $\gamma_{0}$ is $2 \times 10^{17} \mathrm{~mA}^{-0.5} \mathrm{~s}^{-1.2} \mathrm{~cm}^{-0.9}$, and $\alpha$ is 0.29 . This method is facile because it can get the competition between extraction and recombination by measuring only the device thickness and the JV curve. But the mathematical processing, including the normalization of FFs and the selection of hyperbolic tangent function for fitting, lacks physical meaning, which is also reflected in the irregular dimension of Q.
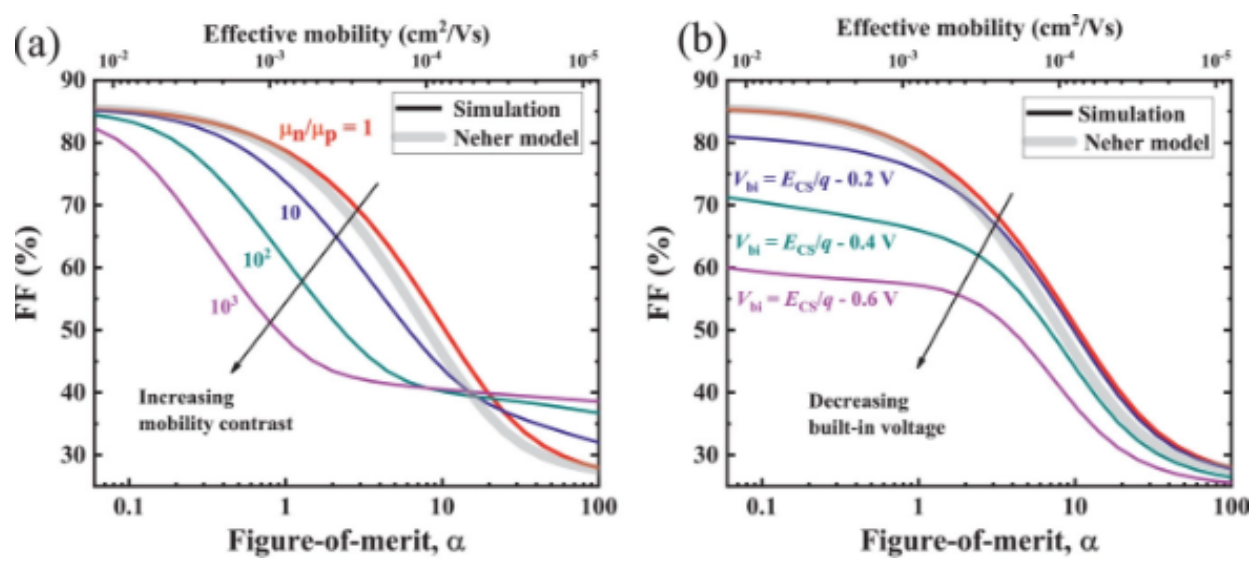

Figure 4.7 | Mobility imbalance and contact barriers. Simulated FFs shown in terms of the Figure-of-merit $\alpha$ to illustrate (a) the influence of a mobility imbalance and (b) the effect of increasing electrode work function mismatch at the contacts (decreasing built-in voltage). The FF expected based on the model of Neher et al.[150], is indicated by the grey line. (Reproduced with permission. [154] Copyright 2021, WileyVCH GmbH.

All the analytical analysis above assumes that i) space charge effects are insignificant, and ii) the bimolecular process dominates the recombination, while surface recombination and trap-assisted recombination are negligible. However, when mobilities of electron and hole are not balanced, the accumulation of slow charge results in space charge effects, causing additional NGR. This mobility mismatch has been widely blamed for non-optimal FFs [155-158]. As shown in Figure 4.7 a, a simulation result of several mobility ratios is compared with the model (Figure 4.6) in a recent review paper [154]. Another recombination pathway, considerable in organic solar cells, is surface recombination. The origin can be the reduction of the built-in field caused by inappropriate contact workfunction, surface pinning effects, and surface traps. The effect of built-in voltage on the FF is demonstrated in Figure $4.7 \mathbf{b}$.

In general, the models above can predict the impacts from mobility, recombination rate, and thickness on the fill factor well. However, major deviation occurs when the 
charge generation is dominated by the external voltage bias, which might be a problem in organic solar cells with a negligible driving force.

\subsubsection{Development of the fill factor in organic solar cells}

Looking back at organic solar cells, the FF did not rise steadily with the record efficiency. In 2013, the device based on PTPD3T:PC ${ }_{71} \mathrm{BM}$ achieved a 79.6\% FF with EQE close to $80 \%$ and PCE near $8 \%$ [159]. This is very impressive compared to MDMOPPV:PC ${ }_{61} \mathrm{BM}$ (61\% for FF and 2.5\% for PCE) and P3HT:PC ${ }_{61} \mathrm{BM}(67.4 \%$ for $\mathrm{FF}$ and $4.4 \%$ for PCE) [160, 161]. Fullerene solar cells reported with PCEs around 10\% did not show improvements in the FF values [162-164]. Later, the record efficiency gradually moved to non-fullerene. In 2016, the benchmark non-fullerene device based on PBDB-T:ITIC obtained FF 71\%-74\%, PCE close to $11 \%$ and EQE around 75\% [165]. The device based on PM6:Y6 has FF around 75\%, PCE above 15\% and EQE above $80 \%$ [166]. For the state-of-the-art non-fullerene solar cell with efficiency approaching $20 \%$, most of them have a FF around $80 \%$ [19, 20, 167]. Two decades ago, the low FF was due to limited material choices, insufficient understanding of mechanisms, or lacking device fabrication experience. During the age of fullerene, when great progress has been made in all aspects of organic solar cells, high FF and high EQE have already been achieved simultaneously. These achievements are attributed to the high electronic qualities of the active layer and the prolonged device optimization. However, the organic solar cell has a feature that significantly distinguishes it from silicon solar cells and perovskite solar cells, that its development relies heavily on the renewal of the main constituent materials. As a result, although the device physics and fabrication experience can be sustained and developed steadily, material and devices properties may show fluctuations. These fluctuations create difficulties for phenomenon understanding, but they also bring the ground for discovering general mechanisms and deep science.

Recently, it is reported the FF of organic solar cells decreases when the energetic offset goes down [168]. This raises the question that is there a relation between the FF and the driving energy. If there is, how are they related? Furthermore, this phenomenon is often related to a voltage-loss change, posting another question whether there is a relation between the FF and the voltage loss. These questions remain to be answered. 
The fill factor | Sec. 4.3

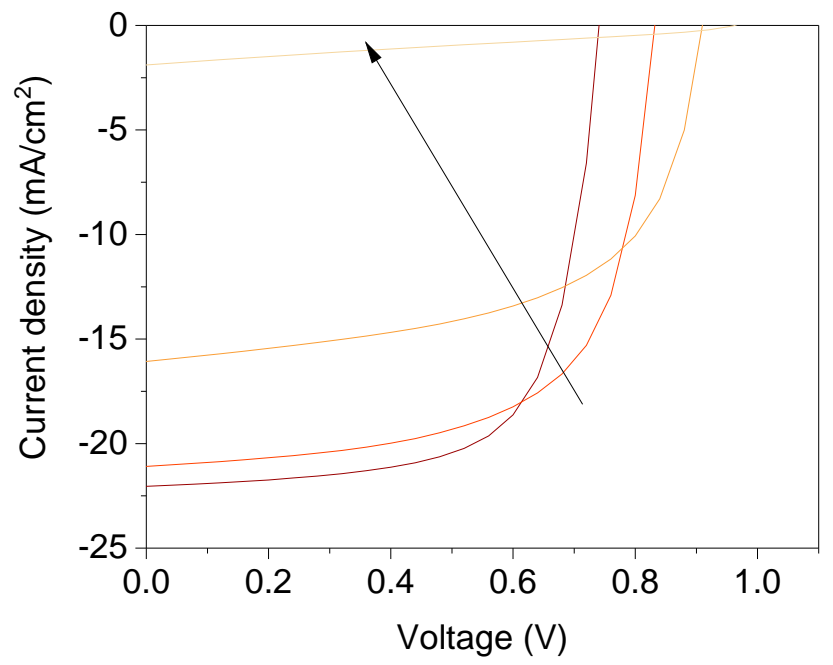

Figure 4.8 | Schematic for decreasing fill factors and decreasing voltage losses with decreasing energetic offsets. 



\section{CHAPTER 5}

\section{Summary and Outlook}

The core of this thesis is to understand the loss mechanisms of organic solar cells. To cope with this challenge, the thesis understands an organic solar cell from the perspective of thermal dynamics and equilibrium states, and the perspective of charge dynamics and intermedium states. Based on these two aspects, the open-circuit voltage and the fill factor in organic solar cells are analyzed.

The detailed balance in a solar cell works perfectly for the voltage loss analysis. To further relate the voltage loss to material properties, however, more internal and microscopic parameters of molecules and condensed phases should be taken into consideration. Currently, the voltage loss of the best organic solar cell is still greater than that of the best perovskite solar cell. To continue to improve the power conversion efficiency of organic solar cells, more efforts are needed to reduce the voltage loss, especially the non-radiative voltage loss. Compared with the open-circuit voltage, the fill factor lacks physical meaning and is more complicated. The process of charge generation, recombination, and transport, as well as their field dependence, have an impact on the fill factor. Thanks to previous studies on analytical expressions and new figure of merits, the fill factor in organic solar cells has been well described with assumptions. However, the assumptions limit their usage, and there are exceptional cases and trends that require additional investigation. Besides the voltage and the fill factor, the photocurrent, precisely the charge separation process, in organic solar cells is not entirely clear. Particularly, there are still debates on the energetic offsets, interfacial dipoles, interfacial electrostatic potentials, and the charge-transfer excitons.

Organic solar cells have been developing for over three decades. Achievements are in various fields, including material synthesis, mechanism understanding, characterization techniques, and device fabrication. With the efficiency approaching $20 \%$, the commercialization of organic solar cells is on the way. Therefore, several points should be considered in moving towards commercialization. Firstly, long-term sta- 
Ch. 5 | Summary and Outlook

bility, especially stability under light, is essential for organic solar cells. There is a need for in-depth understandings of degradation and stability in high-performing non-fullerene acceptors for future commercialization. Considering the unstable nature of organics under sunlight, we may use organic solar cells in weak light situations (for example, indoor) or for short-term use. Secondly, the use of green solvents is necessary for the large-scale commercialization of organic solar cells. Although unprecedented efficiency of over $16 \%$ in green-solvent processed non-fullerene organic solar cells has been achieved [169], there is a dearth of studies aimed at understanding the detailed morphology and device physics in these systems. Thirdly, some powerful tools such as the high-output automatic screening and machine learning in molecule design may accelerate the development. Although current reports are still rare, with the rapid development of artificial intelligence, the two fields are likely to collide and generate new sparks. 


\section{CHAPTER 6}

Methods

This chapter introduces the major experimental methods and equipments in this work.

\subsection{Sensitive-external-quantum-efficiency characteri- sation}

\subsubsection{Monochromatic technique}

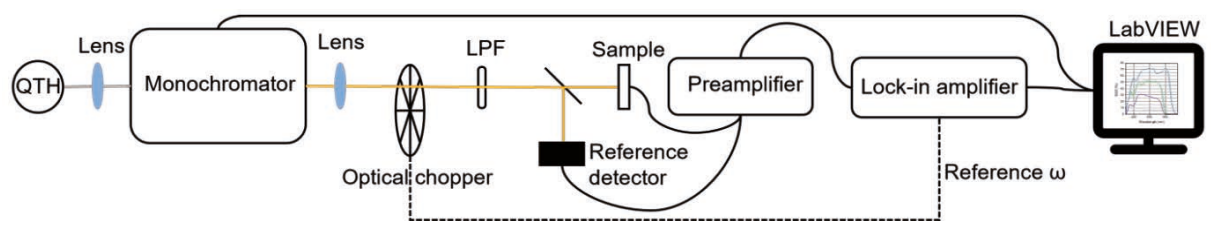

Figure 6.1 | Schematic illustration of the $E Q E_{P V}$ measurement through the monochromatic and the lock-in technique.

The definition of EQE is described in Equation 2.8. To experimentally measure the photocurrent at each wavelength, particularly the weak response of the absorption tail, researchers often use the lock-in technique to increase the signal-to-noise ratio. As shown in Figure 6.1, the light from a quartz tungsten halogen (QTH) lamp is focused into a monochromator (Bentham TMC300). A monochromator is an optical device that selects a specific narrow band of wavelengths of light from the input light. The output monochromatic light is modulated with an optical chopper (Thorlabs MC2000B) with frequency $\omega(165 \mathrm{~Hz}$ in my experiments). The frequency should aviod common AC signals and their integer multiples. Then the light passes through 
a long-pass filter (LPF), subsequently a beamsplitter. A part of light shines upon the device to generate photocurrents as the sample signal, while the other part of light goes to a silicon detector for calibration. The electrical signals from both the sample and the detector are received by a preamplifier (SR570) and subsequently a lock-in amplifier (SR830). The lock-in amplifier multiplies the sample signal by the reference signal $\omega$, integrates it over a specified time, and outputs a DC signal which is proportional to the amplitude of only $\omega$ in the sample signal, excluding noises of other frequencies. In our EQE measurements, the DC signals which are proportional to the photocurrent amplitudes are transferred to a homemade LABVIEW program. Using the $\mathrm{EQE}$ of the silicon detector and the light intensity ratio between the detector and the sample, the EQE of the sample can be calculated.

The working mechanism of a monochromator with the common Czerny-Turner design is shown in Figure 6.2. The incident light (A) is aimed at an entrance slit (B). The slit is placed at the effective focus of a concave cylindrical mirror (C) to collimate the reflection light. The collimated light is diffracted by a grating (D) and dispersed according to the color. The diffraction light is collected by another concave cylindrical mirror (E), which focuses the dispersed light on the plane of an exit slit (F). The exit slit with a finite width outputs the dispersed light selectively. A rotation of the grating changes the incident and diffraction angle, to project the desired wavelength onto the center of the exit slit.

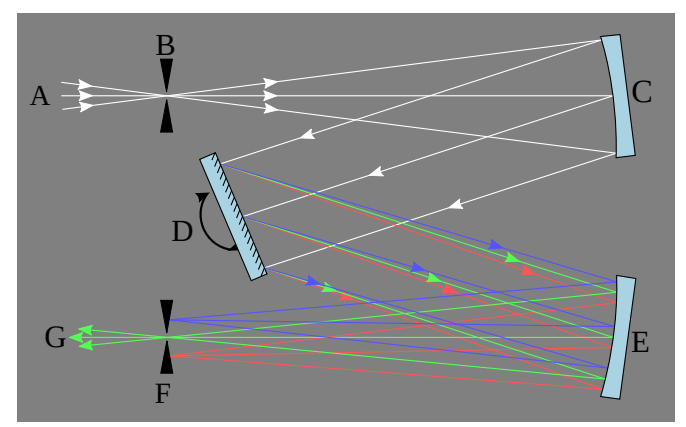

Figure 6.2 | Schematic illustration of a Czerny-Turner monochromator. The widerange light (A) is focused at an entrance slit (B), and subsequently collimated by a cylindrical mirror (C). The collimated beam is diffracted from a grating (D). The dispersed light is focused by another cylindrical mirror (E) onto the plane of the exit slit (F). Each wavelength of light is focused at a different position, and only the desirable range $(\mathrm{G})$ can pass through the exit slit by rotating the grating and adjusting the slit. Figures by FeuRenard under CC-BY-SA 4.0 license [170]

The basic grating equation determines the discrete directions into which monochromatic light of wavelength $\lambda$ is diffracted, which is expresses as

$$
m \lambda=d_{G}\left(\sin \alpha+\sin \beta_{m}\right)
$$

where $\alpha$ is incident angle, $\mathrm{d}_{G}$ is the groove spacing of the grating, and $\beta_{m}$ is the set of 
angles the light diffracted along. For every beam of monochromatic light incident on a grating, the light is diffracted in directions corresponding to $\mathrm{m}=0, \pm 1, \pm 2, \pm 3, \ldots$. Typically only the first order, positive or negative, is desired. However, the first-order diffraction of a specified frequency is in the same direction as the higher-order diffraction of its multiple frequencies. As a result, higher order diffraction peaks may need to be blocked. It is especially important to block high-order radiation when measuring the tail-state absorption. For example, if a solar cell has EQE of 1 at $500 \mathrm{~nm}$ and EQE of $10^{-5}$ at $1000 \mathrm{~nm}$, the second-order diffraction of $500 \mathrm{~nm}$ needs to be 6 orders of magnitude weaker than the first-order diffraction of $1000 \mathrm{~nm}$, to ensure the system error less than $10 \%$. This is why an additional LPF is often needed for the measurement of sub-gap features.

\subsubsection{Fourier-transform technique}

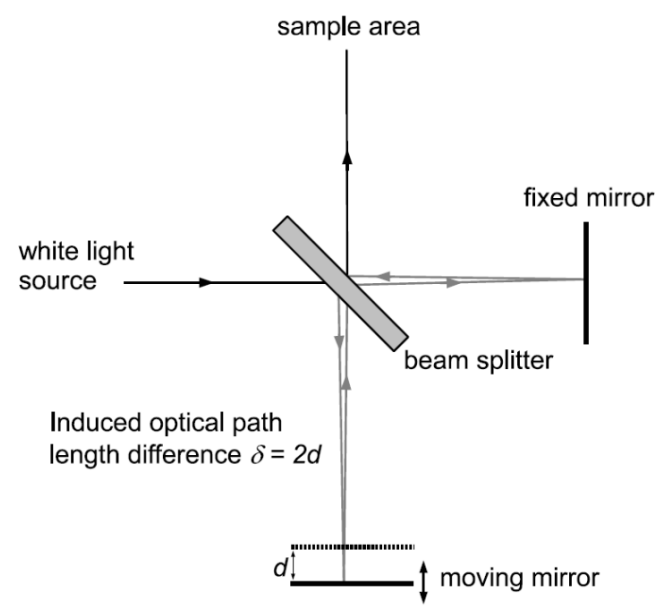

Figure 6.3 | Schematic illustration of a Fourier transform spectrometer, which is essentially a Michelson interferometer with one mirror movable.

Another technique often used to get a sensitive EQE is the Fourier-transform Photocurrent Spectroscopy (FTPS). Instead of using a monochromatic light, this technique uses a dispersive white light to illuminate the sample. The white light is timemodulated at each wavelength via a Fourier-transform spectrometer (see Figure 6.3). In the interferometer, the incident light is split into two by a beamsplitter, and subsequently reflected by two mirrors. Depending on the distance of two mirror, a path length difference $\delta=2 \mathrm{~d}$ is created between the two reflection beams. Accordingly, constructive or destructive interference occurs when them come together. The analytical expression for the intensity of the output light at a specified wavenumber $\sigma$ is

$$
s(\sigma)(1+\cos (2 \pi \sigma \delta))
$$

where $s(\sigma)$ is the incident light irradiance of wavenumber $\sigma$. If this output beam in 
incident on a detector with a response $R(\sigma)$, the detector produces a photocurrent $I(\delta)$ as

$$
I(\delta)=\int_{0}^{\infty} s(\sigma)(1+\cos (2 \pi \sigma \delta)) d \sigma
$$

The response at each wavelength $R(\sigma) s(\sigma)$ can be obtained by convolving the output signal.

$$
R(\sigma) s(\sigma)=4 \int_{0}^{\infty}\left(I(\delta)-\frac{1}{2} I(0)\right) \cos (2 \pi \sigma \delta) d \delta
$$

The actual operation is to input the photocurrent to the Fourier-transform instrument after passing through the preamplifier. And the instrument can do the inverse Fourier transform easily.

For a monochromator, the light output is positively correlated to the slit width, resulting in a trade-off between the spectral resolution and the signal-to-noise ratio. In contrast, FTPS allows very fine spectral resolution while maintaining a high signalto-noise ratio. However, since the finite mirror distance and the finite scanning time, the spectral bandwidth of FTPS may be limited. Moreover, if a sample has uneven photocurrent responses over frequencies, the photocurrent spectrum would exhibit deformation, because every $\sigma$ is correlated to a different $f$ while $f$ is typically around 5 $\mathrm{kHz}$ or higher. A correction procedure is usually performed at several mirror scanning speeeds $\mathrm{V}_{1}, \mathrm{~V}_{2}, \ldots, \mathrm{V}_{n}$. For each wavenumber, this introduces frequencies $f_{1}=2 \sigma V_{1}$, $f_{2}=2 \sigma V_{2}, \ldots, f_{n}=2 \sigma V_{n}$, which is a part of the relative frequency response. Here, 'relative' means no absolute value but ratios between each frequencies. By changing the wavenumber, we can get a continuous response by matching the frequency response curves in their overlapping frequency region.

\subsection{Steady-state spectrum}

\subsubsection{Spectrograph}

A spectrograph (spectrometer) is an instrument used to measure the light intensity over a specific wavelength. As mentioned in the last section, the method to measure a wavelength-dependent parameter can be either monochromatic or Fouriertransform. In this section, we focus on only the spectrograph using a monochromatic technique. As shown in Figure 6.2, a diffraction grating or a prism is used to disperse the light according to the wavelength. The dispersive light with different colors is focused into a detector, which is usually a CCD array. The response of the detector at each wavelength can be recorded and shown in a computer as a spectrum. The spectrograph (Shamrock 303i) in our lab is equipped with a silicon array detector (Andor Newton EM-CCD) and an InGaAs detector (Andor). The silicon detector works in the wavelength range of $300 \mathrm{~nm}-1100 \mathrm{~nm}$ and is cooled to $-45^{\circ} \mathrm{C}$ during the measurement. the InGaAs detector works in $800 \mathrm{~nm}-1700 \mathrm{~nm}$ and is cooled to $-80^{\circ} \mathrm{C}$ during the measurement. A Mercury lamp is used for wavelength position calibration and a standard halogen lamp (AvaLight-HAL-S-Mini) is used for wavelength intensity calibration. 


\subsubsection{UV-VIS-NIR absorption}

Measuring the material absorption is a standard technique that allows easy yet effective characterisation of material features. In our lab, we used a commercial setup (Perkin Elmer Lambda 950 UV/VIS/NIR). In this setup, the light of a Tungsten lamp or a Deuterium lamp passes a monochromator and comes out as monochromatic light. The monochromatic light is collimated and directed onto the sample. For transmission measurement, the sample is put before an integrating sphere and the transmitted light enters the integrating sphere. For reflection measurement, the sample is put after the integrating sphere and the reflected light enters the intergrating sphere. There are two detectors under the integrating sphere. A photomultiplier tube (PMT) is used in the UV/VIS range while a lead sulfide $(\mathrm{PbS})$ detector is used in the NIR range.

\subsubsection{Photoluminescence and quantum yield measurement}

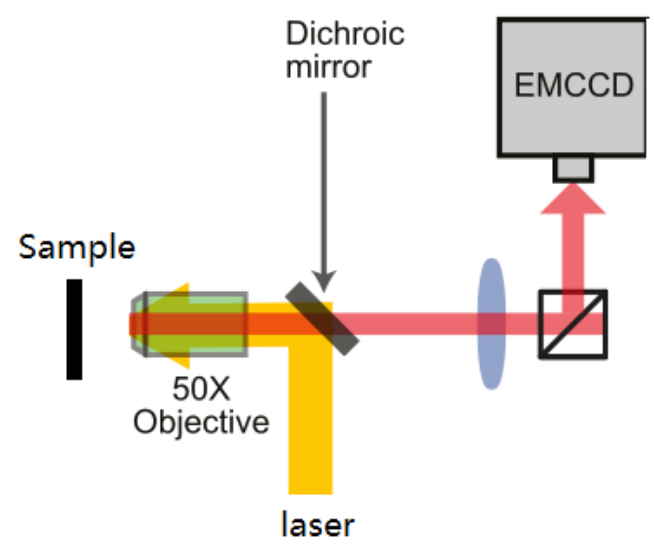

Figure 6.4 | A schematic for a PL measurement.

The PL is excited by a collimated laser-diode-pumped laser module. A short-pass filter is used after the laser to reduce the tail light. A long-pass filter is used before the spectrograph to protect the detector. The PL excitation and detection were performed via an objective, a $45^{\circ}$ placed long-pass dichroism glass and optical fibers. For biasdependent PL measurement, a source meter Keithley 2400 was connected to the photovoltaic devices to sweep the voltage bias and to record the current.

When measuring the PLQY of solids, a "Direct \& Indirect Excitation" method is used to reduce the scattering impact. The measured spectrum is composed of that from the un-absorbed pump laser and that from the sample emission. We refer to the amount of un-absorbed pump laser as L, and that of the emitted light as P. In the "off" experiment, we assume that a fraction $\mu$ of the scattered light is absorbed by the sample. In the "on" experiment, we assume that a fraction A of the incident light is absorbed by 

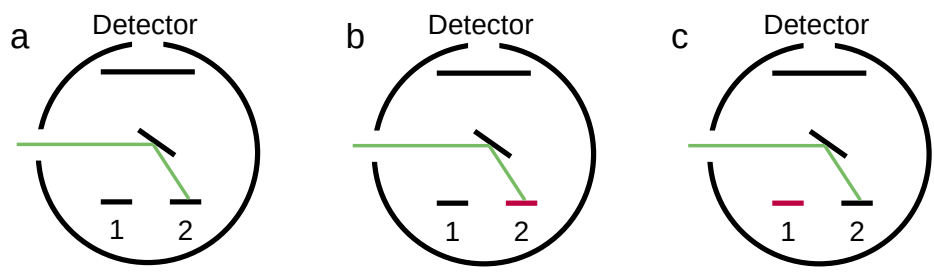

Figure 6.5 | Schematic for a PLQY measurement of film samples. (a) Blank: reference samples in both sample position 1 and 2; (b) On: the test sample in position 2 and the reference sample in position 1; (c) Off: the reference sample in position 2 and the test sample in position 1

the sample. We have

$$
\begin{array}{r}
L_{\text {off }}=L_{\text {Blank }}(1-\mu) \\
L_{\text {on }}=L_{\text {Blank }}(1-A)(1-\mu)
\end{array}
$$

From these two equations, we have

$$
A=1-\frac{L_{o n}}{L_{o f f}}
$$

In the "off" experiment, the number of photons striking the detector is $\mathrm{L}_{o f f}+\mathrm{P}_{o f f}$. In the "on" experiment, this part of light will be decreased to (1-A) $\times\left(\mathrm{L}_{\text {off }}+\mathrm{P}_{\text {off }}\right)$. However, in the "on" experiment, we also have light emitted directly due to the absorption of collimated laser light, which is $\mathrm{PLQY} \times \mathrm{L}_{\text {Blank }} \times \mathrm{A}$. Hence

$$
L_{o n}+P_{o n}=(1-A) \times\left(L_{o f f}+P_{o f f}\right)+P L Q Y \times L_{\text {Blank }} \times A
$$

From this equation, the PLQY can be calculated as

$$
P L Q Y=\frac{P_{o n}-(1-A) P_{o f f}}{L_{\text {Blank }} A}=\frac{P_{o n}-P_{o f f} \frac{L_{o n}}{L_{o f f}}}{L_{\text {Blank }}\left(1-\frac{L_{o n}}{L_{\text {off }}}\right)}
$$

\subsubsection{External quantum efficiency for electroluminescence}

As shown in Figure 6.6, the electroluminescence (EL) of a sample is foucused into a spectrograph for EL spectra. The setup is the same as bias-dependent PL measurement, as shown in the last subsection. A large area silicon photodiode (Hamamatsu $1010 \mathrm{~B}$ ) is used for the measurement of $\mathrm{EQE}_{\mathrm{EL}}$ (Figure 6.6). A Keithley 2400 SourceMeter is used to apply voltage and record the injected current. A Keithley 485 Picoammeter is used to measure the signal of the silicon photodiode for the emitted light intensity. The $\mathrm{EQE}_{E L}$ is calculated according to a wavelength response which depends on both the $\mathrm{EQE}_{P V}$ of the silicon photodiode and the instrument loss. The response is calibrated with a standard LED. 


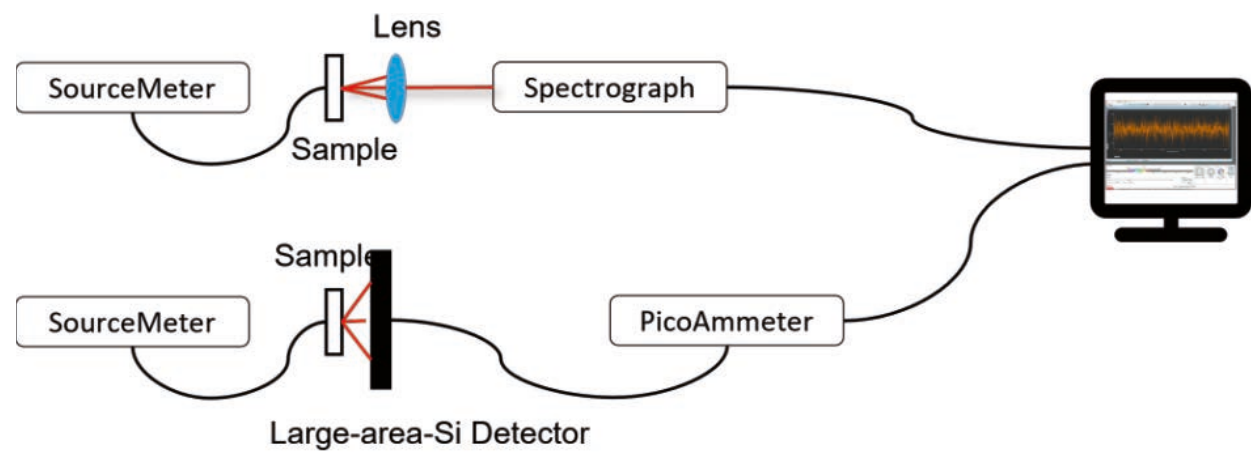

Figure 6.6 | Schematic for the $\mathrm{EQE}_{E L}$ measurement.

\subsection{Time-resolved measurements}

\subsubsection{Transient absorption spectroscopy}

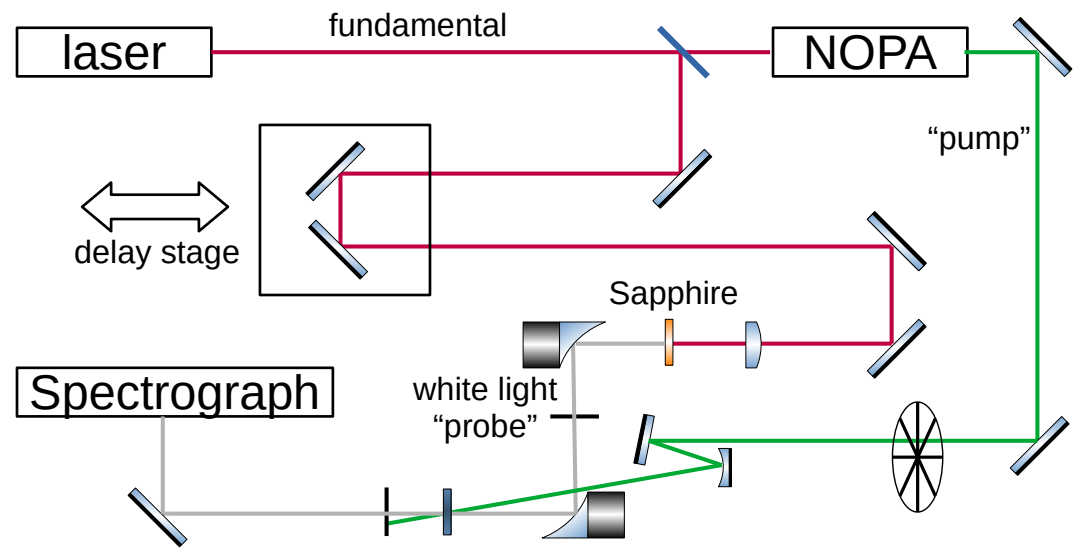

Figure 6.7 | Schematic illustration of the transient absorption measurement.

Each excited state has its distinct absorption and emission. However, some excited states have very short lifetime and constitute very little proportion in the steady state. To map the evolution of those states over time, an excited-state absorption measurement called transient absorption spectroscopy (TAS) is often used by researchers. In TAS, the absorbance over a specific wavelength range of a sample is measured as a function of time after excitation by a flash of light. A typical technique for measurement is called pump-probe spectroscopy (PP), in which both the light for excitation ('pump') and the light for measuring the absorbance ('probe') are generated by a pulsed laser. The 'pump' is typically a broad-band pulse generated by a noncollinear optical parametric amplifier (NOPA) and a fundamental laser. If no short pulse duration is needed, an optical parametric amplifier (OPA) can be used to gen- 


\section{Ch. 6 | Methods}

erate narrowband pump pulses. The 'probe' is typically a supercontinuum (whitelight pulse) generated by tightly focusing intense ultrashort pulses in crystals such as yttrium aluminum garnet (YAG), lithium fluoride (LiF), or sapphire. The time delay between the pump and probe light can be mechanical (typically between ps and ns) or electronic (typically between ns and ms). PP spectra are obtained by comparing the transmission of the probe light (white light supercontinuum) when the sample is excited, $\mathrm{T}_{\text {pumpon }}$, with the transmission when the pump is blocked by the chopper, $\mathrm{T}_{\text {pumpoff }}$

$$
\frac{\Delta T}{T}=\frac{T_{\text {pump on }}-T_{\text {pump off }}}{T_{\text {pump off }}}
$$

As illustrated in Figure 6.8, the main features in typical PP spectra include the groundstate bleaching (GSB), the stimulated emission (SE), and the photo-induced absorption (PIA). These features come from the disappearance or the appearance of electronics states. By decomposing the raw signal into these individual components, we can observe the evolation of the corresponding states. A typical analysis may involve mathematical models of physical processes with the help of other steady or timeresolved spectroscopy.

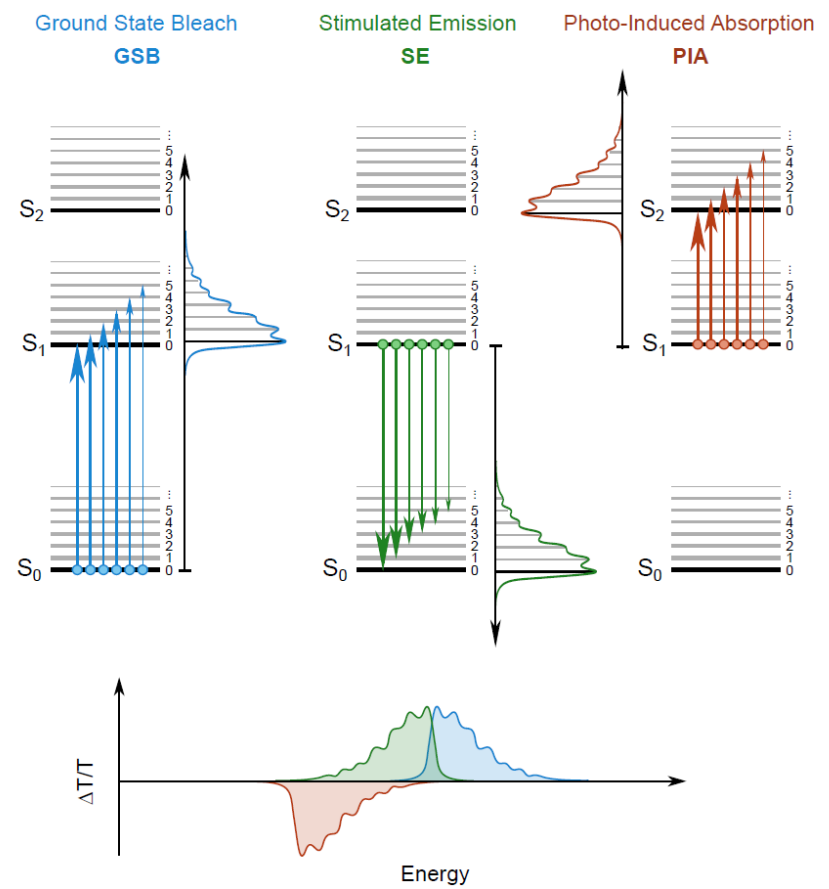

Figure 6.8 | Schematic illustration of features contributing to a transient absorption spectrum. The ground state bleaching (blue) comes from $\mathrm{S}_{0} \rightarrow \mathrm{S}_{n}$ transition. The stimulated emission (green) originates from the inverse process $S_{0} \leftarrow S_{1}$. The photoinduced absorption (red) is from $\mathrm{S}_{1} \rightarrow \mathrm{S}_{n}$ transitions. Credit: Dr. Andreas Jakowetz.

The GSB signal is the result of reduced absorption when the sample is excited. The ab- 
sorption of molecules in the ground state can be measured by steady-state absorption spectroscopy, as described in subsection 6.2.2. After excitation, the depopulation of the ground state causes the transmitted probe light less absorbed at the energies that correspond to the ground state absorption hence a GSB. $\Delta$ T gives a positive signal with a shape similar to the steady-state absorption spectrum. GSB cannot differentiate the type of excited states. Its intensity is proportional to the density of total excited states.

The SE signal is the result of stimulated emission from molecules in an excited state. The excited state can randomly decay to the ground state by emitting a photon, which is usually measured in the steady-state PL or the time-correlated single-photon counting (TCSPC). However, the radiative decay can be stimulated by a photon of energy corresponding to the transition to a lower energy state and emits a second photon of the same energy. This process is called stimulated emission. The SE caused by the probe light is detected as increased transmission, thus producing a positive $\Delta \mathrm{T}$. Its shape roughly matches the PL spectrum and its intensity represents the population of excited states that can undergo radiative transitions.

The PIA signal is the result of absorption by excited states that transition to higher excited states. The excited states provide new transitions and absorb additional probe light, which results in a negative $\Delta \mathrm{T}$. In contrast to the SE signal, PIA also comes from states that can not undergo radiative transitions to the ground state, such as triplet excitons and charges.

\subsubsection{Time-delayed collection field}

Time-delayed collection field (TDCF) is a transient technique to monitor the evolution of charge carriers in a device after excitation by a flah of light. The TDCF measurement has similarities to the transient photocurrent (TPC) and the transient photovoltage (TPV) measurements, but is more powerful in investigating the charge generation and recombination process. Compared with TAS, TDCF typically provides a longer observation time scale for the long-lived charge carriers in ready-made devices.

In TDCF, the sample device is excited by a plused laser. Before the excitaion, a prebias is applied and maintained on the device. The charge generation process accomplishes during the pre-bias. After a specified time delay, a large reverse collection field is applied on the devcie to extract all remaining charges before significant recombination. Monitoring the extraction current can provide information about charge density in the device. By varying the pre-bias and making the time delay short, we can investigate the field dependence of charge generation. By varying the time delay and keeping the pre-bias, we can study the recombination process of free charge carriers. As indicated in Figure 6.9, the pre-bias and collection field are applied by a low-jitter function generator. A synchronization and delay generator (SDG) is used to trigger both the laser pulse and the function generator. Fast charge extraction requires a small measurement resistor (10 Ohms) and a small capacitance ( $100 \mathrm{pF})$ which results in a short and large recharging current of ca. 1 A within 2.7 ns [146]. A high-definition oscilloscope with very low noise input amplifiers is needed to reach an overall high sensitivity for monitoring the extraction current. 
Ch. 6 | Methods
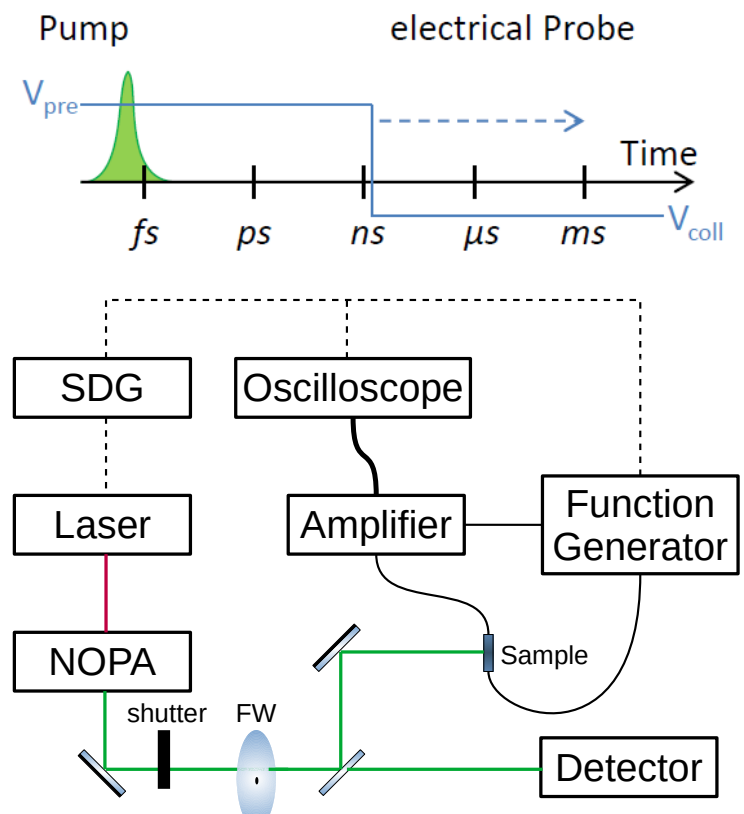

Figure 6.9 | Schematic illustration of the TDCF measurement. The synchronization and delay generator (SDG) controls the laser and delivers a trigger to the function generator and the oscilloscope. The filter wheel (FW) controls the excitation intensity. Credit: Dr. Jona Kurpiers. 


\section{CHAPTER 7}

Results and included pappers

The results obtained from my research leading up to this thesis are presented in the form of three scientific papers published in peer-reviewed journals and a manuscript. 
Ch. 7 | Results and included pappers

\subsection{List of publications}

Papers appended in the thesis:

[I] Barrierless Free Charge Generation in the High-Performance PM6:Y6 Bulk Heterojunction Non-Fullerene Solar Cell

Lorena Perdigón-Toro, Huotian Zhang, Anastasia Markina, Jun Yuan, Seyed Mehrdad Hosseini, Christian M Wolff, Guangzheng Zuo, Martin Stolterfoht, Yingping Zou, Feng Gao, Denis Andrienko, Safa Shoaee, Dieter Neher

Advanced Materials, 32, 1906763 (2020).

Contribution: I fabricated the devices, did the initial characterization, and performed the temperature-dependent measurements. I did the TDCF measurements and analyzed the data with L.P.-T. I contributed to the revision of the manuscript.

[II] From Generation to Extraction: A Time-Resolved Investigation of Photophysical Processes in Non-fullerene Organic Solar Cells

Rokas Jasiūnas, Huotian Zhang, Jun Yuan, Xuehong Zhou, Deping Qian, Yingping Zou, Andrius Devizis, Juozas Sulskus, Feng Gao, Vidmantas Gulbinas The Journal of Physical Chemistry C, 124, 21283-21292 (2020).

Contribution: I participated in conceiving the idea. I fabricated the devices. I contributed to the revision of the manuscript.

[III] Effect of the Energy Offset on the Charge Dynamics in Non-fullerene Organic Solar Cells

Yunhao Cai, Huotian Zhang, Linglong Ye, Rui Zhang, Jinqiu Xu, Kangning Zhang, Pengqing Bi, Tengfei Li, Kangkang Weng, Ke Xu, Jianlong Xia, Qinye Bao, Feng Liu, Xiaotao Hao, Songting Tan, Feng Gao, Xiaowei Zhan, Yanming Sun ACS Applied Materials \& Interfaces, 12, 43984-43991 (2020).

Contribution: I did the EL, PL and FTPS-EQE measurements, and analyzed the voltage loss data. I contributed to the first draft and the revision of the manuscript.

[IV] The relation between Fill Factor and Open-circuit Voltage Loss in Non-fullerene Organic solar cells

Huotian Zhang, Rui Zhang, Xuehong Zhou, Jun Yuan, Rokas Jasiūnas, Wenchao Huang, Vidmantas Gulbinas, Arkady Yartsev, Feng Gao

Manuscript

Contribution: I initiated and coordinate the project. I collected and analyzed the data. I wrote the first draft of the manuscript. 


\section{Additional publications not included in the thesis:}

- Optical gaps of organic solar cells as a reference for comparing voltage losses Yuming Wang, Deping Qian, Yong Cui, Huotian Zhang, Jianhui Hou, Koen Vandewal, Thomas Kirchartz, Feng Gao Advanced Energy Materials, 8, 1801352 (2018).

Contribution: I contributed to the discussion and the revision of the manuscript.

- A near-infrared photoactive morphology modifier leads to significant current improvement and energy loss mitigation for ternary organic solar cells Lingling Zhan, Shuixing Li, Huotian Zhang, Feng Gao, Tsz-Ki Lau, Xinhui Lu, Danyang Sun, Peng Wang, Minmin Shi, Chang-Zhi Li, Hongzheng Chen Advanced Science, 5, 1800755 (2018)

Contribution: I measured the FTPS-EQE and the EQE of EL. I did the voltage loss calculation.

- Oriented quasi-2D perovskites for high performance optoelectronic devices Rong Yang, Renzhi Li, Yu Cao, Yingqiang Wei, Yanfeng Miao, Wen Liang Tan, Xuechen Jiao, Hong Chen, Liangdong Zhang, Qing Chen, Huotian Zhang, Wei Zou, Yuming Wang, Ming Yang, Chang Yi, Nana Wang, Feng Gao, Christopher R McNeill, Tianshi Qin, Jianpu Wang, Wei Huang

Advanced Materials, 30, 1804771 (2018)

Contribution: I measured the EQE of EL.

- Enabling low voltage losses and high photocurrent in fullerene-free organic photovoltaics

Jun Yuan, Tianyi Huang, Pei Cheng, Yingping Zou, Huotian Zhang, Jonathan Lee Yang, Sheng-Yung Chang, Zhenzhen Zhang, Wenchao Huang, Rui Wang, Dong Meng, Feng Gao, Yang Yang

Nature Communications, 10, 44204 (2019)

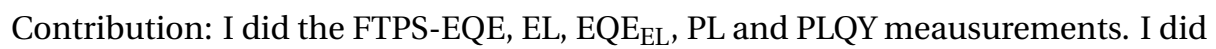
the voltage loss calculation and the time-resolved PL analysis. I wrote a part of the first draft and contributed to the revision of the final manuscript.

- The crucial role of end group planarity for fused-ring electron acceptors in organic solar cells

Jeromy J Rech, Nicole Bauer, David Dirkes, Joseph Kaplan, Zhengxing Peng, Huotian Zhang, Long Ye, Shubin Liu, Feng Gao, Harald Ade, Wei You

Materials Chemistry Frontiers, 3, 1642-1652 (2019)

Contribution: I did the PLQY measurement.

- Sulfur vs. tellurium: the heteroatom effects on the nonfullerene acceptors

Lei Yang, Linqing Qin, Yunxiao Xu, Huotian Zhang, Lei Lv, Kepeng Chen, Xinyu Sui, Yangguang Zhong, Yuan Guo, Feng Gao, Jianzhang Zhao, Yuhao Li, Xinfeng Liu, Yuanping Yi, Xinhui Lu, Aidong Peng, Hui Huang

Science China Chemistry, 62, 897-903 (2019) 
Ch. 7 | Results and included pappers

Contribution: I measured the FTPS-EQE and the EQE of EL. I did the voltage loss calculation and the reciprocity fitting.

- Realizing Efficient Charge/Energy Transfer and Charge Extraction in FullereneFree Organic Photovoltaics via a Versatile Third Component

Hao-Wen Cheng, Huotian Zhang, Yu-Che Lin, Nian-Zu She, Rui Wang, Chung-Hao Chen, Jun Yuan, Cheng-Si Tsao, Atsushi Yabushita, Yingping Zou, Feng Gao, Pei Cheng, Kung-Hwa Wei, Yang Yang

Nano Letters, 19, 5053-5061 (2019)

Contribution: I did the FTPS-EQE, EL, EQE $E_{E L}$, PL and PLQY meausurements. I did the voltage loss calculation.

- Experimentally Validated Hopping-Transport Model for Energetically Disordered Organic Semiconductors

Tanvi Upreti, Yuming Wang, Huotian Zhang, Dorothea Scheunemann, Feng Gao, Martijn Kemerink

Physical Review Applied, 12, 64039 (2019)

Contribution: I fabricated the devices. I did the temperature-dependent JV measurement.

- Bright free exciton electroluminescence from Mn-doped two-dimensional layered perovskites

Liangdong Zhang, Tao Jiang, Chang Yi, Jiquan Wu, Xiao-Ke Liu, Yarong He, Yanfeng Miao, Ya Zhang, Huotian Zhang, Xinrui Xie, Peng Wang, Renzhi Li, Feng Gao, Wei Huang, Jianpu Wang

The Journal of Physical Chemistry Letters, 10, 3171-3175 (2019)

Contribution: I did the PLQY measurement.

- Tuning the electron-deficient core of a non-fullerene acceptor to achieve over $17 \%$ efficiency in a single-junction organic solar cell

Can Zhu, Jun Yuan, Fangfang Cai, Lei Meng, Huotian Zhang, Honggang Chen, Jing Li, Beibei Qiu, Hongjian Peng, Shanshan Chen, Yunbin Hu, Changduk Yang, Feng Gao, Yingping Zou, Yongfang Li

Energy \& Environmental Science, 13, 2459-2466 (2020)

Contribution: I measured the FTPS-EQE and the EQE of EL. I did the voltage loss calculation and the reciprocity fitting.

- Promoting charge separation resulting in ternary organic solar cells efficiency over $17.5 \%$

Qing Ma, Zhenrong Jia, Lei Meng, Jinyuan Zhang, Huotian Zhang, Wenchao Huang, Jun Yuan, Feng Gao, Yan Wan, Zhanjun Zhang, Yongfang Li

Nano Energy, 78, 105272 (2020)

Contribution: I measured the FTPS-EQE and the EQE of EL. I did the voltage loss calculation and the reciprocity fitting.

- Reliability of charge carrier recombination data determined with charge extraction methods 
Juliane Kniepert, Andreas Paulke, Lorena Perdigón-Toro, Jona Kurpiers, Huotian Zhang, Feng Gao, Jun Yuan, Yingping Zou, Vincent M Le Corre, L Jan Anton Koster, Dieter Neher Journal of Applied Physics, 126, 205501 (2019)

Contribution: I fabricated and characterized the devices.

- Reducing voltage losses in the A-DA' DA acceptor-based organic solar cells Jun Yuan, Huotian Zhang, Rui Zhang, Yuming Wang, Jianhui Hou, Mario Leclerc, Xiaowei Zhan, Fei Huang, Feng Gao, Yingping Zou, Yongfang Li

Chem, , (2020)

Contribution: I contributed to the discussion, draft and revision of the manuscript.

- Understanding energetic disorder in electron-deficient-core-based non-fullerene solar cells

Jun Yuan, Chujun Zhang, Honggang Chen, Can Zhu, Sin Hang Cheung, Beibei Qiu, Fangfang Cai, Qingya Wei, Wei Liu, Hang Yin, Rui Zhang, Jidong Zhang, Ye Liu, Huotian Zhang, Weifang Liu, Hongjian Peng, Junliang Yang, Lei Meng, Feng Gao, Shukong So, Yongfang Li, Yingping Zou

Science China Chemistry, 63, 1159-1168 (2020)

Contribution: I contributed to the discussion and revision of the manuscript.

- Emerging approaches in enhancing the efficiency and stability in non-fullerene organic solar cells

Fuwen Zhao, Huotian Zhang, Rui Zhang, Jun Yuan, Dan He, Yingping Zou, Feng Gao

Advanced Energy Materials, 10, 2002746 (2020)

Contribution: I contributed to the discussion, draft and revision of the manuscript.

- Highly efficient fused ring electron acceptors based on a new undecacyclic core Fuwen Zhao, Dan He, Jingming Xin, Huotian Zhang, Jixiang Zhou, Baojun Lin, Yongju He, Jiang Li, Wei Ma, Bao Li, Feng Gao, Yongfang Li, Chunru Wang Materials Chemistry Frontiers, 5, 2001-2006 (2021)

Contribution: I measured the FTPS-EQE and the EQE of EL. I did the voltage loss calculation.

- Revealing Morphology Evolution in Highly Efficient Bulk Heterojunction and Pseudo-Planar Heterojunction Solar Cells by Additives Treatment

Qiannan He, Wangping Sheng, Ming Zhang, Guodong Xu, Peipei Zhu, Huotian Zhang, Zhaoyang Yao, Feng Gao, Feng Liu, Xunfan Liao, Yiwang Chen

Advanced Energy Materials, 11, 2003390 (2021)

Contribution: I did the voltage loss calculation and analysis.

- Non-fullerene acceptors with branched side chains and improved molecular packing to exceed $18 \%$ efficiency in organic solar cells

Chao Li, Jiadong Zhou, Jiali Song, Jinqiu Xu, Huotian Zhang, Xuning Zhang, Jing Guo, Lei Zhu, Donghui Wei, Guangchao Han, Jie Min, Yuan Zhang, Zengqi Xie, 
Ch. 7 | Results and included pappers

Yuanping Yi, He Yan, Feng Gao, Feng Liu, Yanming Sun

Nature Energy, 6, 605-613 (2021)

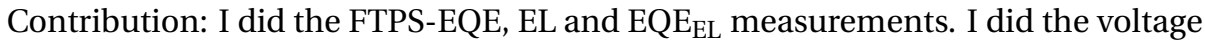
loss calculation. I contributed to the discussion and revision of the manuscript.

- Carrier Mobility Dynamics under Actual Working Conditions of Organic Solar Cells

Rokas Jasiūnas, Vidmantas Jasinskas, Huotian Zhang, Tanvi Upreti, Feng Gao, Martijn Kemerink, Vidmantas Gulbinas

The Journal of Physical Chemistry C, 125, 14567-14575 (2021)

Contribution: I fabricated the devices. 


\subsection{Summary of included papers}

\section{Paper I}

\section{Barrierless Free Charge Generation in the High-Performance PM6:Y6 Bulk Het- erojunction Non-Fullerene Solar Cell}

\section{Summary}

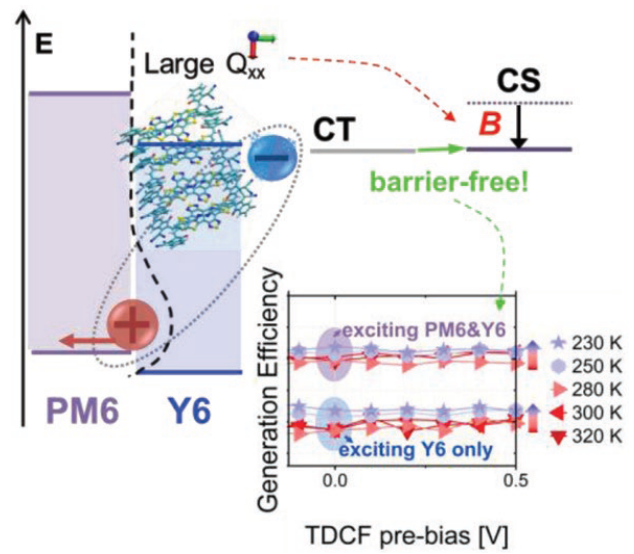

The efficiency of photocurrent generation is studied in the high-efficiency non-fullerene PM6:Y6 blend, using a combination of field- and temperature-dependent optoelectronic measurements. These experiments reveal barrierless free charge generation, despite a small driving force. Theoretical modeling suggests the existence of a large electrostatic interfacial field, which repels charges from the donor-acceptor interface.

\section{Author's contribution}

I fabricated the devices, did the initial characterization, and performed the temperaturedependent measurements. I did the TDCF measurements and analyzed the data with L.P.-T. I contributed to the revision of the manuscript. 
Ch. 7 | Results and included pappers

\section{Paper II}

From Generation to Extraction: A Time-Resolved Investigation of Photophysical Processes in Non-fullerene Organic Solar Cells

\section{Summary}

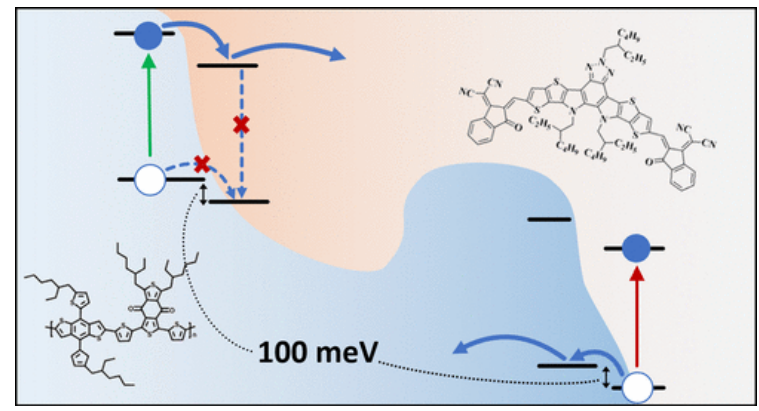

The photophysical process from sub-ps to $\mu$ s is studied in organic solar cells based on three non-fullerene combinations with negligible HOMO offsets, using several transient investigation techniques. The experiments show inefficient hole transfer and enhanced geminate recombination for the low offset system.

\section{Author's contribution}

I participated in conceiving the idea. I fabricated the devices. I contributed to the revision of the manuscript. 


\section{Paper III}

Effect of the Energy Offset on the Charge Dynamics in Non-fullerene Organic Solar Cells

\section{Summary}
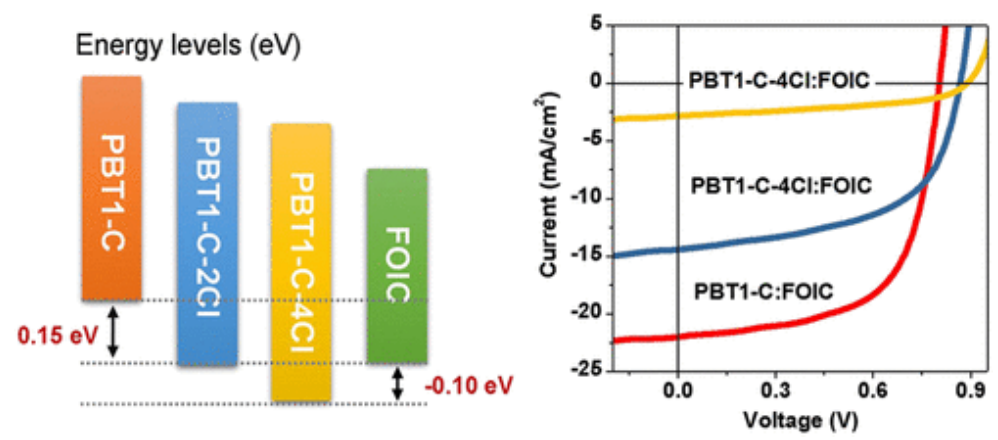

Three devices are presented with the HOMO offset changed from $0.15 \mathrm{eV}$ to $-0.1 \mathrm{eV}$ by polymer chlorination, which is cross verified by CV and UPS. This work indicates the feasibility of the free carrier generation and the following charge separation under the condition of a negative offset.

\section{Author's contribution}

I did the EL, PL and FTPS-EQE measurements, and analyzed the voltage loss data. I contributed to the first draft and the revision of the manuscript. 
Ch. 7 | Results and included pappers

\section{Paper IV}

The relation between Fill Factor and Open-circuit Voltage Loss in Non-fullerene Organic solar cells

\section{Summary}

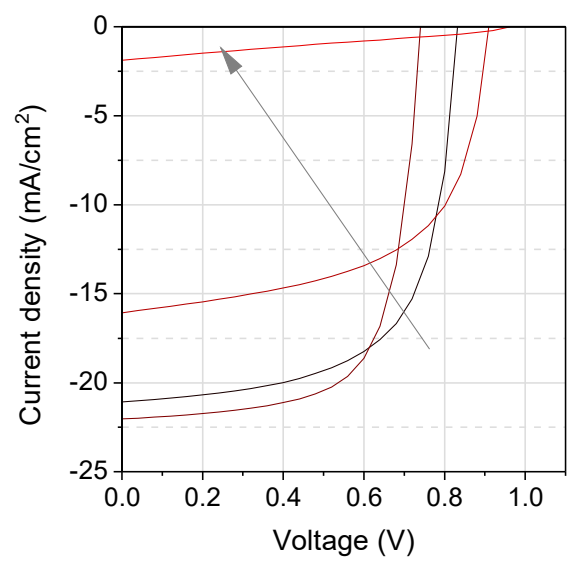

A large number of data about the FF and the $\mathrm{V}_{\mathrm{OC}}$ are summarized. The data together with theoritical simulations show no competition between FF and $\mathrm{V}_{\mathrm{OC}}$ in a non-geminate recombination dominated condition. However, field-dependent charge dissociation may harm FF when geminate recombination is dominating.

\section{Author's contribution}

I initiated and coordinate the project. I collected and analyzed the data. I wrote the first draft of the manuscript. 
1. For Space Studies (GISS), N. G. I. Global Temperature https : //climate . nasa . gov/vital-signs/global-temperature/ (2021) (cit. on pp. 1, 2).

2. Smith, J. B. et al. Assessing dangerous climate change through an update of the Intergovernmental Panel on Climate Change (IPCC) "reasons for concern". Proceedings of the national Academy of Sciences 106, 4133-4137 (2009) (cit. on p. 1).

3. Frölicher, T. L., Fischer, E. M. \& Gruber, N. Marine heatwaves under global warming. Nature 560, 360-364 (2018) (cit. on p. 1).

4. Tapley, B. D. et al. Contributions of GRACE to understanding climate change. Nature climate change 9, 358-369 (2019) (cit. on p. 1).

5. Karl, T. R. \& Trenberth, K. E. Modern global climate change. Science 302, 17191723. eprint: https ://science . sciencemag. org/content/302/5651/1719. full . pdf. https://science.sciencemag.org/content/302/5651/1719 (2003) (cit. on p. 1).

6. Letcher, T. M. in Managing Global Warming 3-15 (Elsevier, 2019) (cit. on p. 1).

7. Wilcke, R. A. I. et al. The extremely warm summer of 2018 in Sweden-set in a historical context. Earth System Dynamics 11, 1107-1121 (2020) (cit. on p. 1).

8. Pascale, S., Kapnick, S. B., Delworth, T. L. \& Cooke, W. F. Increasing risk of another Cape Town "Day Zero" drought in the 21st century. Proceedings of the National Academy of Sciences 117, 29495-29503 (2020) (cit. on p. 1).

9. Philip, S. et al. Attributing the 2017 Bangladesh floods from meteorological and hydrological perspectives. Hydrology and Earth System Sciences 23, 1409-1429 (2019) (cit. on p. 1).

10. Keellings, D. \& Ayala, J. J. H. Extreme rainfall associated with Hurricane Maria over Puerto Rico and its connections to climate variability and change. Geophysical Research Letters 46, 2964-2973 (2019) (cit. on p. 1).

11. Doss-Gollin, J., Farnham, D. J., Lall, U. \& Modi, V. How unprecedented was the February 2021 Texas cold snap? Environmental Research Letters 16, 064056 (2021) (cit. on p. 1).

12. Xu, Y., Ramanathan, V. \& Victor, D. G. Global warming will happen faster than we think 2018 (cit. on p. 1). 
13. p.l.c., B. Statistical Review of World Energy https : / / www . bp . com / en / global / corporate / energy - economics / statistical-review - of - world-energy . html (2021) (cit. on p. 2).

14. (EIA), T. U. E. I. A. Greenhouse gases and the climate https : / /www . eia . gov/ energyexplained/energy-and-the-environment/greenhouse-gases-and-the-climate . php (2021) (cit. on p. 2).

15. Köhler, A. \& Bässler, H. Electronic processes in organic semiconductors: An introduction (John Wiley \& Sons, 2015) (cit. on pp. 4, 15, 34, 38, 40).

16. Inganäs, O. Organic photovoltaics over three decades. Advanced Materials 30, 1800388 (2018) (cit. on p. 4).

17. Friend, R. Organic materials for large area electronics in Materials Science Forum 608 (2009), 159-179 (cit. on p. 4).

18. Kallmann, H. \& Pope, M. Photovoltaic effect in organic crystals. The Journal of Chemical Physics 30, 585-586 (1959) (cit. on p. 5).

19. Zhang, T. et al. A thiadiazole-based conjugated polymer with ultradeep HOMO level and strong electroluminescence enables $18.6 \%$ efficiency in organic solar cell. Advanced Energy Materials, 2101705 (2021) (cit. on pp. 5, 46).

20. Li, C. et al. Non-fullerene acceptors with branched side chains and improved molecular packing to exceed $18 \%$ efficiency in organic solar cells. Nature Energy 6, 605-613 (2021) (cit. on pp. 5, 30, 34, 46).

21. Yuan, J. et al. Reducing voltage losses in the A-DA' DA acceptor-based organic solar cells. Chem (2020) (cit. on p. 5).

22. Richter, A. et al. Design rules for high-efficiency both-sides-contacted silicon solar cells with balanced charge carrier transport and recombination losses. Nature Energy 6, 429-438 (2021) (cit. on p. 5).

23. Green, M. et al. Solar Cell Efficiency Tables (Version 57). Progress in Photovoltaics: Research and Applications 29, 3-15 (2021) (cit. on p. 5).

24. Polverini, D., Field, M., Dunlop, E. \& Zaaiman, W. Polycrystalline silicon PV modules performance and degradation over 20 years. Progress in Photovoltaics: Research and Applications 21, 1004-1015 (2013) (cit. on p. 5).

25. Kirchartz, T. \& Rau, U. What makes a good solar cell? Advanced Energy Materials 8, 1703385 (2018) (cit. on pp. 6, 25).

26. Neamen, D. A. Semiconductor physics and devices (1992) (cit. on pp. 7, 10).

27. Würfel, P. \& Würfel, U. Physics of solar cells: from basic principles to advanced concepts (John Wiley \& Sons, 2016) (cit. on p. 9).

28. Nelson, J. A. The physics of solar cells (World Scientific Publishing Company, 2003) (cit. on p. 10).

29. Laboratory, T. N. R. E. Reference Air Mass 1.5 Spectra https : / / www . nrel . gov/ grid/solar-resource/spectra-am1.5.html (2021) (cit. on p. 12).

30. For Testing, A. S., on Weathering, M. C. G. \& Durability. Standard tables for reference solar spectral irradiances: direct normal and hemispherical on $37^{\circ}$ tilted surface (ASTM international, 2003) (cit. on p. 12).

31. Leary, G., Switzer, G., Kuntz, G. \& Kaiser, T. Comparison of xenon lamp-based and led-based solar simulators in 2016 IEEE 43rd Photovoltaic Specialists Conference (PVSC) (2016), 3062-3067 (cit. on p. 12).

32. Tress, W. in Organic Solar Cells 67-214 (Springer, 2014) (cit. on p. 15). 
33. Kuhn, H. A quantum-mechanical theory of light absorption of organic dyes and similar compounds. The Journal of chemical physics 17, 1198-1212 (1949) (cit. on p. 15).

34. Somoza, M. M. Wikimedia commons: Depiction of franck condon principle in absorption and fluorescence https ://commons . wikimedia.org/wiki/File: FranckCondon-diagram.png (cit. on p. 16).

35. Somoza, M. M. Wikimedia commons:Depiction of absorption and fluorescence progression due to changes in vibrational levels during electronic transition https://commons . wikimedia.org/wiki/File:Vibration-fluor-abs.png (cit. on p. 16).

36. Schwoerer, M. \&Wolf, H. C. Organic molecular solids (John Wiley \& Sons, 2007) (cit. on p. 15).

37. Felekidis, N. Effects of energetic disorder on the optoelectronic properties of organic solar cells (Linköping University Electronic Press, 2018) (cit. on p. 16).

38. McConnell, H. M. Intramolecular charge transfer in aromatic free radicals. The Journal of Chemical Physics 35, 508-515 (1961) (cit. on p. 17).

39. Grabowski, Z. R., Rotkiewicz, K. \& Rettig, W. Structural changes accompanying intramolecular electron transfer: focus on twisted intramolecular chargetransfer states and structures. Chemical Reviews 103, 3899-4032 (2003) (cit. on p. 17).

40. Ward, A. J. et al. The impact of driving force on electron transfer rates in photovoltaic donor-acceptor blends. Advanced Materials 27, 2496-2500 (2015) (cit. on pp. 17, 18, 35).

41. Lüssem, B., Riede, M. \& Leo, K. Doping of organic semiconductors. physica status solidi (a) 210,9-43. eprint: https://onlinelibrary .wiley.com/doi/pdf/10. 1002/pssa. 201228310. https : // onlinelibrary . wiley . com/doi/abs/10 . 1002/pssa. 201228310 (2013) (cit. on p. 17).

42. Mulliken, R. S. Molecular compounds and their spectra. III. The interaction of electron donors and acceptors. The Journal of Physical Chemistry 56, 801-822 (1952) (cit. on p. 17).

43. Brédas, J.-L., Norton, J. E., Cornil, J. \& Coropceanu, V. Molecular understanding of organic solar cells: the challenges. Accounts of Chemical Research 42, 16911699 (2009) (cit. on pp. 17, 36).

44. Vandewal, K. Interfacial charge transfer states in condensed phase systems. Annual Review of Physical Chemistry 67, 113-133 (2016) (cit. on p. 17).

45. Deibel, C., Strobel, T. \& Dyakonov, V. Role of the charge transfer state in organic donor-acceptor solar cells. Advanced materials 22, 4097-4111 (2010) (cit. on p. 17).

46. Vandewal, K., Tvingstedt, K., Gadisa, A., Inganäs, O. \& Manca, J. V. On the origin of the open-circuit voltage of polymer-fullerene solar cells. Nature materials $\mathbf{8}$, 904-909 (2009) (cit. on pp. 17, 29).

47. Goris, L. et al. Absorption phenomena in organic thin films for solar cell applications investigated by photothermal deflection spectroscopy. Journal of Materials Science 40, 1413-1418 (2005) (cit. on p. 17).

48. Vandewal, K. et al. The relation between open-circuit voltage and the onset of photocurrent generation by charge-transfer absorption in polymer: fullerene bulk heterojunction solar cells. Advanced Functional Materials 18, 2064-2070 (2008) (cit. on p. 17). 
49. Qian, D. et al. Design rules for minimizing voltage losses in high-efficiency organic solar cells. Nature materials 17, 703-709 (2018) (cit. on pp. 17, 18, 25, 29, 31).

50. Loi, M. A. et al. Charge transfer excitons in bulk heterojunctions of a polyfluorene copolymer and a fullerene derivative. Advanced Functional Materials 17, 2111-2116 (2007) (cit. on p. 18).

51. Tvingstedt, K. et al. Electroluminescence from charge transfer states in polymer solar cells. Journal of the American Chemical Society 131, 11819-11824 (2009) (cit. on p. 18).

52. Song, Y., Clafton, S. N., Pensack, R. D., Kee, T. W. \& Scholes, G. D. Vibrational coherence probes the mechanism of ultrafast electron transfer in polymerfullerene blends. Nature communications 5, 1-7 (2014) (cit. on pp. 18, 35).

53. Provencher, F. et al. Direct observation of ultrafast long-range charge separation at polymer-fullerene heterojunctions. Nature communications $\mathbf{5}, 1-11$ (2014) (cit. on pp. 18, 35).

54. Zhong, Y. et al. Sub-picosecond charge-transfer at near-zero driving force in polymer:non-fullerene acceptor blends and bilayers. Nature Communications 11, 833. http: //www . nature . com/articles/s41467-020-14549-w (Dec. 2020) (cit. on pp. 18, 35).

55. Marcus, R. A. Electron transfer reactions in chemistry. Theory and experiment. Reviews of Modern Physics 65, 599 (1993) (cit. on p. 18).

56. Coffey, D. C. et al. An optimal driving force for converting excitons into free carriers in excitonic solar cells. The Journal of Physical Chemistry C 116, 89168923 (2012) (cit. on pp. 18, 35).

57. Jakowetz, A. C. et al. What controls the rate of ultrafast charge transfer and charge separation efficiency in organic photovoltaic blends. Journal of the American Chemical Society 138, 11672-11679 (2016) (cit. on p. 18).

58. Weller, A. Photoinduced electron transfer in solution: exciplex and radical ion pair formation free enthalpies and their solvent dependence. Zeitschrift für Physikalische Chemie 133, 93-98 (1982) (cit. on p. 18).

59. Liu, J. et al. Fast charge separation in a non-fullerene organic solar cell with a small driving force. Nature Energy 1, 1-7 (2016) (cit. on p. 18).

60. Nakano, K. et al. Anatomy of the energetic driving force for charge generation in organic solar cells. Nature communications 10, 1-10 (2019) (cit. on p. 18).

61. Classen, A. et al. The role of exciton lifetime for charge generation in organic solar cells at negligible energy-level offsets. Nature Energy 5, 711-719 (2020) (cit. on pp. 18, 29, 30, 34).

62. Karuthedath, S. et al. Intrinsic efficiency limits in low-bandgap non-fullerene acceptor organic solar cells. Nature Materials 20, 378-384 (2021) (cit. on pp. 18, $35,37)$.

63. Lide, D. R. CRC handbook of chemistry and physics (CRC press, 2004) (cit. on p. 20).

64. Wikipedia. Kirchhoff's law of thermal radiation https://en.wikipedia.org/wiki/ Kirchhoff\%27s_law_of_thermal_radiation\#CITEREFMilne1930 (cit. on p. 22).

65. Rau, U. Reciprocity relation between photovoltaic quantum efficiency and electroluminescent emission of solar cells. Physical Review B 76, 085303 (2007) (cit. on p. 23). 
66. Kirchartz, T. \& Rau, U. Detailed balance and reciprocity in solar cells. physica status solidi (a) 205, 2737-2751 (2008) (cit. on p. 23).

67. Vandewal, K., Tvingstedt, K., Gadisa, A., Inganäs, O. \& Manca, J. V. Relating the open-circuit voltage to interface molecular properties of donor: acceptor bulk heterojunction solar cells. Physical Review B 81, 125204 (2010) (cit. on p. 23).

68. Felekidis, N., Melianas, A. \& Kemerink, M. The role of delocalization and excess energy in the quantum efficiency of organic solar cells and the validity of optical reciprocity relations. The Journal of Physical Chemistry Letters 11, 35633570 (2020) (cit. on p. 23).

69. Ran, N. A. et al. Harvesting the full potential of photons with organic solar cells. Advanced Materials 28, 1482-1488 (2016) (cit. on pp. 25, 29).

70. Cui, Y. et al. Efficient semitransparent organic solar cells with tunable color enabled by an ultralow-bandgap nonfullerene acceptor. Advanced Materials 29, 1703080 (2017) (cit. on p. 25).

71. Firdaus, Y. et al. Long-range exciton diffusion in molecular non-fullerene acceptors. Nature Communications 11, 5220. http://dx. doi .org/10.1038/s41467020-19029-9\%20http: //www . nature . com/articles/s41467-020-19029-9 (Dec. 2020) (cit. on pp. 25, 34).

72. Perdigón-Toro, L. et al. Barrierless free charge generation in the high-performance PM6: Y6 bulk heterojunction non-fullerene solar cell. Advanced Materials 32, 1906763 (2020) (cit. on pp. 25, 37).

73. Wang, J. et al. Organic cavity photodetectors based on nanometer-thick active layers for tunable monochromatic spectral response. ACS Photonics 6, 13931399 (2019) (cit. on p. 25).

74. Kublitski, J. et al. Enhancing sub-bandgap external quantum efficiency by photomultiplication for narrowband organic near-infrared photodetectors. Nature Communications 12, 1-9 (2021) (cit. on p. 25).

75. Yuan, J. et al. Enabling low voltage losses and high photocurrent in fullerenefree organic photovoltaics. Nature communications 10, 1-8(2019) (cit. on pp. 25, 29).

76. Wang, Y. et al. Optical gaps of organic solar cells as a reference for comparing voltage losses. Advanced energy materials 8, 1801352 (2018) (cit. on p. 25).

77. Nikolis, V. C. et al. Reducing voltage losses in cascade organic solar cells while maintaining high external quantum efficiencies. Advanced Energy Materials 7, 1700855 (2017) (cit. on p. 25).

78. Vandewal, K., Benduhn, J. \& Nikolis, V. How to determine optical gaps and voltage losses in organic photovoltaic materials. Sustainable Energy \& Fuels 2, 538544 (2018) (cit. on p. 25).

79. Rau, U., Blank, B., Müller, T. C. \& Kirchartz, T. Efficiency potential of photovoltaic materials and devices unveiled by detailed-balance analysis. Physical review applied 7, 044016 (2017) (cit. on p. 25).

80. Caspar, J. V. \& Meyer, T. J. Application of the energy gap law to nonradiative, excited-state decay. The Journal of Physical Chemistry 87, 952-957 (1983) (cit. on p. 28).

81. Treadway, J. A. et al. Effect of delocalization and rigidity in the acceptor ligand on MLCT excited-state decay. Inorganic chemistry 35, 2242-2246 (1996) (cit. on p. 28).

82. Wei, Y.-C. et al. Overcoming the energy gap law in near-infrared OLEDs by excitonvibration decoupling. Nature Photonics 14, 570-577 (2020) (cit. on p. 28). 
83. Tessler, N., Medvedev, V., Kazes, M., Kan, S. \& Banin, U. Efficient near-infrared polymer nanocrystal light-emitting diodes. Science 295, 1506-1508 (2002) (cit. on p. 28).

84. Benduhn, J. et al. Intrinsic non-radiative voltage losses in fullerene-based organic solar cells. Nature Energy 2, 1-6 (2017) (cit. on pp. 28, 29).

85. Ullbrich, S. et al. Emissive and charge-generating donor-acceptor interfaces for organic optoelectronics with low voltage losses. Nature Materials 18, 459464 (2019) (cit. on p. 28).

86. Gadisa, A., Svensson, M., Andersson, M. R. \& Inganäs, O. Correlation between oxidation potential and open-circuit voltage of composite solar cells based on blends of polythiophenes/fullerene derivative. Applied Physics Letters 84, 1609_ 1611 (2004) (cit. on p. 29).

87. Scharber, M. C. et al. Design rules for donors in bulk-heterojunction solar cellsTowards 10\% energy-conversion efficiency. Advanced materials 18, 789-794 (2006) (cit. on p. 29).

88. Ross, R. B. et al. Endohedral fullerenes for organic photovoltaic devices. Nature materials 8, 208-212 (2009) (cit. on p. 29).

89. Lenes, M. et al. Fullerene bisadducts for enhanced open-circuit voltages and efficiencies in polymer solar cells. Advanced Materials 20, 2116-2119 (2008) (cit. on p. 29).

90. Zhang, F. et al. High photovoltage achieved in low band gap polymer solar cells by adjusting energy levels of a polymer with the LUMOs of fullerene derivatives. Journal of Materials Chemistry 18, 5468-5474 (2008) (cit. on p. 29).

91. Chen, X.-K. et al. A unified description of non-radiative voltage losses in organic solar cells. Nature Energy, 1-8 (2021) (cit. on pp. 29-31).

92. Eisner, F. D. et al. Hybridization of local exciton and charge-transfer states reduces nonradiative voltage losses in organic solar cells. Journal of the American Chemical Society 141, 6362-6374 (2019) (cit. on p. 29).

93. Yang, C. et al. Effects of energy-level offset between a donor and acceptor on the photovoltaic performance of non-fullerene organic solar cells. Journal of Materials Chemistry A 7, 18889-18897 (2019) (cit. on p. 29).

94. Cai, Y. et al. Effect of the Energy Offset on the Charge Dynamics in Nonfullerene Organic Solar Cells. ACS Applied Materials \& Interfaces 12, 43984-43991 (2020) (cit. on p. 29).

95. Liu, S. et al. High-efficiency organic solar cells with low non-radiative recombination loss and low energetic disorder. Nature Photonics 14, 300-305 (2020) (cit. on pp. 30, 34).

96. Hou, J., Inganäs, O., Friend, R. H. \& Gao, F. Organic solar cells based on nonfullerene acceptors. Nature Materials 17, 119-128 (2018) (cit. on pp. 31, 34).

97. Perdigón-Toro, L. et al. Excitons dominate the emission from PM6: Y6 solar cells, but this does not help the open-circuit voltage of the device. ACS Energy Letters 6, 557-564 (2021) (cit. on p. 31).

98. Dai, S. et al. Fused nonacyclic electron acceptors for efficient polymer solar cells. Journal of the American Chemical Society 139, 1336-1343 (2017) (cit. on p. 34).

99. Haugeneder, A. et al. Exciton diffusion and dissociation in conjugated polymer/fullerene blends and heterostructures. Physical Review B 59, 15346 (1999) (cit. on p. 34). 
100. Markov, D. E., Amsterdam, E., Blom, P. W., Sieval, A. B. \& Hummelen, J. C. Accurate measurement of the exciton diffusion length in a conjugated polymer using a heterostructure with a side-chain cross-linked fullerene layer. The Journal of Physical Chemistry A 109, 5266-5274 (2005) (cit. on p. 34).

101. Scully, S. R. \& McGehee, M. D. Effects of optical interference and energy transfer on exciton diffusion length measurements in organic semiconductors. Journal of Applied Physics 100, 034907 (2006) (cit. on p. 34).

102. Mikhnenko, O. et al. Temperature dependence of exciton diffusion in conjugated polymers. The Journal of Physical Chemistry B 112, 11601-11604 (2008) (cit. on p. 34).

103. Mikhnenko, O. V. et al. Exciton diffusion length in narrow bandgap polymers. Energy \& Environmental Science 5, 6960-6965 (2012) (cit. on p. 34).

104. Marcus, R. A. On the theory of oxidation-reduction reactions involving electron transfer. I. The Journal of chemical physics 24, 966-978 (1956) (cit. on p. 35).

105. Jortner, J. Temperature dependent activation energy for electron transfer between biological molecules. The Journal of Chemical Physics 64, 4860-4867 (1976) (cit. on p. 35).

106. Chen, K., Barker, A. J., Reish, M. E., Gordon, K. C. \& Hodgkiss, J. M. Broadband ultrafast photoluminescence spectroscopy resolves charge photogeneration via delocalized hot excitons in polymer: fullerene photovoltaic blends. Journal of the American Chemical Society 135, 18502-18512 (2013) (cit. on p. 35).

107. Falke, S. M. et al. Coherent ultrafast charge transfer in an organic photovoltaic blend. Science 344, 1001-1005 (2014) (cit. on p. 35).

108. Eastham, N. D. et al. Hole-transfer dependence on blend morphology and energy level alignment in polymer: ITIC photovoltaic materials. Advanced materials 30, 1704263 (2018) (cit. on p. 35).

109. Li, S. et al. Highly efficient fullerene-free organic solar cells operate at near zero highest occupied molecular orbital offsets. Journal of the American Chemical Society 141, 3073-3082 (2019) (cit. on p. 35).

110. Gélinas, S. et al. The binding energy of charge-transfer excitons localized at polymeric semiconductor heterojunctions. The Journal of Physical Chemistry $C$ 1 15, 7114-7119 (2011) (cit. on p. 36).

111. Burke, T. M., Sweetnam, S., Vandewal, K. \& McGehee, M. D. Beyond Langevin recombination: How equilibrium between free carriers and charge transfer states determines the open-circuit voltage of organic solar cells. Advanced Energy Materials 5, 1500123 (2015) (cit. on pp. 36, 40).

112. Menke, S. M. et al. Order enables efficient electron-hole separation at an organic heterojunction with a small energy loss. Nature communications $\mathbf{9}, 1-7$ (2018) (cit. on p. 36).

113. Vandewal, K. et al. Efficient charge generation by relaxed charge-transfer states at organic interfaces. Nature materials 13, 63-68 (2014) (cit. on p. 37).

114. Veldman, D. et al. Compositional and electric field dependence of the dissociation of charge transfer excitons in alternating polyfluorene copolymer/fullerene blends. Journal of the American Chemical Society 130, 7721-7735 (2008) (cit. on p. 37).

115. Bässler, H. \& Köhler, A. "Hot or cold": how do charge transfer states at the donoracceptor interface of an organic solar cell dissociate? Physical Chemistry Chemical Physics 17, 28451-28462 (2015) (cit. on p. 37). 
116. Savoie, B. M., Jackson, N. E., Chen, L. X., Marks, T. J. \& Ratner, M. A. Mesoscopic features of charge generation in organic semiconductors. Accounts of Chemical Research 47, 3385-3394 (2014) (cit. on p. 37).

117. Gélinas, S. et al. Ultrafast long-range charge separation in organic semiconductor photovoltaic diodes. Science 343, 512-516 (2014) (cit. on p. 37).

118. Nan, G., Zhang, X. \& Lu, G. Do "Hot" Charge-Transfer Excitons Promote Free Carrier Generation in Organic Photovoltaics? The Journal of Physical Chemistry C 119, 15028-15035 (2015) (cit. on p. 37).

119. Huix-Rotllant, M., Tamura, H. \& Burghardt, I. Concurrent effects of delocalization and internal conversion tune charge separation at regioregular polythiophenefullerene heterojunctions. The Journal of Physical Chemistry Letters 6, 17021708 (2015) (cit. on p. 37).

120. Monahan, N. R., Williams, K. W., Kumar, B., Nuckolls, C. \& Zhu, X.-Y. Direct observation of entropy-driven electron-hole pair separation at an organic semiconductor interface. Physical Review Letters 1 14, 247003 (2015) (cit. on p. 37).

121. Gao, F., Tress, W., Wang, J. \& Inganäs, O. Temperature dependence of charge carrier generation in organic photovoltaics. Physical Review Letters 114, 128701 (2015) (cit. on p. 37).

122. Yu, Z., Smith, D., Saxena, A., Martin, R. \& Bishop, A. Molecular geometry fluctuations and field-dependent mobility in conjugated polymers. Physical Review B 63, 085202 (2001) (cit. on p. 37).

123. Bouhassoune, M., Van Mensfoort, S., Bobbert, P. \& Coehoorn, R. Carrier-density and field-dependent charge-carrier mobility in organic semiconductors with correlated Gaussian disorder. Organic Electronics 10, 437-445 (2009) (cit. on p. 37).

124. Bange, S., Schubert, M. \& Neher, D. Charge mobility determination by current extraction under linear increasing voltages: Case of nonequilibrium charges and field-dependent mobilities. Physical Review B 81, 035209 (2010) (cit. on p. 37).

125. Baranovski, S. Charge transport in disordered solids with applications in electronics (John Wiley \& Sons, 2006) (cit. on p. 37).

126. Silbey, R. \& Munn, R. General theory of electronic transport in molecular crystals. i. local linear electron-phonon coupling. The Journal of Chemical Physics 72, 2763-2773 (1980) (cit. on p. 37).

127. Sewell, G. Model of thermally activated hopping motion in solids. Physical Review 129, 597 (1963) (cit. on p. 37).

128. Emin, D. Thermoelectric power due to electronic hopping motion. Physical Review Letters 35, 882 (1975) (cit. on p. 37).

129. Bässler, H. Charge transport in disordered organic photoconductors. A Monte Carlo simulation study. Physica Status Solidi B (Basic Research);(Germany) 175 (1993) (cit. on p. 38).

130. Miller, A. \& Abrahams, E. Impurity conduction at low concentrations. Physical Review 120, 745 (1960) (cit. on p. 38).

131. Schnönherr, G., Eiermann, R., Bässler, H. \& Silver, M. Dispersive exciton transport in a hopping system with gaussian energy distribution. Chemical Physics 52, 287-298 (1980) (cit. on p. 38).

132. Offermans, T., van Hal, P. A., Meskers, S. C., Koetse, M. M. \& Janssen, R. A. Exciplex dynamics in a blend of $\pi$-conjugated polymers with electron donating and accepting properties: MDMO-PPV and PCNEPV. Physical Review B 72, 045213 (2005) (cit. on p. 38). 
133. Pasveer, W. et al. Unified description of charge-carrier mobilities in disordered semiconducting polymers. Physical Review Letters 94, 206601 (2005) (cit. on p. 38).

134. Houili, H., Tutiš, E., Batistić, I. \& Zuppiroli, L. Investigation of the charge transport through disordered organic molecular heterojunctions. Journal of Applied Physics 100, 033702 (2006) (cit. on p. 38).

135. McNaught, A. D., Wilkinson, A., et al. Compendium of chemical terminology (Blackwell Science Oxford, 1997) (cit. on p. 39).

136. Pope, M., Swenberg, C. E., et al. Electronic processes in organic crystals and polymers (Oxford University Press on Demand, 1999) (cit. on p. 40).

137. Langevin, P. Recombinaison et mobilites des ions dans les gaz. Annales de Chimie et de Physique 28, 122 (1903) (cit. on p. 40).

138. Deibel, C., Baumann, A. \& Dyakonov, V. Polaron recombination in pristine and annealed bulk heterojunction solar cells. Applied Physics Letters 93, 386 (2008) (cit. on p. 40).

139. Juška, G., Arlauskas, K., Stuchlik, J. \& Österbacka, R. Non-Langevin bimolecular recombination in low-mobility materials. Journal of Non-Crystalline Solids 352, 1167-1171 (2006) (cit. on p. 40).

140. Adriaenssens, G. \& Arkhipov, V. Non-Langevin recombination in disordered materials with random potential distributions. Solid State Communications 103, 541-543 (1997) (cit. on p. 40).

141. Kirchartz, T., Pieters, B. E., Kirkpatrick, J., Rau, U. \& Nelson, J. Recombination via tail states in polythiophene: fullerene solar cells. Physical Review B 83, 115209 (2011) (cit. on p. 40).

142. Kniepert, J., Lange, I., Van Der Kaap, N. J., Koster, L. J. A. \& Neher, D. A conclusive view on charge generation, recombination, and extraction in as-prepared and annealed P3HT: PCBM blends: combined experimental and simulation work. Advanced Energy Materials 4, 1301401 (2014) (cit. on p. 40).

143. Deibel, C., Wagenpfahl, A. \& Dyakonov, V. Origin of reduced polaron recombination in organic semiconductor devices. Physical Review B 80, 075203 (2009) (cit. on p. 40).

144. Armin, A. et al. Reduced recombination in high efficiency molecular nematic liquid crystalline: fullerene solar cells. Advanced Energy Materials 6, 1600939 (2016) (cit. on p. 40).

145. Hosseini, S. M. et al. Putting order into PM6:Y6 solar cells to reduce the Langevin recombination in $400 \mathrm{~nm}$ thick junction. Solar RRL 4, 2000498 (2020) (cit. on p. 40).

146. Kurpiers, J. Probing the pathways of free charge generation and recombination in organic solar cells: the role of excess energy and dispersive effects $\mathrm{PhD}$ thesis (Universität Potsdam, 2019) (cit. on pp. 41, 59).

147. Shuttle, C. et al. Experimental determination of the rate law for charge carrier decay in a polythiophene: Fullerene solar cell. Applied Physics Letters 92, 80 (2008) (cit. on p. 41).

148. Gorenflot, J. et al. From recombination dynamics to device performance: quantifying the efficiency of exciton dissociation, charge separation, and extraction in bulk heterojunction solar cells with fluorine-substituted polymer donors. Advanced Energy Materials 8, 1701678 (2018) (cit. on p. 41).

149. Green, M. A. Accuracy of analytical expressions for solar cell fill factors. Solar Cells 7, 337-340 (1982) (cit. on p. 42). 
150. Neher, D., Kniepert, J., Elimelech, A. \& Koster, L. J. A. A new figure of merit for organic solar cells with transport-limited photocurrents. Scientific Reports 6, 1-9 (2016) (cit. on pp. 42-45).

151. Würfel, U., Neher, D., Spies, A. \& Albrecht, S. Impact of charge transport on current-voltage characteristics and power-conversion efficiency of organic solar cells. Nature Communications 6, 1-9 (2015) (cit. on p. 42).

152. Bartesaghi, D. et al. Competition between recombination and extraction of free charges determines the fill factor of organic solar cells. Nature Communications 6, 1-10 (2015) (cit. on p. 43).

153. Kaienburg, P., Rau, U. \& Kirchartz, T. Extracting information about the electronic quality of organic solar-cell absorbers from fill factor and thickness. Physical Review Applied 6, 024001 (2016) (cit. on p. 44).

154. Armin, A. et al. A history and perspective of non-fullerene electron acceptors for organic solar cells. Advanced Energy Materials 11, 2003570 (2021) (cit. on p. 45).

155. Kirchartz, T., Agostinelli, T., Campoy-Quiles, M., Gong, W. \& Nelson, J. Understanding the thickness-dependent performance of organic bulk heterojunction solar cells: the influence of mobility, lifetime, and space charge. The Journal of Physical Chemistry Letters 3, 3470-3475 (2012) (cit. on p. 45).

156. Tress, W. et al. Correlation of absorption profile and fill factor in organic solar cells: the role of mobility imbalance. Advanced Energy Materials 3, 631-638 (2013) (cit. on p. 45).

157. Stolterfoht, M., Armin, A., Philippa, B. \& Neher, D. The role of space charge effects on the competition between recombination and extraction in solar cells with low-mobility photoactive layers. The Journal of Physical Chemistry Letters 7, 4716-4721 (2016) (cit. on p. 45).

158. Yuan, J. et al. Understanding energetic disorder in electron-deficient-core-based non-fullerene solar cells. Science China Chemistry 63, 1159-1168 (2020) (cit. on p. 45).

159. Guo, X. et al. Polymer solar cells with enhanced fill factors. Nature Photonics 7, 825-833 (2013) (cit. on p. 46).

160. Shaheen, S. E. et al. 2.5\% efficient organic plastic solar cells. Applied Physics Letters 78, 841-843 (2001) (cit. on p. 46).

161. Li, G. et al. High-efficiency solution processable polymer photovoltaic cells by self-organization of polymer blends. Nature Materials 4 (2005) (cit. on p. 46).

162. Vohra, V. et al. Efficient inverted polymer solar cells employing favourable molecular orientation. Nature Photonics 9, 403-408 (2015) (cit. on p. 46).

163. He, Z. et al. Single-junction polymer solar cells with high efficiency and photovoltage. Nature Photonics 9, 174-179 (2015) (cit. on p. 46).

164. Sun, K. et al. A molecular nematic liquid crystalline material for high-performance organic photovoltaics. Nature Communications 6, 1-9 (2015) (cit. on p. 46).

165. Zhao, W. et al. Fullerene-free polymer solar cells with over $11 \%$ efficiency and excellent thermal stability. Advanced Materials 28, 4734-4739 (2016) (cit. on p. 46).

166. Yuan, J. et al. Single-junction organic solar cell with over $15 \%$ efficiency using fused-ring acceptor with electron-deficient core. Joule 3, 1140-1151 (2019) (cit. on p. 46). 
167. Bi, P. et al. Reduced non-radiative charge recombination enables organic photovoltaic cell approaching $19 \%$ efficiency. Joule (2021) (cit. on p. 46).

168. Zhang, X. et al. On the understanding of energy loss and device fill factor tradeoffs in non-fullerene organic solar cells with varied energy levels. Nano Energy 75, 105032 (2020) (cit. on p. 46).

169. Sun, R. et al. Achieving eco-compatible organic solar cells with efficiency $>16.5 \%$ based on an Iridium complex-incorporated polymer donor. Solar RRL 4, 2000156 (2020) (cit. on p. 50).

170. FeuRenard. Wikimedia commons: Diagram of a Czerny-Turner monochromator https : //commons.wikimedia.org/wiki/File:Czerny-Turner_Monochromator.svg (cit. on p. 52). 



\section{Papers}

The papers associated with this thesis have been removed for copyright reasons. For more details about these see:

http://urn.kb.se/resolve?urn=urn:nbn:se:liu:diva-178408 


\section{FACULTY OF SCIENCE AND ENGINEERING}

Linköping Studies in Science and Technology, Dissertation No. 2173, 2021

Department of Physics, Chemistry and Biology (IFM)

Linköping University

SE-581 83 Linköping, Sweden

www.liu.se 